\title{
Tight Quotients and Double Quotients in the Bruhat Order
}

\author{
John R. Stembridge* \\ Department of Mathematics \\ University of Michigan \\ Ann Arbor, Michigan 48109-1109 USA \\ jrs@umich.edu \\ Dedicated to Richard Stanley on the occasion of his 60th birthday \\ Submitted: Aug 17, 2004; Accepted: Jan 31, 2005; Published: Feb 14, 2005 \\ Mathematics Subject Classifications: 06A07, 20F55
}

\begin{abstract}
It is a well-known theorem of Deodhar that the Bruhat ordering of a Coxeter group is the conjunction of its projections onto quotients by maximal parabolic subgroups. Similarly, the Bruhat order is also the conjunction of a larger number of simpler quotients obtained by projecting onto two-sided (i.e., "double") quotients by pairs of maximal parabolic subgroups. Each one-sided quotient may be represented as an orbit in the reflection representation, and each double quotient corresponds to the portion of an orbit on the positive side of certain hyperplanes. In some cases, these orbit representations are "tight" in the sense that the root system induces an ordering on the orbit that yields effective coordinates for the Bruhat order, and hence also provides upper bounds for the order dimension. In this paper, we (1) provide a general characterization of tightness for one-sided quotients, (2) classify all tight one-sided quotients of finite Coxeter groups, and (3) classify all tight double quotients of affine Weyl groups.
\end{abstract}

\section{Introduction.}

The Bruhat orderings of Coxeter groups and their parabolic quotients have a long history that originates with the fact that these posets (in the case of finite Weyl groups) record the inclusion of cell closures in generalized flag varieties.

Some of the significant early papers on the combinatorial aspects of this subject include the 1977 paper of Deodhar [D1] providing various characterizations of the Bruhat order (including some that will be essential in this work), the 1980 paper of Stanley [St] in which Bruhat orderings of finite Weyl groups and their parabolic quotients are shown to be strongly Sperner, and the 1982 paper of Björner and Wachs in which the Bruhat order is shown to be lexicographically shellable $[\mathbf{B W}]$.

\footnotetext{
* This work was supported by NSF grant DMS-0245385.
} 
In this paper, we investigate the explicit assignment of coordinates for the Bruhat order. By a "coordinate assignment" for a poset $P$, we mean an order-embedding $P \rightarrow \mathbf{R}^{d}$; i.e., an injective map $f: P \rightarrow \mathbf{R}^{d}$ such that $x<y$ in $P$ if and only if $f(x)<f(y)$ in the usual (coordinate-wise) partial ordering of $\mathbf{R}^{d}$. The minimum such $d$ for which this is possible is known as the order dimension of $P$, and denoted $\operatorname{dim} P$.

For example, Proctor $[\mathbf{P 1}]$ has given coordinates for the Bruhat orderings of the classical finite Coxeter groups and their quotients, and more recently, Reading $[\mathbf{R}]$ has determined the exact order dimensions of the Bruhat orderings of $A_{n}, B_{n}, H_{3}$, and $H_{4}$. It would be interesting to have a uniform construction of coordinates for the Bruhat orders of finite Weyl groups, perhaps based directly on the geometry of flag varieties as in Proposition 7.1 of $[\mathbf{P 1}$ ] for type $A$. For the infinite Coxeter groups, perhaps the most interesting question is the classification of those groups for which the Bruhat ordering is finite-dimensional. Indeed, Reading and Waugh $[\mathbf{R W}]$ have shown that there are Coxeter groups whose Bruhat order has infinite order dimension, and infinite Coxeter groups (such as the affine Weyl groups of type $A$ ) with finite order dimension.

Our initial motivation for this work began with the observation that for each finite Weyl group $W$ and associated affine Weyl group $\widetilde{W}$, the two-sided (parabolic) quotient $W \backslash \widetilde{W} / W$ may be naturally identified with the dominant part of the co-root lattice. We were surprised to realize that the Bruhat ordering of $W \backslash \widetilde{W} / W$ is isomorphic to the usual ordering of dominant co-weights: moving up in this Bruhat order is equivalent to adding positive combinations of positive co-roots. (Later, we learned from M. Dyer that this is mentioned explicitly in Section 2 of $[\mathbf{L}]$.) This meant that the various remarkable properties of the partial order of dominant (co-)weights (see for example [S2]) could be transfered to the Bruhat ordering of certain two-sided quotients of affine Weyl groups.

At this point, we began to investigate more general instances of this phenomenon. Indeed, it is always possible to identify a one-sided parabolic quotient of any Coxeter group with the orbit of a point in the reflection representation, and a two-sided (or "double") quotient corresponds to the part of an orbit on the positive side of certain hyperplanes. In these terms, a necessary condition for moving up in the Bruhat order requires adding (or subtracting, depending on conventions) positive combinations of positive roots. The interesting question is one of identifying when this necessary condition is sufficient. That is, when do the root coordinates of an orbit, or the portion corresponding to some double quotient, provide an order embedding of the corresponding Bruhat order? The main goal of this paper is to identify these "tight" quotients.

An outline of the paper follows.

In Section 1, we discuss the details of using the reflection representation of a Coxeter group to model the Bruhat orderings of its parabolic quotients. We also review a key result of Deodhar (see Theorem 1.3) that allows the Bruhat ordering of $W$ to be recovered from its projections onto one-sided or two-sided quotients.

In Section 2, we formalize the notion of a tight quotient, and prove a purely ordertheoretic characterization of the tight one-sided quotients (Theorem 2.3): the Bruhat ordering of $W / W_{J}$ is tight if and only if the Bruhat ordering of $W_{I} \backslash W / W_{J}$ is a chain for every maximal parabolic subgroup $W_{I}$ of $W$. We also point out that the Bruhat 
orderings of minuscule (one-sided) quotients are always tight.

In Section 3, we classify the tight one-sided quotients of finite Coxeter groups. We expected the results to include only a few instances beyond the minuscule cases (a frequent outcome in the theory of finite Coxeter groups), but were instead surprised to discover that there are many other examples, including quotients by non-maximal parabolic subgroups.

In the course of deriving the classification, we develop two significant necessary conditions for tightness. The first involves the "stratification" of an orbit relative to the action of a parabolic subgroup, and the second involves confining a face of the dominant chamber inside a face of the "double weight arrangement" of hyperplanes (an arrangement that is in general much larger than the usual arrangement defined by the root hyperplanes). In fact, both of these necessary conditions may be used to provide characterizations of tightness (see Lemma 3.3, Theorem 3.9, and Corollary 3.10), although our proofs of the latter two depend a posteriori on the classification.

In the final two sections, we focus on the affine Weyl groups. For these groups, there are two natural representations: the first is the usual reflection representationavailable for all Coxeter groups - in which the group is represented via linear operators; in the second, one uses affine transformations. In Section 4, we present a dictionary for translating between these two points of view, and prove that there are no one-sided or double quotients that are tight relative to the reflection representation, apart from some trivial cases (Theorem 4.9). In contrast, we show that double quotients with both factors of minuscule type are tight relative to the affine representation (Theorem 4.10).

In Section 5, we turn to the classification of quotients of affine Weyl groups that are tight relative to the affine representation. In particular, Theorem 5.12 and Corollary 5.13 provide a classification of all double quotients with a tight embedding in some affine orbit; we find that the left factor must be of minuscule type, but there is a larger number of possibilities for the right factor. The proof has a structure similar to the one in Section 3-we find that there are affine analogues of orbit stratification and the double weight arrangement that provide characterizations of tightness similar to those we develop for finite Coxeter groups (see Theorems 5.10 and 5.11).

\section{Acknowledgment.}

I would like to thank Nathan Reading for many helpful discussions.

\section{The Bruhat order.}

Let $(W, S)$ be a Coxeter system. Via the reflection representation, one may view $W$ as a group of isometries of some real vector space $V$ equipped with a (not necessarily positive definite) inner product $\langle$,$\rangle . In particular, we may associate with W$ a centrallysymmetric, $W$-invariant subset $\Phi \subset V-\{0\}$ (the root system) so that the reflections in $W$ are the linear transformations $s_{\beta}: \lambda \mapsto \lambda-\langle\lambda, \beta\rangle \beta^{\vee}$, where $\beta$ varies over $\Phi$, and $\beta^{\vee}:=2 \beta /\langle\beta, \beta\rangle$ denotes the co-root corresponding to $\beta .{ }^{1}$ In this framework, the

\footnotetext{
${ }^{1}$ For the details of this construction, we refer the reader to (for example) Chapter 5 of $[\mathbf{H}]$, although it should be noted that the normalization $\langle\beta, \beta\rangle=1$ for $\beta \in \Phi$ in $[\mathbf{H}]$ may be relaxed-rescaling each $W$-orbit of roots by an arbitrary positive scalar has no significant effect on the general theory.
} 
generating set $S$ is the set of simple reflections: for each $s \in S$ one may choose a root $\alpha$ (designated to be simple) so that $s=s_{\alpha}$, and these choices may be arranged so that every root is in either the nonnegative or nonpositive span of the simple roots. Thus $\Phi$ is the disjoint union of $\Phi^{+}$(the positive roots) and $\Phi^{-}=-\Phi^{+}$(the negative roots).

For $w \in W$, let $\ell(w)$ denote the minimum length of an expression $w=s_{1} \cdots s_{l}$ $\left(s_{i} \in S\right)$. A key relationship between the root system and length is the fact that

$$
\ell(w)<\ell\left(s_{\beta} w\right) \Leftrightarrow w^{-1} \beta \in \Phi^{+} \quad\left(w \in W, \beta \in \Phi^{+}\right),
$$

and the Bruhat ordering of $W$ may be defined as the transitive closure of the relations

$$
w<_{\mathrm{B}} s_{\beta} w
$$

for all $w \in W$ and $\beta \in \Phi^{+}$satisfying either of the equivalent conditions in (1.1).

For each $J \subset S$, we let $W_{J}$ denote the parabolic subgroup of $W$ generated by $J$, and $\Phi_{J} \subset \Phi$ the corresponding root subsystem. One knows that

$$
\begin{aligned}
& W^{J}:=\{w \in W: \ell(w s)>\ell(w) \text { for all } s \in J\} \\
& { }^{J} W:=\{w \in W: \ell(s w)>\ell(w) \text { for all } s \in J\}
\end{aligned}
$$

are the unique sets of coset representatives for $W / W_{J}$ and $W_{J} \backslash W$ (respectively) that minimize length, and similarly (Exercise IV.1.3 of $[\mathbf{B}]$ )

$$
{ }^{I} W^{J}:={ }^{I} W \cap W^{J}
$$

is the unique set of length-minimizing representatives for the double cosets $W_{I} \backslash W / W_{J}$.

\section{A. Orbits and one-sided quotients.}

If $\theta \in V$ is dominant (i.e., $\langle\theta, \beta\rangle \geqslant 0$ for all $\beta \in \Phi^{+}$), then the $W$-stabilizer of $\theta$ is the parabolic subgroup $W_{J}$, where $J=\left\{s_{\alpha} \in S:\langle\theta, \alpha\rangle=0\right\}$. This allows $W / W_{J}$ to be identified with the $W$-orbit of $\theta$, and as previously noted in [S4], the following result shows that the poset structure of $W^{J}$ (as a subposet of $\left(W,<_{\mathrm{B}}\right)$ ) may be transported to a partial ordering on $W \theta$ by taking the transitive closure of the relations

$$
\mu<_{\mathrm{B}} s_{\beta}(\mu) \quad \text { for all } \beta \in \Phi^{+} \text {such that }\langle\mu, \beta\rangle>0 \text {. }
$$

Proposition $1.1[\mathbf{S 4}]$. Assume $\theta \in V$ is dominant with stabilizer $W_{J}$.

(a) Evaluation (i.e., $w \mapsto w \theta)$ is an order-preserving map $\left(W,<_{\mathrm{B}}\right) \rightarrow\left(W \theta,<_{\mathrm{B}}\right)$.

(b) The evaluation map restricts to a poset isomorphism $\left(W^{J},<_{\mathrm{B}}\right) \rightarrow\left(W \theta,<_{\mathrm{B}}\right)$.

Proof. (a) If $w<_{\mathrm{B}} s_{\beta} w$ is a covering relation in $\left(W,<_{\mathrm{B}}\right)$, then (1.1) implies that $w^{-1} \beta$ is a positive root, so $\langle w \theta, \beta\rangle=\left\langle\theta, w^{-1} \beta\right\rangle \geqslant 0$. Hence either $w \theta=s_{\beta} w \theta$ (if $\langle w \theta, \beta\rangle=0$ ) or $w \theta<_{\mathrm{B}} s_{\beta} w \theta$ (if $\langle w \theta, \beta\rangle>0$ ), so $w \theta \leqslant_{\mathrm{B}} s_{\beta} w \theta$ in both cases. 
(b) Since $W_{J}$ is the stabilizer of $\theta$, it is clear that the evaluation map is a bijection between $W^{J}$ and $W \theta$, so we need only to show that the inverse map is order-preserving. Thus suppose we have a covering relation $\mu<_{\mathrm{B}} s_{\beta}(\mu)$ in $\left(W \theta,<_{\mathrm{B}}\right)$ for some $\operatorname{root} \beta \in \Phi^{+}$. We necessarily have $\langle\mu, \beta\rangle>0$, so if $w$ is the unique member of $W^{J}$ such that $\mu=w \theta$, then $\left\langle\theta, w^{-1} \beta\right\rangle=\langle\mu, \beta\rangle>0$, so $w^{-1} \beta$ is a positive root and $w<_{\mathrm{B}} s_{\beta} w$.

Now let $x \in W_{J}$ be the unique element such that $s_{\beta} w x \in W^{J}$. It follows easily from the definition that each member of $W^{J}$ is the Bruhat-minimum of its coset, so $w \leqslant_{\mathrm{B}} w x$. Furthermore, it is clear that $s_{\beta} w x$ and $w x$ must be related in Bruhat order. However, $s_{\beta} w x<_{\mathrm{B}} w x$ would contradict (a), so in fact $w \leqslant_{\mathrm{B}} w x<_{\mathrm{B}} s_{\beta} w x$ and the result follows.

REMARK 1.2. (a) One complication for infinite Coxeter groups is that the bilinear form $\langle$,$\rangle may be degenerate on V$. However, it is always possible to replace $V$ with a larger space and extend the bilinear form in a non-degenerate way. This allows us to identify $V$ with its dual space, and guarantee that for every parabolic subgroup $W_{J}$, there is a dominant point in $V$ whose stabilizer is $W_{J}$.

(b) (Proposition 3 of $[\mathbf{P} 1]$ ). The quantity $\langle\mu+t \beta, \mu+t \beta\rangle$ is a quadratic function of $t$, and $\mu \mapsto\langle\mu, \mu\rangle$ is constant on $W$-orbits, so for each root $\beta$ there is at most one other point in the $W$-orbit of $\mu$ of the form $\mu+t \beta$ (namely, $s_{\beta}(\mu)$ ). It follows that the Bruhat ordering of $W \theta$ may alternatively be defined as the transitive closure of all relations $\mu<\nu(\mu, \nu \in W \theta)$ such that $\mu-\nu$ is a positive multiple of a positive root.

(c) One knows that the Bruhat ordering of $W^{J}$ is graded by length (e.g., see [D1]). If we transport this to $\left(W \theta,<_{\mathrm{B}}\right)$, we obtain the rank function

$$
r(\mu):=\left|\left\{\beta \in \Phi^{+}:\langle\mu, \beta\rangle<0\right\}\right| \quad(\mu \in W \theta) .
$$

Indeed, given $\mu=w \theta$ and $w \in W^{J}$, there are three possibilities for each $\beta \in \Phi^{+}$, depending on the sign of $\langle\mu, \beta\rangle=\left\langle\theta, w^{-1} \beta\right\rangle$ : if it is negative, then $w^{-1} \beta \in \Phi^{-}$; if it is positive, then $w^{-1} \beta \in \Phi^{+}$; if it vanishes, then $w^{-1} \beta \in \Phi_{J}$, and hence $w^{-1} \beta \in \Phi^{+}$ (otherwise, we contradict (1.1) and the fact that $w \in W^{J}$ ). Hence $r(\mu)=\left|\Phi^{+} \cap w \Phi^{-}\right|$, a well-known expression for the length of $w$ (e.g., see Section 5.6 of $[\mathbf{H}]$ ).

Let $\pi_{J}: W \rightarrow W^{J}$ denote the natural projection map (i.e., $\pi_{J}(x y)=x$ for all $x \in W^{J}$ and $y \in W_{J}$ ). An immediate corollary of Proposition 1.1 is the well-known fact that $\pi_{J}$ is order-preserving. As a sort of converse to this, we have

Theorem 1.3 (Deodhar [D1]). For all $I, J \subseteq S$ and $x, y \in W$, we have

$$
\pi_{I \cap J}(x) \leqslant_{\mathrm{B}} \pi_{I \cap J}(y) \text { if and only if } \pi_{I}(x) \leqslant_{\mathrm{B}} \pi_{I}(y) \text { and } \pi_{J}(x) \leqslant_{\mathrm{B}} \pi_{J}(y) \text {. }
$$

It will be convenient for what follows to use the abbreviation $\langle s\rangle$ for $S-\{s\}$.

Corollary 1.4. For all $J \subseteq S$ and all $x, y \in W^{J}$, we have $x \leqslant_{\mathrm{B}} y$ if and only if $\pi_{\langle s\rangle}(x) \leqslant_{\mathrm{B}} \pi_{\langle s\rangle}(y)$ for all $s \in S-J$. 
It follows that an assignment of coordinates for the Bruhat ordering of any parabolic quotient $W^{J}$ (including the full group $W$ in the case $J=\varnothing$ ) may be produced once we have coordinates for the quotients by maximal parabolic subgroups. In particular, we may deduce bounds on the order dimension; viz.,

$$
\operatorname{dim}\left(W^{J},<_{\mathrm{B}}\right) \leqslant \sum_{s \in S-J} \operatorname{dim}\left(W^{\langle s\rangle},<_{\mathrm{B}}\right)
$$

\section{B. Double quotients.}

Given $I \subseteq S$ and a dominant $\theta \in V$ with stabilizer $W_{J}$, let

$$
(W \theta)_{I}:=\left\{\mu \in W \theta:\langle\mu, \alpha\rangle \geqslant 0 \text { for all } \alpha \in \Phi_{I}^{+}\right\}
$$

denote the subset of $W \theta$ that is dominant with respect to $\Phi_{I}$. The following result shows that the subposet of $\left(W \theta,<_{\mathrm{B}}\right)$ formed by $(W \theta)_{I}$ is isomorphic to the Bruhat ordering of the double quotient ${ }^{I} W^{J}$, and that this subposet may be generated by the application of certain reflections.

Proposition 1.5. Assume $\theta \in V$ is dominant with stabilizer $W_{J}$.

(a) If $w \in{ }^{I} W$, then $w \theta \in(W \theta)_{I}$.

(b) If $w \in W^{J}$ and $w \theta \in(W \theta)_{I}$, then $w \in{ }^{I} W^{J}$. (Hence, the evaluation map $w \mapsto w \theta$ restricts to a bijection between ${ }^{I} W^{J}$ and $(W \theta)_{I}$.)

(c) The partial ordering of $(W \theta)_{I}$, as a subposet of $\left(W \theta,<_{\mathrm{B}}\right)$, is generated by the transitive closure of the relations $\mu<_{\mathrm{B}} s_{\beta}(\mu)$ for all $\mu \in(W \theta)_{I}$ and $\beta \in \Phi^{+}$ such that $s_{\beta}(\mu) \in(W \theta)_{I}$ and $\langle\mu, \beta\rangle>0$.

Proof. (a) If $w \in{ }^{I} W$, then we have $w^{-1} \alpha \in \Phi^{+}$for all simple $\alpha \in \Phi_{I}$, and hence also for all $\alpha \in \Phi_{I}^{+}$. It follows that $\langle w \theta, \alpha\rangle=\left\langle\theta, w^{-1} \alpha\right\rangle \geqslant 0$ for all such $\alpha$; i.e., $w \theta \in(W \theta)_{I}$.

(b) Suppose $w \in W^{J}$ and $w \theta \in(W \theta)_{I}$. If $w$ failed to be in ${ }^{I} W$, then we would have $\ell\left(s_{\alpha} w\right)<\ell(w)$ for some simple $\alpha \in \Phi_{I}$, whence $w^{-1} \alpha \in \Phi^{-}$and $\langle w \theta, \alpha\rangle \leqslant 0$. However, given that $w \theta$ is $\Phi_{I^{-}}$dominant, this is possible only if $\langle w \theta, \alpha\rangle=0$, and hence $s_{\alpha} w \theta=w \theta$, contradicting the fact that $w$ is the shortest element of $W$ that maps $\theta$ to $w \theta$. Thus $w \in{ }^{I} W \cap W^{J}={ }^{I} W^{J}$.

(c) It suffices to show that every relation $\mu<_{\mathrm{B}} \nu$ involving elements $\mu, \nu \in(W \theta)_{I}$ is a transitive consequence of the given relations. For this, let $x, y \in W$ be the shortest elements such that $x \theta=\mu$ and $y \theta=\nu$; we then have $x, y \in W^{J}$ and (by Proposition 1.1) $x<_{\mathrm{B}} y$, so there is a maximal chain $x=x_{0}<_{\mathrm{B}} x_{1}<_{\mathrm{B}} \cdots<_{\mathrm{B}} x_{l}=y$ in $\left(W,<_{\mathrm{B}}\right)$. However, we also have $x, y \in{ }^{I} W$ from (b), and recall that $\left({ }^{I} W,<_{\mathrm{B}}\right)$ and $\left(W^{J},<_{\mathrm{B}}\right)$ are both graded by the length function (cf. Remark $1.2(\mathrm{c})$ ), so we may assume that the maximal chain from $x$ to $y$ is chosen so that each $x_{i}$ is in ${ }^{I} W$. Now since covering relations in $\left(W,<_{\mathrm{B}}\right)$ are generated by reflections, it follows that the image of this chain under the map $w \mapsto w \theta$ is a chain of the desired form, by (a).

REMARK 1.6. (a) The above result shows that $(W \theta)_{I}$ (and hence indirectly, the double quotient ${ }^{I} W^{J}$ ) may be obtained by generating the smallest subset of $V$ that 


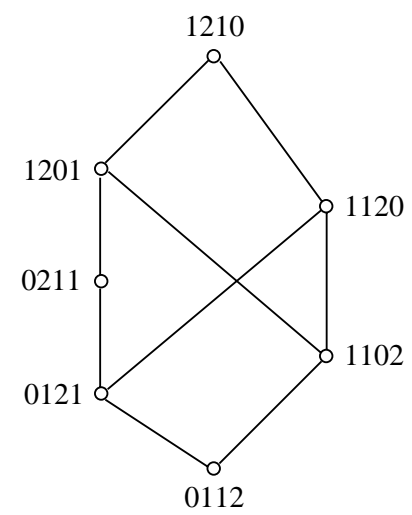

Figure 1: The Bruhat ordering of a double quotient of $S_{4}$.

contains $\theta$ and is closed under the operation of applying a reflection $s_{\beta}(\beta \in \Phi)$ whenever the result stays within the $\Phi_{I}$-dominant chamber. In general, this is vastly more efficient than traversing the full $W$-orbit of $\theta$ and selecting those points that are $\Phi_{I^{-}}$-dominant.

(b) Given $x, y \in{ }^{I} W^{J}$ such that $x<_{\mathrm{B}} y$, there need not exist a maximal chain in $\left(W,<_{\mathrm{B}}\right)$ from $x$ to $y$ such that each intermediate term is also in ${ }^{I} W^{J}$. Otherwise, $\left({ }^{I} W^{J},<_{\mathrm{B}}\right)$ would have to be graded by length, contrary to the example in Figure 1.

EXAmple 1.7. (a) Taking $W$ to be the symmetric group $S_{n}$, acting on $V=\mathbf{R}^{n}$ by permuting coordinates, the Bruhat ordering of an orbit $W \theta$ is generated by transpositions that increase the number of inversions. For a given choice of $I$, the $\Phi_{I}$-dominant part of $W \theta$ consists of those permutations of $\theta$ that have do not have a strict descent at certain fixed positions (depending on $I$ ). For example, if $n=4$ and $\theta=(0,1,1,2)$, there are 7 permutations of $\theta$ that do not have a descent between the first and second positions, and the Bruhat ordering of these 7 points is illustrated in Figure 1.

(b) Another way to index double cosets $W_{I} \backslash W / W_{J}$ in the symmetric group case is to use contingency tables; i.e., nonnegative integer matrices with fixed row and column sums depending on $I$ and $J[\mathbf{D G}]$. Moving to a higher table in the Bruhat ordering corresponds to adding $\left[\begin{array}{rr}-1 & 1 \\ 1 & -1\end{array}\right]$ to some $2 \times 2$ submatrix of a given contingency table.

Returning to the general case, let $\lambda_{I}: W \rightarrow{ }^{I} W$ denote the left sided analogue of the projection $\pi_{I}$; thus, $\lambda_{I}(y x)=x$ for all $y \in W_{I}$ and $x \in{ }^{I} W$. By abuse of notation, we will also use $\lambda_{I}$ to denote a map on $V$ in which $\lambda_{I}(\mu)$ is defined to be the unique member of the $W_{I}$-orbit of $\mu$ that is dominant with respect to $\Phi_{I}$. Proposition $1.5(\mathrm{a})$ shows that these two uses are compatible with evaluation; i.e., $\lambda_{I}(w) \theta=\lambda_{I}(w \theta)$ for all dominant $\theta$ and all $w \in W$.

If we apply left projections to right quotients (a suggestion of Reading), we obtain

Proposition 1.8. Let $I, J \subseteq S$ and assume $\theta \in V$ is dominant with stabilizer $W_{J}$.

(a) We have $\lambda_{I}\left(W^{J}\right)={ }^{I} W^{J}$.

(b) The map $\lambda_{I}:\left(W \theta,<_{\mathrm{B}}\right) \rightarrow\left((W \theta)_{I},<_{\mathrm{B}}\right)$ is order-preserving.

(c) For all $\mu, \nu \in W \theta$, we have $\mu \leqslant_{\mathrm{B}} \nu$ if and only if $\lambda_{\langle s\rangle}(\mu) \leqslant_{\mathrm{B}} \lambda_{\langle s\rangle}(\nu)$ for all $s \in S$. 
Proof. (a) Toward a contradiction, suppose we have $w \in W^{J}$ and $\lambda_{I}(w) \notin W^{J}$. Among all such counterexamples, choose $w$ so as to minimize length. We must have $w \notin{ }^{I} W$; otherwise, $\lambda_{I}(w)=w \in W^{J}$, and $w$ would fail to be a counterexample. Thus $\ell(s w)<\ell(w)$ for some $s \in I$. It follows also that $s w \notin W^{J}$; otherwise, sw would be a shorter counterexample. Hence there is some $t \in J$ such that $\ell(s w t)<\ell(s w)$, and therefore $\ell(w t) \leqslant \ell(s w t)+1=\ell(s w)<\ell(w)$, contradicting the fact that $w \in W^{J}$.

(b) Given $\mu, \nu \in W \theta$, there exist unique $x, y \in W^{J}$ such that $x \theta=\mu$ and $y \theta=\nu$. Since $w \mapsto w^{-1}$ is an automorphism of $\left(W,<_{\mathrm{B}}\right)$ that interchanges $W^{I}$ and ${ }^{I} W$, and right projections are order preserving (Proposition 1.1), it follows that $\lambda_{I}: W \rightarrow{ }^{I} W$ is also order-preserving. Hence,

$$
\mu \leqslant_{\mathrm{B}} \nu \Rightarrow x \leqslant_{\mathrm{B}} y \Rightarrow \lambda_{I}(x) \leqslant_{\mathrm{B}} \lambda_{I}(y) \Rightarrow \lambda_{I}(\mu)=\lambda_{I}(x) \theta \leqslant_{\mathrm{B}} \lambda_{I}(y) \theta=\lambda_{I}(\nu),
$$

the first and third implications being a consequence of Proposition 1.1.

(c) Given $\mu, \nu \in W \theta$ and $x, y \in W^{J}$ as above, (a) implies $\lambda_{\langle s\rangle}(x), \lambda_{\langle s\rangle}(y) \in W^{J}$, so

$$
\lambda_{\langle s\rangle}(\mu) \leqslant_{\mathrm{B}} \lambda_{\langle s\rangle}(\nu) \Rightarrow \lambda_{\langle s\rangle}(x) \leqslant_{\mathrm{B}} \lambda_{\langle s\rangle}(y)
$$

by Proposition 1.1. Again using the fact that $w \mapsto w^{-1}$ is an order automorphism, it follows that there is a left-handed version of Deodhar's criterion (Corollary 1.4). In particular, the above implications for all $s \in S$ combine to imply that $x \leqslant \mathrm{~B} y$, and hence $\mu \leqslant \mathrm{B} \nu$ by evaluation. The converse implication follows from (b).

The above results show that $\left(W^{J},<_{\mathrm{B}}\right)$ is the conjunction of its left projections onto the double quotients $\left({ }^{\langle s\rangle} W^{J},<_{\mathrm{B}}\right)$. More generally, the Bruhat ordering of $W$ or any of its one-sided or double quotients is the conjunction of its projections onto maximal double quotients, and this implies a bound on the order dimension; viz.,

$$
\operatorname{dim}\left({ }^{I} W^{J},<_{\mathrm{B}}\right) \leqslant \sum_{s \in S-I} \sum_{t \in S-J} \operatorname{dim}\left({ }^{\langle s\rangle} W^{\langle t\rangle},<_{\mathrm{B}}\right) .
$$

For example, in the symmetric group case, it is easy to show that the Bruhat ordering of each maximal double quotient is a chain; hence the above bounds immediately yield

$$
\operatorname{dim}\left(S_{n},<_{\mathrm{B}}\right) \leqslant(n-1)^{2} .
$$

Reading has shown that the order dimension of $\left(S_{n},<_{\mathrm{B}}\right)$ is $\left\lfloor n^{2} / 4\right\rfloor$ (see $[\mathbf{R}]$ ).

\section{Tight quotients.}

Having represented the Bruhat orderings of the one-sided and double quotients of $W$ on the $W$-orbits of various (dominant) points $\theta$ in a real vector space $V$, it is natural to investigate the extent to which these representations may be used provide a coordinate embedding of the corresponding posets. 
Recall that if $\mu<_{\mathrm{B}} \nu$ is a covering relation in $\left(W \theta,<_{\mathrm{B}}\right)$, then $\mu-\nu$ is a positive multiple of a positive root, and thus in the (simplicial) cone $\mathbf{R}^{+} \Phi^{+}$generated by the positive roots. We define the standard (or root) ordering of $V$ to be the partial order

$$
\mu \succcurlyeq \nu \quad \text { if } \mu-\nu \in \mathbf{R}^{+} \Phi^{+} \text {. }
$$

In these terms, the Bruhat order is consistent with the dual of the standard order; i.e.,

$$
\mu \leqslant \mathrm{B} \nu \Rightarrow \mu \succcurlyeq \nu
$$

If this is an order embedding (i.e., $\mu \leqslant_{\mathrm{B}} \nu \Leftrightarrow \mu \succcurlyeq \nu$ for all $\mu, \nu \in W \theta$ ), then we say that the Bruhat ordering of $W \theta$ is tight, or simply that $\left(W \theta,<_{\mathrm{B}}\right)$ is tight. More generally, any subposet of $\left(W \theta,<_{\mathrm{B}}\right)$ with this property is also said to be tight. In particular, the orbit representation of the Bruhat ordering of some double quotient, say $\left((W \theta)_{I},<_{\mathrm{B}}\right)$, is tight if $\mu \leqslant_{\mathrm{B}} \nu \Leftrightarrow \mu \succcurlyeq \nu$ for all $\mu, \nu \in(W \theta)_{I}$.

For example, consider the double quotient of $S_{4}$ discussed in Example 1.7(a) and illustrated in Figure 1. The pair $\mu=(0,2,1,1)$ and $\nu=(1,1,2,0)$ are incomparable in the Bruhat order, and yet $\mu-\nu=(-1,1,-1,1)$ is the sum of two simple roots, so we have $\mu \succ \nu$. This representation of the double quotient is therefore not tight.

REMARK 2.1. (a) The simple roots generate the cone $\mathbf{R}^{+} \Phi^{+}$, so if $X \subseteq W \theta$ is tight, then the coordinates of $\theta-\mu$ with respect to the simple roots, as $\mu$ varies over $X$, provide a coordinate embedding of the Bruhat ordering of $X$. In particular, the order dimension of any tight subposet of the Bruhat order is at most $|S|$.

(b) If we renormalize the root system $\Phi$, independently replacing each $W$-orbit of roots by some positive scalar multiple, then the dominant chamber, the set of $W$ orbits in $V$, the cone spanned by the positive roots, and the standard ordering are all unchanged. Thus, tightness does not depend on how the root system is normalized.

Example 2.2. It is implicit in the work of Proctor (see Proposition 4.1 of $[\mathbf{P 2}]$ ) that if $W$ is finite and $\theta$ is minuscule (i.e., $\left\langle\theta, \beta^{\vee}\right\rangle \in\{0, \pm 1\}$ for all roots $\beta$ ), then the Bruhat ordering of $W \theta$ is tight. Indeed, if $\theta$ is minuscule, then the same is true for every point in the $W$-orbit of $\theta$, and thus every reflection acts on $W \theta$ by adding (or subtracting) a root. It follows that if $\mu \succ \nu$ in $W \theta$, then $\mu-\nu=\sum c_{i} \alpha_{i}$ for certain positive integers $c_{i}$ and simple roots $\alpha_{i}$. Furthermore, given that $W$ is finite, it is necessarily the case that $\langle$,$\rangle is positive definite on \operatorname{Span} \Phi$, and hence $\left\langle\mu-\nu, \alpha_{i}^{\vee}\right\rangle>0$ for some $i$, whence $\left\langle\mu, \alpha_{i}^{\vee}\right\rangle=1$ or $\left\langle\nu, \alpha_{i}^{\vee}\right\rangle=-1$. Thus we obtain $\mu<_{\mathrm{B}} \mu-\alpha_{i} \succcurlyeq \nu$ or $\mu \succcurlyeq \nu+\alpha_{i}<_{\mathrm{B}} \nu$ and it follows by induction with respect to $\sum c_{i}$ that $\mu<_{\mathrm{B}} \nu$.

THEOREM 2.3. The Bruhat ordering of $W \theta$ is tight if and only if for all $s \in S$, the Bruhat ordering of the double quotient $(W \theta)_{\langle s\rangle}$ is a chain.

Our proof will rely on the following pair of lemmas. The first of these may be wellknown, but we have not seen it in the literature.

Lemma 2.4. If $\Phi$ is infinite and irreducible, then for every $\mu \in$ Span $\Phi$, there exist roots $\gamma$ such that $\gamma \succ \mu$. 
Proof. Consider the collection of subsets $J \subseteq S$ such that the claimed property is true for all $\mu \in \operatorname{Span} \Phi_{J}$. There must be nonempty sets $J$ with this property; otherwise, the coordinates of the positive roots would be bounded, and hence $\Phi$ would have an accumulation point. In turn, this would force the set of reflections in $W$ to have an accumulation point in the usual topology of $G L(V)$, contradicting the fact that Coxeter groups are discrete (e.g., see Section 6.2 of $[\mathbf{H}]$ ).

Now suppose that $J$ is maximal with respect to the above property. Given that $\Phi$ is irreducible (and assuming $J \neq S$ ), there must exist simple reflections $s_{\alpha} \in J$ and $s_{\beta} \notin J$ such that the corresponding nodes of the Coxeter diagram are adjacent; i.e., $\langle\alpha, \beta\rangle<0$. However since $J$ is maximal, there must be some $\mu \in \operatorname{Span} \Phi_{J}$ and a scalar $c>0$ such that there is no root $\gamma \succ \mu+c \beta$. On the other hand, we have $s_{\alpha} \in J$, so we can find roots $\gamma \succ \mu+t \alpha$ for all $t>0$. The coefficient of $\beta$ in all such roots must necessarily be $\leqslant c$, and since $\left\langle\alpha^{\prime}, \beta\right\rangle \leqslant 0$ for all simple roots $\alpha^{\prime} \neq \beta$, it follows that $\left\langle\gamma, \beta^{\vee}\right\rangle \leqslant 2 c+t\left\langle\alpha, \beta^{\vee}\right\rangle$. By choosing $t$ sufficiently large, we force $\left\langle\gamma, \beta^{\vee}\right\rangle<-c$, whence

$$
s_{\beta}(\gamma) \succ \gamma+c \beta \succ \mu+c \beta
$$

contradicting the choice of $\mu$ and $c$.

Lemma 2.5. Assume $W$ is irreducible. If $\mu, \nu \in V$ are dominant and $\mu-\nu \in \operatorname{Span} \Phi$, then there exist points $\mu^{\prime}$ in the $W$-orbit of $\mu$ such that $\nu \succcurlyeq \mu^{\prime}$, except possibly if $W$ is infinite and acts trivially on $\mu$.

Proof. If $\Phi$ (and hence $W$ ) is finite, then there is an anti-dominant member of the $W$-orbit of $\mu$, say $\mu^{\prime}$. If $\nu \succcurlyeq \mu^{\prime}$ failed, then there would be a nonempty set of simple roots $\alpha$ with negative coefficients in $\nu-\mu^{\prime}$. Choosing $\beta$ to be a (necessarily positive) root that is dominant relative to the corresponding (finite) parabolic root subsystem $\Phi_{J}$, we would therefore have $\langle\alpha, \beta\rangle \leqslant 0$ for simple roots $\alpha$ not in $\Phi_{J}$ (since $\left\langle\alpha, \alpha^{\prime}\right\rangle \leqslant 0$ for distinct simple roots) and $\langle\alpha, \beta\rangle \geqslant 0$ for simple roots $\alpha$ in $\Phi_{J}$, with at least one strict inequality among the latter cases. It follows that $\left\langle\nu-\mu^{\prime}, \beta\right\rangle<0$, so either $\langle\nu, \beta\rangle<0$ or $\left\langle\mu^{\prime}, \beta\right\rangle>0$, contradicting the fact that $\nu$ is dominant and $\mu^{\prime}$ is anti-dominant.

If $\Phi$ is infinite, then by Lemma 2.4 there is a positive $\operatorname{root} \beta$ such that $\nu \succcurlyeq \mu-\beta$. Also, given that $W$ does not act trivially on $\mu$, there is a (simple) root $\alpha$ such that $\langle\mu, \alpha\rangle>0$. Replacing $\beta$ with a higher root if necessary, we may therefore assume that $\left\langle\mu, \beta^{\vee}\right\rangle \geqslant 1$, and hence $\nu \succcurlyeq \mu-\beta \succcurlyeq s_{\beta}(\mu)$.

We remark that the above lemma fails without the exception for trivial actions. For example, if the bilinear form $\langle$,$\rangle is degenerate on the span of \Phi$, then there exist nonzero $W$-fixed points $\delta \in \operatorname{Span} \Phi$. It cannot be the case that both $\delta \succcurlyeq 0$ and $0 \succcurlyeq \delta$, so taking either $\mu=\delta, \nu=0$ or $\mu=0, \nu=\delta$ would produce a counterexample.

Proof of Theorem 2.3. $(\Rightarrow)$ Suppose $\mu, \nu \in(W \theta)_{\langle s\rangle}$ are incomparable in the Bruhat order. Interchanging $\mu$ and $\nu$ if necessary, we may assume that the coefficient of $\alpha$ in $\nu-\mu$ is nonnegative, where $\alpha$ denotes the simple root corresponding to $s$. We claim that there is an element $\mu^{\prime}$ in the $W_{\langle s\rangle}$-orbit of $\mu$ such that $\nu \succcurlyeq \mu^{\prime}$. If this failed, then by Lemma 2.5 , there would have to be an infinite irreducible component $W_{J}$ of $W_{\langle s\rangle}$ 
that acts trivially on $\mu$, and a simple root $\alpha^{\prime} \in \Phi_{J}$ such that the coefficient of $\alpha^{\prime}$ in $\nu-\mu$ is negative. Note that $W_{J \cup\{s\}}$ must also be irreducible; otherwise, $W_{J}$ would be an irreducible component of $W$ and the coefficient of $\alpha^{\prime}$ would be constant on $W \theta$.

Now since $\mu$ is dominant with respect to $\Phi_{\langle s\rangle}$ but not $\Phi$-dominant (otherwise $\mu=\theta$ and we contradict the fact that $\mu$ and $\nu$ are incomparable), it must be the case that $\langle\mu, \alpha\rangle<0$. We also have $\langle\mu, \beta\rangle=0$ for $\beta \in \Phi_{J}$, so $\langle\mu, \beta\rangle<0$ all positive roots $\beta$ in $\Phi_{J \cup\{s\}}-\Phi_{J}$. However, there are infinitely many such roots (Lemma 2.4), contradicting the fact that the number of these roots is bounded by the rank of $\mu$ (see Remark 1.2(c)).

Given that $\left(W \theta,<_{\mathrm{B}}\right)$ is tight, we may conclude that $\nu \leqslant_{\mathrm{B}} \mu^{\prime}$, and hence $\nu \leqslant_{\mathrm{B}} \mu$, since the left projection map $\lambda_{\langle s\rangle}$ preserves the Bruhat order (Proposition 1.8(b)). However, this contradicts the hypothesis that $\mu$ and $\nu$ are incomparable.

$(\Leftarrow)$ Given $\mu, \nu \in W \theta$ such that $\mu \succcurlyeq \nu$, the left projections $\lambda_{\langle s\rangle}(\mu)$ and $\lambda_{\langle s\rangle}(\nu)$ must be Bruhat-related for all $s \in S$, since each of $\left((W \theta)_{\langle s\rangle},<_{\mathrm{B}}\right)$ is a chain. We cannot have $\lambda_{\langle s\rangle}(\nu)<_{\mathrm{B}} \lambda_{\langle s\rangle}(\mu)$; otherwise, since $\lambda_{\langle s\rangle}(\mu)$ is the Bruhat-minimum of its $W_{\langle s\rangle^{-}}$ orbit, the first step in any Bruhat-decreasing chain from $\lambda_{\langle s\rangle}(\mu)$ to $\lambda_{\langle s\rangle}(\nu)$ would be a reflection not in $W_{\langle s\rangle}$, and thus the simple root $\alpha$ corresponding to $s$ would appear in $\lambda_{\langle s\rangle}(\mu)-\lambda_{\langle s\rangle}(\nu)$ with a coefficient $<0$. However, the coefficient of $\alpha$ is constant on $W_{\langle s\rangle}$-orbits, so this contradicts the fact that $\mu \succcurlyeq \nu$. Thus we have $\lambda_{\langle s\rangle}(\mu) \leqslant_{\mathrm{B}} \lambda_{\langle s\rangle}(\nu)$ for all $s \in S$, and hence $\mu \leqslant_{\mathrm{B}} \nu$ by Deodhar's criterion (Proposition 1.8(c)).

REMARK 2.6. (a) Theorem 2.3 shows that the tightness of a one-sided quotient is a purely order-theoretic property, and thus depends only on the generating set $J$ for the stabilizer of $\theta$. In other words, we may define $\left(W^{J},<_{\mathrm{B}}\right)$ to be tight if and only if $\left(W \theta,<_{\mathrm{B}}\right)$ is tight for some (equivalently, every) dominant $\theta \in V$ with stabilizer $W_{J}$.

(b) On the other hand, the tightness of the Bruhat ordering of an orbit representation of a double quotient is not purely a property of the underlying poset. For example, suppose $W=E_{8}$ and that $s, t \in S$ are the simple reflections such that $W_{\langle s\rangle} \cong D_{7}$ and $W_{\langle t\rangle} \cong A_{7}$. If $\theta_{s}$ and $\theta_{t}$ are any dominant points whose stabilizers are $W_{\langle s\rangle}$ and $W_{\langle t\rangle}$, then it happens that $\left(W \theta_{s}\right)_{\langle t\rangle}$ is tight and $\left(W \theta_{t}\right)_{\langle s\rangle}$ is not, even though the Bruhat orderings of $\left(W \theta_{s}\right)_{\langle t\rangle}$ and $\left(W \theta_{t}\right)_{\langle s\rangle}$ are necessarily isomorphic.

QUESTION 2.7. Is the infinite dihedral group the only (irreducible) infinite Coxeter group $W$ with a nontrivial quotient $\left(W^{J},<_{\mathrm{B}}\right)$ that is tight?

Theorem 4.9 below answers this question affirmatively for the affine Weyl groups.

\section{Tight quotients of finite Coxeter groups.}

In this section, we assume that $W$ (and hence $\Phi$ ) is finite. One knows in this case that $\langle$,$\rangle is necessarily positive definite on the span of \Phi$, and there is no harm in assuming that it is positive definite on all of $V$, whether or not $\Phi$ spans $V$.

It will be convenient to explicitly name the simple reflections $s_{1}, \ldots, s_{n}$ and the corresponding simple roots $\alpha_{1}, \ldots, \alpha_{n}$. We will also modify the notation of Section 1 slightly by setting $\langle i\rangle:=S-\left\{s_{i}\right\}$.

We let $\omega_{1}, \ldots, \omega_{n}$ denote the fundamental weights; i.e., the basis of Span $\Phi$ dual to the simple co-roots. Thus $\left\langle\omega_{i}, \alpha_{j}^{\vee}\right\rangle=\delta_{i j}$ for all $i$ and $j, \omega_{i}$ is dominant, and the $W$-stabilizer of $\omega_{i}$ is the parabolic subgroup $W_{\langle i\rangle}$. 


\section{A. Orbit stratification and the double weight arrangement.}

A useful necessary condition for $\left(W \theta,<_{\mathrm{B}}\right)$ to be tight involves the stratification of sub-orbits generated by parabolic subgroups. More specifically, the parabolic subgroup $W_{\langle i\rangle}$ fixes $\omega_{i}$, and hence the functional $\mu \mapsto\left\langle\mu, \omega_{i}\right\rangle$ (i.e., the coefficient of $\alpha_{i}^{\vee}$ in $\mu$ ) is constant on $W_{\langle i\rangle}$-orbits. If this functional separates the $W_{\langle i\rangle}$-orbits in $W \theta$ (i.e., $\mu, \nu \in W \theta$ are in the same $W_{\langle i\rangle}$-orbit if and only if the coefficients of $\alpha_{i}^{\vee}$ in $\mu$ and $\nu$ are the same), then we say that the $W_{\langle i\rangle}$-orbits in $W \theta$ are stratified.

Proposition 3.1. If $\left(W \theta,<_{\mathrm{B}}\right)$ is tight, then the $W_{\langle i\rangle}$-orbits in $W \theta$ are stratified.

Proof. Suppose we have $\mu, \nu \in W \theta$ such that the coefficient of $\alpha_{i}^{\vee}$ in $\mu-\nu$ is zero. Without loss of generality, we may assume that $\mu$ and $\nu$ are dominant relative to $\Phi_{\langle i\rangle}$; i.e., $\mu, \nu \in(W \theta)_{\langle i\rangle}$. Given that $\left(W \theta,<_{\mathrm{B}}\right)$ is tight, we know that the Bruhat ordering of $(W \theta)_{\langle i\rangle}$ must be a chain (Theorem 2.3), and hence $\mu$ and $\nu$ must be related; say $\mu \leqslant_{\mathrm{B}} \nu$. However, each Bruhat-increasing application of a reflection subtracts a positive multiple of a positive root, and $\mu-\nu \in \operatorname{Span} \Phi_{\langle i\rangle}$, so this is possible only if every chain from $\mu$ to $\nu$ is generated by reflections corresponding to roots in $\Phi_{\langle i\rangle}$. In turn, this forces $\mu$ and $\nu$ into the same $W_{\langle i\rangle}$-orbit, and hence $\mu=\nu$.

REMARK 3.2. (a) It is not hard to see that the above property cannot characterize tightness. For example, the $W_{\langle i\rangle}$-orbits in a sufficiently generic $W$-orbit are always stratified, but according to the classification below, the Bruhat ordering of a generic $W$-orbit is tight only if $W$ is of rank $\leqslant 2$. On the other hand, it turns out that a slightly stronger form of orbit stratification does characterize tightness - see Corollary 3.10 below.

(b) Since $W_{\langle i\rangle}$-orbits in $W \theta$ are in bijection with double cosets (Proposition 1.5(b)), it follows that stratification of $W_{\langle i\rangle}$-orbits may be tested by verifying that the number of distinct values for $\left\langle\mu, \omega_{i}\right\rangle$, as $\mu$ varies over $W \theta$, equals the number of double cosets $W_{\langle i\rangle} \backslash W / W_{J}$, where $W_{J}$ denotes the stabilizer of $\theta$. In turn, one may count these double cosets by computing the character inner product for the permutation actions of $W$ on the orbits of $\omega_{i}$ and $\theta$. In practice, it is usually easier to explicitly generate $(W \theta)_{\langle i\rangle}$ by the algorithm suggested in Remark 1.6(a), and test stratification directly.

Once the tight quotients $\left(W^{\langle j\rangle},<_{\mathrm{B}}\right)$ have been classified, we claim that the tightness of $\left(W^{J},<_{\mathrm{B}}\right)$ may be reduced to a geometric question relating the $J$-th face of the dominant chamber to the faces ${ }^{2}$ of the hyperplane arrangement $\mathcal{H}=\mathcal{H}(\Phi)$ whose normal vectors are the nonzero vectors of the form

$$
x \omega_{i}-y \omega_{i} \quad(x, y \in W, 1 \leqslant i \leqslant n) .
$$

We refer to this as the double weight arrangement associated to $\Phi$.

Lemma 3.3. The Bruhat ordering of $W^{J}$ is tight if and only if

(i) $\left(W \omega_{j},<_{\mathrm{B}}\right)$ is tight for all $s_{j} \in S-J$, and

(ii) there is a face of $\mathcal{H}$ that contains every dominant $\theta \in V$ with stabilizer $W_{J}$.

\footnotetext{
${ }^{2}$ By a face of the hyperplane arrangement $\left\{H_{1}, \ldots, H_{l}\right\}$, we mean a non-empty intersection of the form $H_{1}\left(\varepsilon_{1}\right) \cap \cdots \cap H_{l}\left(\varepsilon_{l}\right)$, where $\varepsilon_{i} \in\{-, 0,+\}, H(0)=H$, and $H(+)$ and $H(-)$ denote the two half spaces in the complement of $H$.
} 
Proof. Given that $\left(W^{J},<_{\mathrm{B}}\right)$ is tight, it follows via Theorem 2.3 that $\left({ }^{\langle i\rangle} W^{J},<_{\mathrm{B}}\right)$ is a chain, say $w_{1}<_{\mathrm{B}} w_{2}<_{\mathrm{B}} \cdots<_{\mathrm{B}} w_{m}$. Hence $w_{1} \theta \succ w_{2} \theta \succ \cdots \succ w_{m} \theta$ for all dominant $\theta$ with stabilizer $W_{J}$, and therefore

$$
\left\langle w_{1} \theta, \omega_{i}\right\rangle>\left\langle w_{2} \theta, \omega_{i}\right\rangle>\cdots>\left\langle w_{m} \theta, \omega_{i}\right\rangle .
$$

We must have strict inequality here, or else the $W_{\langle i\rangle}$-orbits in $W \theta$ would fail to stratify, contradicting Proposition 3.1. Now since the value of $\left\langle w \theta, \omega_{i}\right\rangle=\left\langle\theta, w^{-1} \omega_{i}\right\rangle$ is unchanged if we replace $w$ with any member of the double coset $W_{\langle i\rangle} w W_{J}$, it follows that if $x^{-1}$ and $y^{-1}$ are in the same double cosets as $w_{k}$ and $w_{l}$ (respectively), then

$$
\left\langle\theta, x \omega_{i}\right\rangle \geqslant\left\langle\theta, y \omega_{i}\right\rangle \Leftrightarrow k \leqslant l,
$$

and equality occurs if and only if $k=l$. Thus $\theta$ is confined to a single face of $\mathcal{H}$.

Also, for every $s_{j} \in S-J$, we have $W^{\langle j\rangle} \subseteq W^{J}$, so $\left({ }^{\langle i\rangle} W^{\langle j\rangle},<_{\mathrm{B}}\right)$ is a subposet of $\left({ }^{\langle i\rangle} W^{J},<_{\mathrm{B}}\right)$; i.e., a chain. Hence $\left(W \omega_{j},<_{\mathrm{B}}\right)$ is tight, by Theorem 2.3 .

Conversely, given that (i) and (ii) hold, choose any dominant $\theta \in V$ with stabilizer $W_{J}$ and suppose there exist $\mu, \nu \in W \theta$ with $\mu \succcurlyeq \nu$ but $\mu \Varangle_{\mathrm{B}} \nu$. We have $\mu=x \theta$ and $\nu=y \theta$ for some (unique) $x, y \in W^{J}$, hence $x \mathbb{B}_{\mathrm{B}} y$ (Proposition 1.1(b)), and thus $x \omega_{j} \mathbb{B}_{\mathrm{B}} y \omega_{j}$ for some $j$ such that $s_{j} \in S-J$ (Corollary 1.4). Given the hypothesis that $\left(W \omega_{j},<_{\mathrm{B}}\right)$ is tight, it follows that $x \omega_{j} \nsucc y \omega_{j}$, and hence $\left\langle x \omega_{j}-y \omega_{j}, \omega_{i}\right\rangle<0$ for some $i$.

Now let $\theta(t):=t \theta+(1-t) \omega_{j}(0 \leqslant t \leqslant 1)$ parameterize the line segment from $\omega_{j}$ to $\theta$. It is easy to see that $\theta(t)$ is dominant and has stabilizer $W_{J}$ for $t>0$. However,

$$
\left\langle\theta(t), x^{-1} \omega_{i}-y^{-1} \omega_{i}\right\rangle=\left\langle x \theta(t)-y \theta(t), \omega_{i}\right\rangle
$$

is a linear function of $t$ that is $<0$ for $t=0$ and $\geqslant 0$ for $t=1$ (since $\mu \succcurlyeq \nu$ ), so there must be some $t>0$ such that $\theta(t)$ lies on the negative side of the hyperplane orthogonal to $x^{-1} \omega_{i}-y^{-1} \omega_{i}$ and $\theta$ does not, contradicting (ii).

Since Coxeter diagrams are acyclic, the following result shows (via Lemma 3.3) that if $\left(W^{J},<_{\mathrm{B}}\right)$ is tight and $W$ is irreducible, then $W^{J}$ has co-rank at most 2 (i.e., $\left|J^{c}\right| \leqslant 2$ ).

LEMma 3.4. If $W$ is irreducible and there is a face of $\mathcal{H}$ that contains all dominant $\theta$ with stabilizer $W_{J}$, then every pair $s_{i}, s_{j} \in S-J$ is adjacent in the Coxeter diagram, and if the edge between $i$ and $j$ is simple (i.e., $\left(s_{i} s_{j}\right)^{3}=1$ ), then $W$ is of type $A$.

Proof. If $W$ is not of type $A$ and not a dihedral group $I_{2}(m)$ with $m$ odd, then every orbit of roots is generated by a fundamental weight or a scalar multiple thereof (depending on the normalization). For the crystallographic cases, this is equivalent to the fact that in the extended Dynkin diagram, the "extra" node is adjacent to only one node except in type $A$. For the non-crystallographic groups $I_{2}(m), H_{3}$, and $H_{4}$, this is an easy calculation.

Now suppose that there exist (positive) roots $\beta_{i}$ and $\beta_{j}$ in the same $W$-orbit such that $\left\langle\omega_{i}, \beta_{i}\right\rangle>0,\left\langle\omega_{j}, \beta_{j}\right\rangle>0$, and $\left\langle\omega_{i}, \beta_{j}\right\rangle=\left\langle\omega_{j}, \beta_{i}\right\rangle=0$. If so, then for all sufficiently small dominant $\theta$ with stabilizer $W_{J}$, the quantity

$$
\left\langle\theta+t \omega_{i}+(1-t) \omega_{j}, \beta_{i}-\beta_{j}\right\rangle
$$


is negative for small $t>0$ and positive for large $t<1$, and thus the set of dominant vectors with stabilizer $W_{J}$ is not confined to one side of the hyperplane orthogonal to $\beta_{i}-\beta_{j}$. However the preceding discussion shows that if $W$ is of rank $>2$ and not of type $A$, then $\beta_{i}-\beta_{j}$ is one of the normal vectors for the arrangement $\mathcal{H}$, a contradiction.

We may take $\beta_{i}=\alpha_{i}$ and $\beta_{j}=\alpha_{j}$ provided that $\alpha_{i}$ and $\alpha_{j}$ are in the same $W$-orbit; i.e., nodes $i$ and $j$ must be connected by a path in the Coxeter graph that avoids edges of even weight. This eliminates all cases except those of type $A$ and those in which the unique path from $i$ to $j$ passes through a (necessarily unique) edge of weight $>3$.

In the latter case, it suffices to show that $i$ and $j$ must be adjacent. If this fails, then either $i$ or $j$ must fail to be an endpoint of the edge of weight $>3$ (or both). Interchanging $i$ and $j$ if necessary, we assume that $i$ has this property, and that $k$ is the first node on the unique path from $i$ to $j$. In that case, $\alpha_{i}$ and $\alpha_{k}$ are in the same $W$-orbit, and $\alpha_{k}$ and $\alpha_{j}$ are in the same irreducible component of $\Phi_{\langle i\rangle}$, so we may take $\beta_{i}=\alpha_{i}$ and $\beta_{j}$ to be the root in the $W_{\langle i\rangle}$-orbit of $\alpha_{k}$ that is dominant relative to $\Phi_{\langle i\rangle}$.

The remaining possibility is that $W$ is of type $A$. Although not all root differences $\beta_{i}-\beta_{j}$ are normal vectors for the double weight arrangement in type $A$, it happens that the normal vectors do include the differences of orthogonal roots. Indeed, one may check that all such differences form a single $W$-orbit in $\left\{x \omega_{2}-y \omega_{2}: x, y \in W\right\}$. Thus if $i$ and $j$ are not adjacent, the hyperplane orthogonal to $\alpha_{i}-\alpha_{j}$ is in $\mathcal{H}$, so we may again reach a contradiction by choosing $\beta_{i}=\alpha_{i}$ and $\beta_{j}=\alpha_{j}$.

\section{B. The classification.}

In order to identify the tight quotients of finite Coxeter groups, we first collect some additional necessary conditions related to orbit stratification.

LEMMA 3.5. If $\Psi \subseteq \Phi$ is an orbit of roots that includes two orthogonal simple roots, then for some $k$, the $W_{\langle k\rangle}$-orbits in $\Psi$ are not stratified (and thus $\left(\Psi,<_{\mathrm{B}}\right)$ is not tight).

Proof. If $\alpha_{i}$ and $\alpha_{j}$ are in the same $W$-orbit, then nodes $i$ and $j$ in the Coxeter diagram are connected by a path. Given that $\alpha_{i}$ and $\alpha_{j}$ are orthogonal, nodes $i$ and $j$ must also be non-adjacent. Now since the diagrams of finite Coxeter groups are acyclic, there must be an intermediate node $k$ along a path connecting $i$ and $j$ whose removal leaves $i$ and $j$ in separate components. Thus $\alpha_{i}$ and $\alpha_{j}$ belong to distinct irreducible components of $\Phi_{\langle k\rangle}$, and hence in distinct $W_{\langle k\rangle}$-orbits. On the other hand, the coefficients of $\alpha_{k}$ in $\alpha_{i}$ and $\alpha_{j}$ are both zero, so the $W_{\langle k\rangle}$-orbits in $\Psi$ cannot be stratified.

Once a particular $W$-orbit is known to violate stratification, the following result allows us to extend the violation to orbits of larger Coxeter groups.

LEMMA 3.6. If $\theta$ is dominant and the $W_{\langle j\rangle}$-orbits in $W \theta$ are stratified, then the $W_{J \cap\langle j\rangle}$-orbits in $W_{J} \theta$ are stratified for all $J$ such that $\left\{s_{j}\right\} \subseteq J \subseteq S$.

Proof. Suppose $\mu, \nu \in W_{J} \theta$ and that the coefficient of $\alpha_{j}$ in $\mu-\nu$ is zero. Without loss of generality, we may assume that $\mu$ and $\nu$ are both dominant relative to $\Phi_{J \cap\langle j\rangle}$. Since $\theta$ is the maximum element of the standard ordering of $W_{J} \theta$, we have $\mu=\theta-\gamma$ for some $\gamma$ in the nonnegative span of $\Phi_{J}^{+}$. Moreover, since $\left\langle\alpha_{i}, \alpha_{k}\right\rangle \leqslant 0$ for $i \neq k$, it follows that $\gamma$ is anti-dominant relative to $\Phi_{J^{c}}$, and thus $\mu$ and (similarly) $\nu$ are dominant relative 

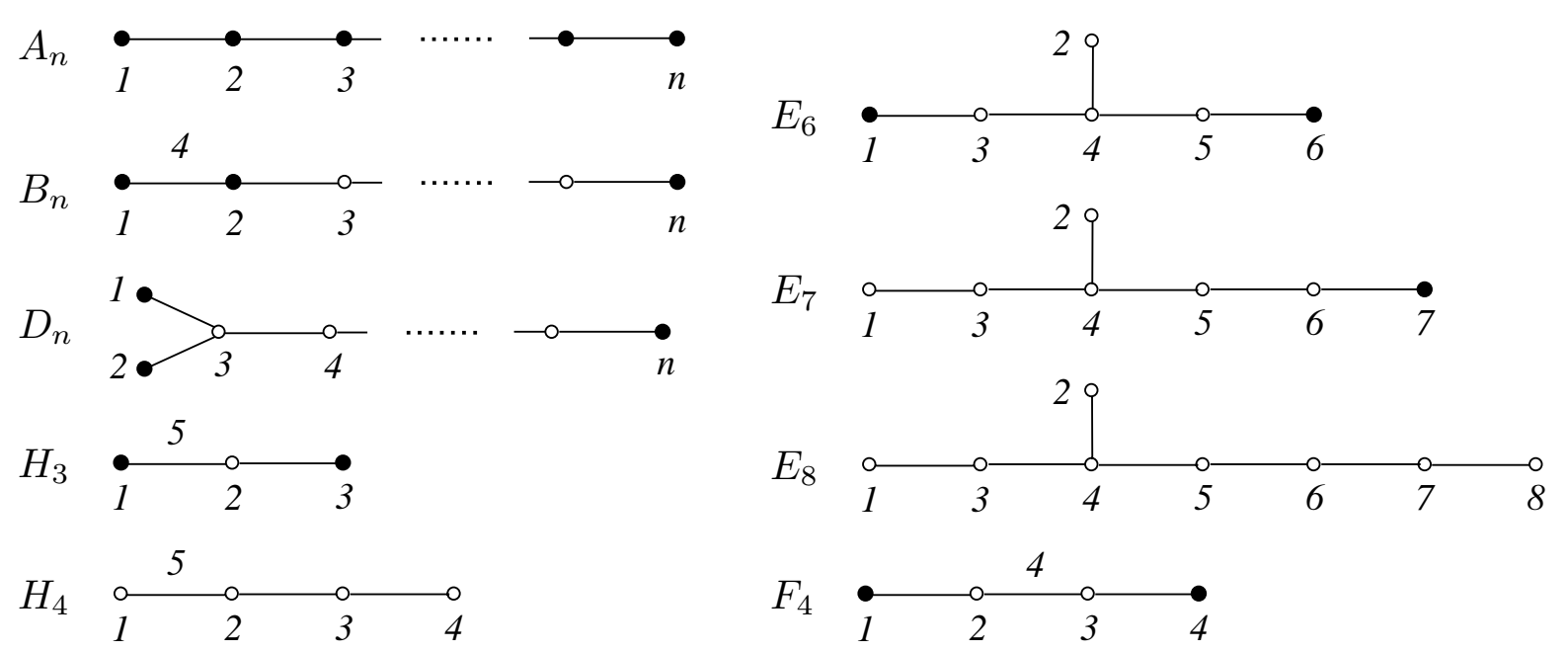

FIGURE 2: The maximal tight quotients ( $\operatorname{rank} W \geqslant 3 ; \bullet=$ tight).

to $\Phi_{\langle j\rangle}$. Given that the $W_{\langle j\rangle}$-orbits in $W \theta$ are stratified, this is possible only if $\mu$ and $\nu$ are in the same $W_{\langle j\rangle}$-orbit; since both are $\Phi_{\langle j\rangle}$-dominant, this forces $\mu=\nu$.

LEMma 3.7. If the $W_{\langle i\rangle}$-orbits in $W \omega_{j}$ are stratified, then the $W_{\langle j\rangle}$-orbits in $W \omega_{i}$ are also stratified.

Proof. Suppose $\mu, \nu \in W \omega_{i}$ satisfy $\left\langle\mu, \omega_{j}\right\rangle=\left\langle\nu, \omega_{j}\right\rangle$. We may choose $x, y \in W$ so that $\mu=x \omega_{i}$ and $\nu=y \omega_{i}$, whence

$$
\left\langle\omega_{i}, x^{-1} \omega_{j}\right\rangle=\left\langle\mu, \omega_{j}\right\rangle=\left\langle\nu, \omega_{j}\right\rangle=\left\langle\omega_{i}, y^{-1} \omega_{j}\right\rangle
$$

Given that the $W_{\langle i\rangle}$-orbits in $W \omega_{j}$ are stratified, this forces $x^{-1} \omega_{j}$ and $y^{-1} \omega_{j}$ to be in the same $W_{\langle i\rangle}$-orbit, and thus $w x^{-1} \omega_{j}=y^{-1} \omega_{j}$ for some $w \in W_{\langle i\rangle}$. However, $W_{\langle j\rangle}$ is the stabilizer of $\omega_{j}$, so we have $y w x^{-1} \in W_{\langle j\rangle}$, hence $y w \in W_{\langle j\rangle} x$, and therefore $\nu=y w \omega_{i}$ is in the same $W_{\langle j\rangle}$-orbit as $\mu=x \omega_{i}$.

We are now ready for the classification of tight one-sided quotients.

THEOREM 3.8. If $W$ is finite and irreducible, then $\left(W^{J},<_{\mathrm{B}}\right)$ is tight if and only if $W$ is of rank at most 2 , or $J=S$, or one of the following holds:

- $W \cong A_{n}$ and $J^{c}=\left\{s_{j}\right\}(1 \leqslant j \leqslant n)$ or $J^{c}=\left\{s_{j}, s_{j+1}\right\}(1 \leqslant j<n)$,

- $W \cong B_{n}$ and $J^{c}=\left\{s_{1}\right\},\left\{s_{2}\right\},\left\{s_{n}\right\}$, or $\left\{s_{1}, s_{2}\right\}$,

- $W \cong D_{n}$ and $J^{c}=\left\{s_{1}\right\},\left\{s_{2}\right\}$, or $\left\{s_{n}\right\}$,

- $W \cong E_{6}$ and $J^{c}=\left\{s_{1}\right\}$ or $\left\{s_{6}\right\}$,

- $W \cong E_{7}$ and $J^{c}=\left\{s_{7}\right\}$,

- $W \cong F_{4}$ and $J^{c}=\left\{s_{1}\right\}$ or $\left\{s_{4}\right\}$, or

- $W \cong H_{3}$ and $J^{c}=\left\{s_{1}\right\}$ or $\left\{s_{3}\right\}$.

The numbering of the simple reflections in each case is indicated in Figure 2. 
Proof. The Bruhat ordering of every nontrivial quotient of a dihedral group is a chain, so all such Coxeter groups and their quotients have tight Bruhat orders by Theorem 2.3. Also, we have previously noted (see Example 2.2) that if $\theta$ is minuscule with respect to $\Phi$ or one of its renormalizations, then $\left(W \theta,<_{\mathrm{B}}\right)$ is tight. Bearing in mind the classification of minuscule weights (e.g., see Exercise VI.4.15 of $[\mathbf{B}]$ ), this accounts for all of the cases listed above with $\left|J^{c}\right|=1$ except for those involving $F_{4}, H_{3}$, and the $B_{n}$-orbit of $\omega_{2}$.

The case $W=F_{4}$. Let $\Psi$ denote the orbit of short roots in a finite crystallographic root system. It is well-known and easy to show that if $\alpha$ covers $\beta$ in the standard ordering of $\Psi$, and $\beta$ is positive, then $\alpha-\beta$ must be a simple root (e.g., Proposition 3.2 of [S3]), and hence $\alpha<_{\mathrm{B}} \beta$ (cf. Remark 1.2(b)). Dual reasoning shows that the same must be true if $\alpha$ is negative. The remaining possibility for a covering relation in the standard order is that $\alpha$ is positive and $\beta$ is negative, in which case $\alpha=\alpha_{i}$ and $\beta=-\alpha_{j}$ for some $i$ and $j$. If the corresponding nodes are adjacent in the Coxeter diagram, then $\alpha-\beta=\alpha_{i}+\alpha_{j}$ is a (positive) root, and hence $\alpha_{i}<_{\mathrm{B}}-\alpha_{j}$. Thus if all pairs of nodes corresponding to short simple roots are adjacent (in particular, this means that there can be at most two short simple roots), then $\left(\Psi,<_{\mathrm{B}}\right)$ is tight. For the two cases in question, the $F_{4}$-orbits of $\omega_{1}$ and $\omega_{4}$ are both root orbits; each has exactly two simple roots, the corresponding nodes are adjacent, and we can normalize the root system so that either orbit is the short orbit. Hence both orbits have tight Bruhat orders.

The case $W=H_{3}$. The Bruhat orderings of the $H_{3}$-orbits of $\omega_{1}$ and $\omega_{3}$ are displayed in Figure 3, with the covering edges generated by the $i$-th simple reflection labeled $i$. The non-minimal vertices corresponding to $\Phi_{\langle i\rangle}$-dominant points are those that are the upper endpoint of an edge labeled $i$, and no other labeled edge. It is easy to check that these sets of vertices form chains, so Theorem 2.3 implies that the two orders are tight.

The case $W=B_{n}, J^{c}=\left\{s_{2}\right\}$. In the standard realization, $B_{n}$ acts as the group of signed permutations of the coordinates in $V=\mathbf{R}^{n}$, and the dominant chamber may be taken to consist of vectors with weakly increasing, nonnegative coordinates. With these choices, the vector $\theta=(0,1, \ldots, 1)$ is dominant with stabilizer $W_{\langle 2\rangle}$, and the vectors that are dominant with respect to $\Phi_{\langle i\rangle}$ are those $\mu=\left(a_{1}, \ldots, a_{n}\right)$ such that $0 \leqslant a_{1} \leqslant \cdots \leqslant a_{i-1}$ and $a_{i} \leqslant a_{i+1} \leqslant \cdots \leqslant a_{n}$. Hence,

$$
\left(B_{n} \theta\right)_{\langle i\rangle}=\left\{\begin{array}{cc}
\left\{\mu_{0}, \mu_{1}, \ldots, \mu_{n-i}, \nu_{0}, \nu_{1}, \ldots, \nu_{n-i+1}\right\} & \text { if } i>1 \\
\left\{\mu_{0}, \mu_{1}, \ldots, \mu_{n-1}\right\} & \text { if } i=1
\end{array}\right.
$$

where $\mu_{j}=\left(1^{i-1}(-1)^{j} 01^{n-i-j}\right)$ and $\nu_{j}=\left(01^{i-2}(-1)^{j} 1^{n-j-i+1}\right)$, using the notation $a^{k}$ to denote a string of $k$ a's. Noting that $\nu_{j}-\mu_{j}, \mu_{j}-\nu_{j+1}$, and $\mu_{j}-\mu_{j+1}$ are each positive multiples of positive roots, it follows via Remark 1.2(b) that

$$
\begin{array}{cc}
\nu_{0}<_{\mathrm{B}} \mu_{0}<_{\mathrm{B}} \nu_{1}<_{\mathrm{B}} \mu_{1}<_{\mathrm{B}} \cdots<_{\mathrm{B}} \nu_{n-i+1} & (i>1), \\
\mu_{0}<_{\mathrm{B}} \mu_{1}<_{\mathrm{B}} \cdots<_{\mathrm{B}} \mu_{n-1} & (i=1) .
\end{array}
$$

Thus Theorem 2.3 implies that $\left(B_{n} \theta,<_{\mathrm{B}}\right)$ is tight. 

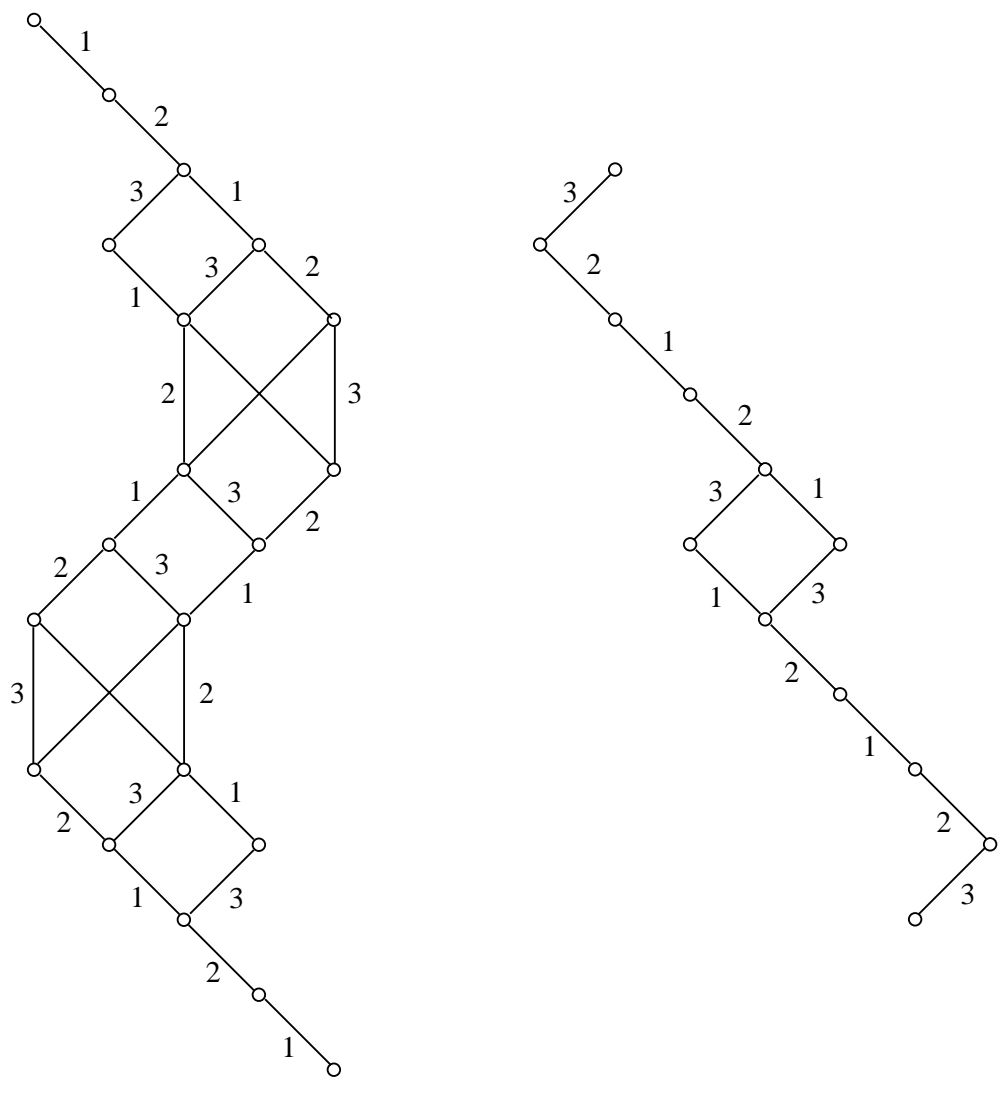

Figure 3: The Bruhat orderings of $H_{3} \omega_{1}$ and $H_{3} \omega_{3}$.

The remaining listed cases involve quotients by non-maximal parabolic subgroups $W_{J}$ with $J^{c}=\left\{s_{j}, s_{j+1}\right\}$ (for $W=A_{n}$ ) and $J^{c}=\left\{s_{1}, s_{2}\right\}$ (for $W=B_{n}$ ). By Lemma 3.3, one may deduce that these quotients are tight by verifying that the corresponding face of the dominant chamber is confined to a single face of $\mathcal{H}$. We omit the proof, since a stronger result for affine Weyl groups will be given later in the proof of Theorem 5.12.

Turning to the converse, we seek to show that the Bruhat orderings of all remaining quotients are not tight. For the cases with $\left|J^{c}\right|=1$, our strategy is to show that the corresponding orbits fail the stratification test in Proposition 3.1. Once this is complete, Lemmas 3.3 and 3.4 combine to eliminate the cases with $\left|J^{c}\right|>1$.

For the former, note that the following fundamental weights are roots that include an orthogonal pair of simple roots in their orbits: $\omega_{n-1}$ in $B_{n}$ and $D_{n}$ ( $n \geqslant 4$ only), $\omega_{2}$ in $H_{3}$ and $E_{6}, \omega_{1}$ in $E_{7}, \omega_{8}$ in $E_{8}$, and $\omega_{4}$ in $H_{4}$. It follows via Lemma 3.5 that the Bruhat orderings of their orbits fail the stratification test. More generally, if the $j$-th fundamental weight for $W$ generates an orbit that fails the stratification test, then Lemma 3.6 implies that the same is true for the $j$-th fundamental weight for any finite Coxeter group that includes $W$ as a parabolic subgroup. In this way one may deduce that all remaining orbits $W \omega_{j}$ fail the stratification test except possibly $F_{4} \omega_{2}, F_{4} \omega_{3}$, $H_{4} \omega_{1}$, and $H_{4} \omega_{3}$. 
The cases $W \omega_{j}=F_{4} \omega_{2}$ and $F_{4} \omega_{3}$. If we normalize the root system so that $\Phi$ is crystallographic and $\alpha_{1}$ and $\alpha_{2}$ are short, then an easy calculation shows that

$$
\begin{aligned}
& s_{2} s_{1} s_{3} s_{2} s_{3} \omega_{3}=\omega_{3}-2 \alpha_{1}-4 \alpha_{2}-2 \alpha_{3}=: \mu, \\
& s_{2} s_{3} s_{2} s_{4} s_{3} \omega_{3}=\omega_{3}-4 \alpha_{2}-3 \alpha_{3}-\alpha_{4}=: \nu .
\end{aligned}
$$

In particular, the coefficient of $\alpha_{2}$ in $\mu-\nu$ is zero. On the other hand, $W_{\langle 2\rangle}$ is the direct product of the subgroups generated by $\left\{s_{1}\right\}$ and $\left\{s_{3}, s_{4}\right\}$, so if $\mu$ and $\nu$ were in the same $W_{\langle 2\rangle}$-orbit, then the coefficient of $\alpha_{1}$ in either $\mu-\nu$ or $\mu-s_{1} \nu=\mu-\nu+4 \alpha_{1}$ (i.e., \pm 2 ) would have to be zero. Thus the $W_{\langle 2\rangle}$-orbits in $W \omega_{3}$ are not stratified, and hence by symmetry (Lemma 3.7), the same is true for the $W_{\langle 3\rangle}$-orbits in $W \omega_{2}$.

The cases $W \omega_{j}=H_{4} \omega_{1}$ and $H_{4} \omega_{3}$. Another easy calculation reveals that

$$
\begin{aligned}
s_{3} s_{2} s_{1} s_{2} s_{3} s_{1} s_{2} s_{1} \omega_{1} & =\omega_{1}-(2+2 r) \alpha_{1}-(1+3 r) \alpha_{2}-(1+2 r) \alpha_{3}=: \mu, \\
s_{3} s_{2} s_{1} s_{4} s_{3} s_{2} s_{1} \omega_{1} & =\omega_{1}-(1+r) \alpha_{1}-(1+2 r) \alpha_{2}-(1+2 r) \alpha_{3}-r \alpha_{4}=: \nu,
\end{aligned}
$$

where $r=-\left\langle\alpha_{1}, \alpha_{2}^{\vee}\right\rangle=(1+\sqrt{5}) / 2$ denotes the golden ratio. In particular, the coefficient of $\alpha_{3}$ in $\mu-\nu$ is zero. On the other hand, $W_{\langle 3\rangle}$ is the direct product of the subgroups generated by $\left\{s_{1}, s_{2}\right\}$ and $\left\{s_{4}\right\}$, so if $\mu$ and $\nu$ were in the same $W_{\langle 3\rangle}$-orbit, then the coefficient of $\alpha_{4}$ in either $\mu-\nu$ or $s_{4} \mu-\nu=\mu-\nu-(1+2 r) \alpha_{4}$ (i.e., $r$ or $-(1+r)$ ) would have to be zero. Thus the $W_{\langle 3\rangle}$-orbits in $W \omega_{1}$ are not stratified, and hence by symmetry (Lemma 3.7), the same is true for the $W_{\langle 1\rangle}$-orbits in $W \omega_{3}$.

Bearing in mind that the $W$-orbit of a fundamental weight is generated by some irreducible component of $W$, a corollary of the above proof is that whenever $\left(W \omega_{j},<_{\mathrm{B}}\right)$ is not tight, there is some index $i$ such that the $W_{\langle i\rangle}$-orbits in $W \omega_{j}$ are not stratified. Thus we obtain the following partial converse to Proposition 3.1. It would be interesting to have a conceptual argument for it that avoids using the classification.

Theorem 3.9. A fundamental orbit $\left(W \omega_{j},<_{\mathrm{B}}\right)$ is tight if and only if the $W_{\langle i\rangle}$-orbits in $W \omega_{j}$ are stratified for all $i$.

Combining the above result with Lemma 3.3 yields a characterization of (finite) tight quotients that involves confining a face of the dominant chamber to a face of the double weight arrangement. However, face-confinement is also related to orbit stratification, so this leads to yet another characterization of tightness.

Corollary 3.10. The Bruhat ordering of $W^{J}$ is tight if and only if the $W_{\langle i\rangle}$-orbits in $W \theta$ are stratified for all $i$ and all dominant $\theta$ fixed by $W_{J}$.

Proof. If $\theta \in V$ is dominant and fixed by $W_{J}$, then the stabilizer of $\theta$ is some parabolic subgroup $W_{I}$ such that $J \subseteq I$. Hence ${ }^{\langle i\rangle} W^{J} \supseteq{ }^{\langle i\rangle} W^{I}$ for all $i$, and it follows via Theorem 2.3 that if $\left(W^{J},<_{\mathrm{B}}\right)$ is tight, then the same must be true for $\left(W \theta,<_{\mathrm{B}}\right)$. In particular, the $W_{\langle i\rangle}$-orbits in $W \theta$ must be stratified (Proposition 3.1).

Conversely, assume that the stated condition holds but that $\left(W^{J},<_{\mathrm{B}}\right)$ fails to be tight. Since $W_{J}$ fixes each fundamental weight $\omega_{j}$ such that $s_{j} \in S-J$, Theorem 3.9 
implies that each orbit $\left(W \omega_{j},<_{\mathrm{B}}\right)$ must be tight. It then follows via Lemma 3.3 that the set of dominant vectors with stabilizer $W_{J}$ is not confined to a single face of the double weight arrangement. Hence, there must be a hyperplane $H \in \mathcal{H}$ and a dominant pair $\theta, \theta^{\prime} \in V$ with stabilizer $W_{J}$ such that $H$ includes $\theta$ but not $\theta^{\prime}$. Selecting a normal vector for $H$, there must be some $x, y \in W$ and some $i$ such that $\left\langle\theta, x \omega_{i}\right\rangle=\left\langle\theta, y \omega_{i}\right\rangle$, and hence $\mu=x^{-1} \theta$ and $\nu=y^{-1} \theta$ are two points in $W \theta$ such that the coefficient of $\alpha_{i}^{\vee}$ in $\mu-\nu$ is zero. Given that the $W_{\langle i\rangle}$-orbits in $W \theta$ are stratified, this forces $\mu$ and $\nu$ into the same $W_{\langle i\rangle}$-orbit. However in that case, $\mu^{\prime}=x^{-1} \theta^{\prime}$ and $\nu^{\prime}=y^{-1} \theta^{\prime}$ must also be in the same $W_{\langle i\rangle}$-orbit, and this contradicts the choice of $H$.

REMARK 3.11. (a) One sees from the classification that the (irreducible) finite Weyl groups $W$ such that every quotient $\left(W^{\langle j\rangle},<_{\mathrm{B}}\right)$ is tight are those of rank $\leqslant 3$ and the symmetric groups. This is roughly equivalent to a result of Deodhar [D2].

(b) On a case-by-case basis (aided by machine computations), we have checked that the $W_{\langle i\rangle}$-orbits in $W \omega_{j}$ are stratified if and only if $\left(\left(W \omega_{j}\right)_{\langle i\rangle},<_{\mathrm{B}}\right)$ is a chain. Bearing in mind Theorem 2.3, this may be viewed as a sharper version of Theorem 3.9.

(c) If $W_{J}$ is the stabilizer of a minuscule weight, then the longest element $w_{J} \in W^{J}$ is fully commutative in the sense of $[\mathbf{S 1}]$. However, there exist other quotients whose longest elements are fully commutative, such as $H_{3}^{\langle 3\rangle}$, and it is possible to show directly (without the classification) that this property implies tightness. Indeed, given that $w_{J}$ is fully commutative, one knows that $\left(W^{J},<_{\mathrm{B}}\right)$ is a distributive lattice (Theorems 3.2 and 7.1 of $[\mathbf{S 1}]$ ). Furthermore, one may construct the subposet of join-irreducible elements by building the heap of any reduced expression for $w_{J}$. This subposet includes the non-identity elements of ${ }^{\langle i\rangle} W^{J}$, and there is a bijection between these elements and the occurrences of $s_{i}$ in the reduced expression. The rules of construction show that these elements are totally ordered in the heap (for details, see [S1]), and thus a fully commutative quotient fits the tightness criterion of Theorem 2.3.

\section{Affine Weyl groups and their quotients.}

Now we turn to the Coxeter groups of affine type; these are the Weyl groups of affine Kac-Moody algebras. As with the finite Weyl groups, there is a geometric meaning associated with the Bruhat order - there are analogues of the flag variety for affine KacMoody groups, these varieties have cell decompositions indexed by the Weyl group, and the relation of inclusion of cell closures is the Bruhat order. (For details, see $[\mathbf{K}]$.)

In this section, $\Phi$ shall denote a finite crystallographic root system (i.e., $\left\langle\alpha, \beta^{\vee}\right\rangle \in \mathbf{Z}$ for all $\alpha, \beta \in \Phi)$, and $W$ the corresponding finite Coxeter group. In this situation, the roots and co-roots both generate lattices, denoted $\mathbf{Z} \Phi$ and $\mathbf{Z} \Phi^{\vee}$, respectively. As in the previous section, we let $\alpha_{1}, \ldots, \alpha_{n}$ denote simple roots, $s_{1}, \ldots, s_{n}$ the corresponding simple reflections, and $\omega_{1}, \ldots, \omega_{n}$ the fundamental weights. We may also assume that $\langle$,$\rangle is positive definite on V$, the ambient space for $\Phi$.

\section{A. A linear-affine dictionary.}

The affine Weyl group $\widetilde{W}$ associated to $\Phi$ is typically represented as a group of affine transformations of $V$. On the other hand, affine Weyl groups are also Coxeter groups, and thus are also representable as groups of linear transformations generated 
by reflections through central hyperplanes (in non-Euclidean spaces). These two points of view will be important in our analysis, so we begin with an explicit description of their relationship; we have not seen this particular approach used elsewhere.

Assuming for initial simplicity that $\Phi$ is irreducible, let us introduce the space

$$
\widetilde{V}:=V \oplus \mathbf{R} \delta \oplus \mathbf{R} \delta^{\prime}
$$

where $\delta$ and $\delta^{\prime}$ denote two new coordinates. Extend the bilinear form to $\widetilde{V}$ by defining

$$
\left\langle a \delta+a^{\prime} \delta^{\prime}+\mu, b \delta+b^{\prime} \delta^{\prime}+\nu\right\rangle=a b^{\prime}+a^{\prime} b+\langle\mu, \nu\rangle \quad(\mu, \nu \in V)
$$

for all scalars $a, b, a^{\prime}, b^{\prime}$. Note that $\delta$ and $\delta^{\prime}$ are both orthogonal to $V$, and that $\langle$,$\rangle is$ nondegenerate on $\widetilde{V}$ and positive semidefinite on $V \oplus \mathbf{R} \delta$ and $V \oplus \mathbf{R} \delta^{\prime}$.

We define the affine root system associated to $\Phi$ to be

$$
\widetilde{\Phi}:=\{k \delta+\beta: k \in \mathbf{Z}, \beta \in \Phi\} \subset \widetilde{V} .
$$

As a set of simple roots for $\widetilde{\Phi}$, we use the simple roots $\alpha_{1}, \ldots, \alpha_{n}$ for $\Phi$, together with

$$
\alpha_{0}:=\delta-\widetilde{\alpha}
$$

where $\widetilde{\alpha} \in \Phi$ denotes the highest root (i.e., the unique root in $\Phi$ that is maximal with respect to the standard order). In these terms, the positive roots consist of those roots $k \delta+\beta$ such that either $k>0$, or $k=0$ and $\beta \in \Phi^{+}$.

The affine Weyl group $\widetilde{W}$ corresponding to $\Phi$ may be defined as the subgroup of $G L(\widetilde{V})$ generated by $\widetilde{S}:=\left\{s_{0}, \ldots, s_{n}\right\}$, where $s_{i}$ now denotes reflection through the hyperplane in $\widetilde{V}$ orthogonal to $\alpha_{i}$. To show that this construction yields a legitimate Coxeter system with root system $\widetilde{\Phi}$ amounts to checking that (i) the angle between $\alpha_{i}$ and $\alpha_{j}$ is $\pi\left(1-1 / m_{i j}\right)$, where $\left[m_{i j}\right]$ is some Coxeter matrix, and (ii) every root $k \delta+\beta$ is in the $\widetilde{W}$-orbit of some simple root. The former is easy and the latter is a straightforward induction with respect to $k$.

Turning to the representation of $\widetilde{W}$ as a group of affine transformations of $V$, let us define a linear transformation $t_{\mu}: \widetilde{V} \rightarrow \widetilde{V}$ for each $\mu \in V$ by setting

$$
t_{\mu}\left(\delta^{\prime}\right)=\delta^{\prime}-\frac{1}{2}\|\mu\|^{2} \delta+\mu, \quad t_{\mu}(\delta)=\delta, \quad t_{\mu}(\theta)=\theta-\langle\theta, \mu\rangle \delta \quad(\theta \in V),
$$

where $\|\mu\|:=\sqrt{\langle\mu, \mu\rangle}$ denotes the usual Euclidean norm. Clearly $t_{0}$ is the identity map.

Proposition 4.1. We have

(a) $t_{\mu} t_{\nu}=t_{\mu+\nu}$ for all $\mu, \nu \in V$.

(b) $s_{k \delta+\beta}=s_{\beta} t_{k \beta} \vee=t_{-k \beta} \vee s_{\beta}$ for all roots $k \delta+\beta \in \widetilde{\Phi}$.

(c) $T\left(\Phi^{\vee}\right):=\left\{t_{\mu}: \mu \in \mathbf{Z} \Phi^{\vee}\right\}$ is an abelian subgroup of $\widetilde{W}$.

(d) $s_{\beta} t_{\mu}=t_{s_{\beta}(\mu)} s_{\beta}$ for all $\beta \in \Phi$ and $\mu \in V$.

(e) $\widetilde{W}$ is the semi-direct product $T\left(\Phi^{\vee}\right) \rtimes W$. 
Proof. (a) For $\theta \in V$, we have

$$
t_{\mu} t_{\nu}(\theta)=t_{\mu}(\theta-\langle\theta, \nu\rangle \delta)=\theta-\langle\theta, \mu\rangle \delta-\langle\theta, \nu\rangle \delta=t_{\mu+\nu}(\theta) .
$$

It is also clear that $t_{\mu} t_{\nu}(\delta)=t_{\mu+\nu}(\delta)=\delta$, and

$$
\begin{aligned}
t_{\mu} t_{\nu}\left(\delta^{\prime}\right) & =t_{\mu}\left(\delta^{\prime}-\frac{1}{2}\|\nu\|^{2} \delta+\nu\right)=\delta^{\prime}-\frac{1}{2}\|\mu\|^{2} \delta-\frac{1}{2}\|\nu\|^{2} \delta-\langle\mu, \nu\rangle \delta+\mu+\nu \\
& =\delta^{\prime}-\frac{1}{2}\|\mu+\nu\|^{2} \delta+(\mu+\nu)=t_{\mu+\nu}\left(\delta^{\prime}\right) .
\end{aligned}
$$

(b) For $\theta \in V$, we have

$$
s_{k \delta+\beta}(\theta)=\theta-\left\langle\theta, \beta^{\vee}\right\rangle(k \delta+\beta)=s_{\beta}(\theta)-k\left\langle\theta, \beta^{\vee}\right\rangle \delta=s_{\beta} t_{k \beta \vee}(\theta)=t_{-k \beta} s_{\beta}(\theta) .
$$

Also, every reflection and every operator $t_{\mu}$ acts trivially on $\delta$, whereas

$$
\begin{aligned}
s_{k \delta+\beta}\left(\delta^{\prime}\right) & =\delta^{\prime}-\left\langle\delta^{\prime}, k \delta+\beta\right\rangle(k \delta+\beta)^{\vee} \\
& =\delta^{\prime}-\frac{1}{2}\left\|k \beta^{\vee}\right\|^{2} \delta-k \beta^{\vee}=s_{\beta} t_{k \beta^{\vee}}\left(\delta^{\prime}\right)=t_{-k \beta^{\vee}} s_{\beta}\left(\delta^{\prime}\right) .
\end{aligned}
$$

(c) The fact that $T\left(\Phi^{\vee}\right)$ is an abelian group is a corollary of (a). Moreover, (b) implies that for each $\beta \in \Phi$, we have $t_{\beta \vee}=s_{\beta} s_{\delta+\beta} \in \widetilde{W}$.

(d) For $\theta \in V$, we have

$$
s_{\beta} t_{\mu} s_{\beta}(\theta)=s_{\beta}\left(s_{\beta}(\theta)-\left\langle s_{\beta}(\theta), \mu\right\rangle \delta\right)=\theta-\left\langle\theta, s_{\beta}(\mu)\right\rangle \delta=t_{s_{\beta}(\mu)}(\theta),
$$

whereas for $\delta^{\prime}$, we have

$$
s_{\beta} t_{\mu} s_{\beta}\left(\delta^{\prime}\right)=s_{\beta}\left(\delta^{\prime}-\frac{1}{2}\|\mu\|^{2} \delta+\mu\right)=\delta^{\prime}-\frac{1}{2}\|\mu\|^{2} \delta+s_{\beta}(\mu)=t_{s_{\beta}(\mu)}\left(\delta^{\prime}\right) .
$$

(e) From (b), it follows that $\widetilde{W}$ is generated by $T\left(\Phi^{\vee}\right)$ and the reflections $s_{\beta} \in W$. Furthermore, (d) implies that $T\left(\Phi^{\vee}\right)$ is a normal subgroup, and it intersects $W$ trivially since $t_{\mu}$ fixes $\delta^{\prime}$ only if $\mu=0$, whereas every $w \in W$ has this property.

In the following, it will be convenient to introduce the notation

$$
x(\mu):=t_{\mu}\left(\delta^{\prime}\right)=\delta^{\prime}-\frac{1}{2}\|\mu\|^{2} \delta+\mu \quad(\mu \in V) .
$$

Corollary 4.2. The cross section $X=\{x(\mu): \mu \in V\}$ is $\widetilde{W}$-stable. Moreover,

(a) $t_{\mu}(x(\nu))=x(\mu+\nu)$ for all $\mu, \nu \in V$, and

(b) $s_{\beta}(x(\nu))=x\left(s_{\beta}(\nu)\right)$ for all $\beta \in \Phi$ and $\nu \in V$.

Proof. We have $t_{\mu}(x(\nu))=t_{\mu} t_{\nu}\left(\delta^{\prime}\right)=t_{\mu+\nu}\left(\delta^{\prime}\right)=x(\mu+\nu)$ by Proposition 4.1(a) and $s_{\beta}(x(\mu))=s_{\beta} t_{\mu}\left(\delta^{\prime}\right)=t_{s_{\beta}(\mu)} s_{\beta}\left(\delta^{\prime}\right)=x\left(s_{\beta}(\mu)\right)$ by Proposition 4.1(d). 
Thus under the identification $x(\mu) \leftrightarrow \mu$, the action of $\widetilde{W}$ on the space $X$ may be transported to an action on $V$; in this transported form, $\widetilde{W}$ acts as a group of affine transformations of $V$ in which $t_{\mu}$ acts as translation by $\mu$ and $W$ acts in the usual (linear) way. Note also that the induced action of $s_{k \delta-\beta}$ on $V$ is a reflection through the affine hyperplane $\{\mu \in V:\langle\mu, \beta\rangle=k\}$.

REMARK 4.3. (a) Associated to the reflection representation of any Coxeter group is the Tits cone - the union of the orbits of all dominant vectors, or equivalently, the set of points on the negative side of finitely many of the root hyperplanes. It is a proper subset of the ambient space if and only if the Coxeter group is infinite. In the case of an affine Weyl group as above, it is not hard to check that the Tits cone consists of the line $\mathbf{R} \delta$ together with all points whose $\delta^{\prime}$-coordinate is positive. After discarding the (trivial) orbits in $\mathbf{R} \delta$, it follows that all remaining orbits belong to spaces of the form $a X+b \delta$, where $a$ and $b$ are scalars and $a>0$. Thus for the purpose of comparing the Bruhat and standard orderings of orbits of dominant vectors, there is no loss of generality in restricting our attention to the orbits in $X$.

(b) Note that $\langle x(\mu), k \delta+\beta\rangle=k+\langle\mu, \beta\rangle$, so the dominant part of $X$ consists of those $x(\theta)$ with $\theta$ in the fundamental alcove; i.e., the simplex ${ }^{34}$

$$
A_{0}:=\left\{\theta \in V:\langle\theta, \widetilde{\alpha}\rangle \leqslant 1,\left\langle\theta, \alpha_{i}\right\rangle \geqslant 0, i=1, \ldots, n\right\} .
$$

We let $\theta_{0}, \theta_{1}, \ldots, \theta_{n}$ denote the vertices of $A_{0}$, labeled so that $\theta_{0}=0,\left\langle\theta_{i}, \widetilde{\alpha}\right\rangle=1(i \neq 0)$, and $\left\langle\theta_{i}, \alpha_{j}\right\rangle=0$ for $i \neq j$. Note that every parabolic subgroup of $\widetilde{W}$, except $\widetilde{W}$ itself, occurs as the stabilizer of some point $x(\theta)$ for $\theta \in A_{0}$.

The preceding discussion is more tedious without the hypothesis that $\Phi$ is irreducible. If $\Phi_{1}, \ldots, \Phi_{l}$ are the irreducible components of $\Phi$, let $V_{i}=\operatorname{Span} \Phi_{i}$ and define $V_{0}$ to be the orthogonal complement of $\Phi$ in $V$, so that $V=V_{0} \oplus \cdots \oplus V_{l}$. We then use

$$
\widetilde{V}:=V \oplus \mathbf{R} \delta_{0} \oplus \mathbf{R} \delta_{0}^{\prime} \oplus \cdots \oplus \mathbf{R} \delta_{l} \oplus \mathbf{R} \delta_{l}^{\prime}
$$

as the ambient space, where $\delta_{i}$ and $\delta_{i}^{\prime}$ are orthogonal to $V,\left\langle\delta_{i}, \delta_{i}^{\prime}\right\rangle=\left\langle\delta_{i}^{\prime}, \delta_{i}\right\rangle=1$, and all other pairings among $\delta_{0}, \delta_{0}^{\prime}, \ldots, \delta_{l}, \delta_{l}^{\prime}$ are zero. The associated affine root system is

$$
\widetilde{\Phi}:=\bigcup_{1 \leqslant i \leqslant l}\left\{k \delta_{i}+\beta: k \in \mathbf{Z}, \beta \in \Phi_{i}\right\},
$$

and the simple roots are those of $\Phi$, together with $\delta_{i}-\widetilde{\alpha}_{i}(i=1, \ldots, l)$, where $\widetilde{\alpha}_{i}$ denotes the highest root of $\Phi_{i}$.

The linear transformations $t_{\mu}: \widetilde{V} \rightarrow \widetilde{V}$ may be defined for $\mu \in V$ by setting

$$
t_{\mu}\left(\delta_{i}^{\prime}\right)=\delta_{i}^{\prime}-\frac{1}{2}\left\|\mu_{i}\right\|^{2} \delta_{i}+\mu_{i}, \quad t_{\mu}\left(\delta_{i}\right)=\delta_{i}, \quad t_{\mu}(\theta)=\theta-\sum\left\langle\theta, \mu_{i}\right\rangle \delta_{i} \quad(\theta \in V),
$$

\footnotetext{
${ }^{3}$ The reader will not be confused by the use of $A_{0}$ to denote an alcove and $A_{n}$ a Coxeter group.

${ }^{4}$ It is more accurate to say that $A_{0} \cap \operatorname{Span} \Phi$ is a simplex. Similarly, the vertices $\theta_{0}, \ldots, \theta_{n}$ are not well-defined unless we add the requirement that $\theta_{i} \in \operatorname{Span} \Phi$.
} 
where $\mu_{i}$ denotes the orthogonal projection of $\mu$ onto $V_{i}$. In this form, Proposition 4.1 remains valid as stated (except that the roots appearing in (b) take the form $k \delta_{i}+\beta$ for some $i>0$ ), although the proofs require slightly more calculation. Also, the appropriate generalization of the cross section $X=\{x(\mu): \mu \in V\}$ is obtained by setting

$$
x(\mu):=t_{\mu}\left(\delta^{\prime}\right)=\delta^{\prime}-\frac{1}{2}\left(\left\|\mu_{0}\right\|^{2} \delta_{0}+\cdots+\left\|\mu_{l}\right\|^{2} \delta_{l}\right)+\mu,
$$

where $\delta^{\prime}:=\delta_{0}^{\prime}+\cdots+\delta_{l}^{\prime}$. In this form, Corollary 4.2 holds verbatim. Note also that $x(\theta)$ is still dominant if and only if $\theta$ is in the fundamental alcove

$$
A_{0}:=\left\{\theta \in V: 0 \leqslant\langle\theta, \beta\rangle \leqslant 1 \text { for all } \beta \in \Phi^{+}\right\} .
$$

However, $A_{0} \cap \operatorname{Span} \Phi$ is now a product of simplices.

\section{B. The Bruhat ordering of affine orbits.}

In this subsection, our goal is to describe more explicitly the Bruhat orderings of the $\widetilde{W}$-orbits of dominant points in $\widetilde{V}$. As noted in Remark 4.3(a), there is no loss of generality in restricting our attention to the orbits in $X$, so we may assume throughout that the dominant generator of the orbit is a point of the form $x(\theta)$ for some $\theta \in A_{0}$. Note also that since $\widetilde{W}$ is the semi-direct product of $W$ and the translation group $T\left(\Phi^{\vee}\right)$, the orbit of $x(\theta)$ consists of all points $x(\mu)$ such that $\mu \in W \theta+\mathbf{Z} \Phi^{\vee}$.

Proposition 4.4. For all $\mu \in W \theta+\mathbf{Z} \Phi^{\vee}$, the rank of $x(\mu)$ in $\left(\widetilde{W} x(\theta),<_{\mathrm{B}}\right)$ is

$$
r(x(\mu))=\sum_{\beta \in \Phi^{+}}\left\lceil\left\langle\mu^{+}, \beta\right\rangle\right\rceil-\#\left\{\beta \in \Phi^{+}:\langle\mu, \beta\rangle>0\right\},
$$

where $\mu^{+}$denotes the $\Phi$-dominant element in the $W$-orbit of $\mu$.

Proof. The rank of $x(\mu)$ is the number of root hyperplanes that separate $x(\theta)$ from $x(\mu)$ (see Remark 1.2(c)). Thus for each $\beta \in \Phi^{+}$, we need to count the number of integers $k \geqslant 0$ such that $\langle x(\mu), k \delta+\beta\rangle=k+\langle\mu, \beta\rangle<0$, as well as the number of positive $k$ such that $\langle x(\mu), k \delta-\beta\rangle=k-\langle\mu, \beta\rangle<0$. If we temporarily increase this count by 1 if $\langle\mu, \beta\rangle>0$, this is the number of integers $k$ such that $0 \leqslant k<\mid\langle\mu$, $\beta\rangle \mid$; i.e., $\lceil|\langle\mu, \beta\rangle|\rceil$. Hence,

$$
r(x(\mu))=\sum_{\beta \in \Phi^{+}}\lceil|\langle\mu, \beta\rangle|\rceil-\#\left\{\beta \in \Phi^{+}:\langle\mu, \beta\rangle>0\right\} .
$$

This may be seen as equivalent to the claimed result upon recognizing that the multiset of scalars $|\langle\mu, \beta\rangle|$, as $\beta$ varies over $\Phi^{+}$, is constant on the $W$-orbit of $\mu$.

LEMMA 4.5. In the $\widetilde{W}$-orbit of $x(\theta)$, we have the relations

$$
\begin{array}{ll}
x(\mu)<_{\mathrm{B}} x\left(\mu+\beta^{\vee}\right) & \text { if }\langle\mu, \beta\rangle \geqslant 0, \beta \in \Phi^{+}, \\
x(\mu)<_{\mathrm{B}} x\left(\mu-\beta^{\vee}\right) & \text { if }\langle\mu, \beta\rangle \leqslant 1, \beta \in \Phi^{+} .
\end{array}
$$


Proof. If $\beta \in \Phi^{+}$and $\langle\mu, \beta\rangle \geqslant 0$, then $\left\langle s_{\beta}(x(\mu)), \delta-\beta\right\rangle=1+\langle\mu, \beta\rangle>0$, whence

$$
x(\mu) \leqslant_{\mathrm{B}} s_{\beta} x(\mu)<_{\mathrm{B}} s_{\delta-\beta} s_{\beta} x(\mu)=t_{\beta^{\vee}} x(\mu)=x\left(\mu+\beta^{\vee}\right) .
$$

Similarly, if $\langle\mu, \beta\rangle \leqslant 1$, then $\langle x(\mu), \delta-\beta\rangle \geqslant 0$ and

$$
\left\langle s_{\delta-\beta}(x(\mu)), \beta\right\rangle=\langle x(\mu), 2 \delta-\beta\rangle=2-\langle\mu, \beta\rangle>0
$$

so we have $x(\mu) \leqslant_{\mathrm{B}} s_{\delta-\beta} x(\mu)<_{\mathrm{B}} s_{\beta} s_{\delta-\beta} x(\mu)=t_{-\beta} \vee x(\mu)=x\left(\mu-\beta^{\vee}\right)$.

Let $c^{+}$denote the smallest integer $>c$ and $c^{-}$the largest integer $<c$. If $c$ is an integer, then $c^{+}=c+1$ and $c^{-}=c-1$, so these are not the "ceiling" and "floor" of $c$.

Proposition 4.6. The Bruhat ordering of the $\widetilde{W}$-orbit of $x(\theta)$ is generated by the transitive closure of the relations

$$
\begin{array}{cl}
x(\mu)<_{\mathrm{B}} x\left(\mu+\left(c^{+}-c\right) \beta^{\vee}\right) & \text { if }\langle\mu, \beta\rangle=c \geqslant 0, \beta \in \Phi^{+}, \\
x(\mu)<_{\mathrm{B}} s_{\beta} x(\mu)=x\left(\mu-c \beta^{\vee}\right) & \text { if }\langle\mu, \beta\rangle=c>1, \beta \in \Phi^{+}, \\
x(\mu)<_{\mathrm{B}} x\left(\mu-\left(c-c^{-}\right) \beta^{\vee}\right) & \text { if }\langle\mu, \beta\rangle=c \leqslant 1, \beta \in \Phi^{+}, \\
x(\mu)<_{\mathrm{B}} s_{\delta-\beta} x(\mu)=x\left(\mu+(1-c) \beta^{\vee}\right) & \text { if }\langle\mu, \beta\rangle=c<0, \beta \in \Phi^{+} .
\end{array}
$$

Furthermore, the Bruhat ordering of each double quotient $(\widetilde{W} x(\theta))_{I}$ is generated by the transitive closure of all such relations that involve pairs of elements from $(\widetilde{W} x(\theta))_{I}$.

Proof. We know that the Bruhat ordering of any orbit or its $\widetilde{\Phi}_{I}$-dominant part is generated by reflections (Proposition $1.5(\mathrm{c})$ ). Thus given a point $x(\mu)$ in $\widetilde{W} x(\theta)$, it suffices to identify a subset of the reflection images of $x(\mu)$ large enough to include all of those that cover $x(\mu)$.

Given $\beta \in \Phi^{+}$, set $\langle\mu, \beta\rangle=c$. Assuming $k \in \mathbf{Z}^{\geqslant 0}$ (so that $k \delta+\beta$ is a positive root), we have $\langle x(\mu), k \delta+\beta\rangle=k+c$, and hence

$$
x(\mu)<_{\mathrm{B}} s_{k \delta+\beta} x(\mu)=x\left(\mu-(k+c) \beta^{\vee}\right)
$$

if and only if $k+c>0$. The minimal choice for $k$ is either $(-c)^{+}=-c^{-}$(if $c \leqslant 1$ ) or 0 (if $c>1$ ); these choices yield the relations (4.1c) and (4.1b). Furthermore, given the conditions $k \geqslant 0$ and $k+c>0$, we have $\left\langle x\left(\mu-(k+c) \beta^{\vee}\right), \beta\right\rangle=-2 k-c<0$, and hence

$$
x\left(\mu-(k+c) \beta^{\vee}\right)<_{\mathrm{B}} x\left(\mu-(k+1+c) \beta^{\vee}\right)
$$

by Lemma 4.5. Thus (4.2) is a covering relation only if $k$ is minimal. This also holds in the Bruhat ordering of $(\widetilde{W} x(\theta))_{I}$ by convexity; indeed, if $x(\mu)$ and $x\left(\mu-(k+c) \beta^{\vee}\right)$ are both $\widetilde{\Phi}_{I}$-dominant, then the same is true for $x\left(\mu-t \beta^{\vee}\right)$ for $0 \leqslant t \leqslant k+c$, and thus only the minimal choices for $k$ could yield covering relations of $\left((\widetilde{W} x(\theta))_{I},<_{\mathrm{B}}\right)$. 
Similarly, if $k \in \mathbf{Z}^{>0}$ (so $k \delta-\beta$ is a positive root), then $\langle x(\mu), k \delta-\beta\rangle=k-c$ and

$$
x(\mu)<_{\mathrm{B}} s_{k \delta-\beta} x(\mu)=x\left(\mu+(k-c) \beta^{\vee}\right)
$$

if and only if $k>c$. Here, the minimal choice for $k$ is either $c^{+}$(if $c \geqslant 0$ ) or 1 (if $c<0$ ), yielding (4.1a) and (4.1d). Furthermore, assuming $k>0$ and $k>c$, we have $\left\langle x\left(\mu+(k-c) \beta^{\vee}\right), \beta\right\rangle=2 k-c>1$, and hence Lemma 4.5 implies that (4.3) may be a covering relation only if $k$ is minimal. Again, convexity considerations allow us to conclude that the same is true in the Bruhat ordering of $(\widetilde{W} x(\theta))_{I}$.

Note that $W$ is the parabolic subgroup of $\widetilde{W}$ generated by $S$, and that the double quotient $(\widetilde{W} x(\theta))_{S}$ consists of all points $x(\mu)$ in the $\widetilde{W}$-orbit of $x(\theta)$ such that $\mu \in V$ is dominant with respect to $\Phi=\widetilde{\Phi}_{S}$. In this double quotient, we claim that only the relations in (4.1a) are needed to generate the Bruhat order. Indeed, for each covering relation $x(\mu)<_{\mathrm{B}} x(\nu)$ having the form of (4.1b), (4.1c), or (4.1d), either $\mu$ or $\nu$ fails to be $\Phi$-dominant. Thus,

COROLlary 4.7. The Bruhat ordering of the double quotient $(\widetilde{W} x(\theta))_{S}$ is generated by the relations $x(\mu)<_{\mathrm{B}} x(\nu)$, where $\mu, \nu \in W \theta+\mathbf{Z} \Phi^{\vee}$ are $\Phi$-dominant, and

$$
\nu-\mu=\left(c^{+}-c\right) \beta^{\vee} \quad\left(\langle\mu, \beta\rangle=c, \beta \in \Phi^{+}\right) .
$$

C. The (lack of) tight quotients in affine Weyl groups.

Note that the standard ordering of $\widetilde{V}$ generated by $\widetilde{\Phi}$ induces partial orderings on any subset of $\widetilde{V}$, including the cross section $X$ and the subspace $V$. Moreover, $V$ also carries a standard ordering relative to the root system $\Phi$; however, these two "standard" orderings of $V$ coincide: a point in $\operatorname{Span} \Phi$ is in the positive span of $\Phi^{+}$if and only if it is in the positive span of $\widetilde{\Phi}^{+}$.

For simplicity, let us assume that $W$ is irreducible, so that $\Phi$ has a highest root $\widetilde{\alpha}$.

LEMma 4.8. For all $\mu, \nu \in V$, we have $x(\mu) \succcurlyeq x(\nu)$ if and only if $\|\mu\| \leqslant\|\nu\|$ and $\nu-\mu \preccurlyeq c \widetilde{\alpha}$, where $c=\frac{1}{2}\left(\|\nu\|^{2}-\|\mu\|^{2}\right)$.

Proof. By definition, we have $x(\mu)-x(\nu)=(\mu-\nu)+c \delta$, with $c$ defined as above. The coefficient of $\alpha_{0}=\delta-\widetilde{\alpha}$ in this quantity is $c$, so $x(\mu)-x(\nu)$ is in the nonnegative span of the simple roots of $\widetilde{\Phi}$ if and only if $c \geqslant 0$ and $x(\mu)-x(\nu)-c \alpha_{0}=(\mu-\nu)+c \widetilde{\alpha}$ is in the nonnegative span of the simple roots of $\Phi$.

Unfortunately, the orbits generated by the (linear) reflection representations of affine Weyl groups do not afford any nontrivial tight double quotients.

THEOREM 4.9. If $W$ is irreducible and the Bruhat ordering of $(\widetilde{W} x(\theta))_{I}$ is tight for some $\theta \in A_{0}$, then $W$ is of rank 1 or $I=\widetilde{S}$.

Proof. It suffices to show that if $W$ is of rank $\geqslant 2$ and $I=\langle i\rangle=\widetilde{S}-\left\{s_{i}\right\}$, then the Bruhat ordering of $(\widetilde{W} x(\theta))_{I}$ cannot be tight. For this, we begin by noting that the portion of $X$ that is $\widetilde{\Phi}_{\langle i\rangle}$-dominant consists of all $x(\mu)$ such that $\left\langle\mu, \alpha_{j}\right\rangle \geqslant 0$ (for 
$j \neq i, 0$ ) and $\langle\mu, \widetilde{\alpha}\rangle \leqslant 1$ (if $i \neq 0$ ). If we replace the condition $\langle\mu, \widetilde{\alpha}\rangle \leqslant 1$ with the stronger condition $\langle\mu, \widetilde{\alpha}\rangle \leqslant 0$ (only in the cases with $i \neq 0$ ), the resulting domain for $\mu$ is bounded by a subset of the root hyperplanes for $W$, and thus is a union of (closed) Weyl chambers in $V$.

Select one of these Weyl chambers $C$, and choose $\mu \in \theta+\mathbf{Z} \Phi^{\vee}$ sufficiently deep in this chamber so that $\mu+\beta_{1}^{\vee}+\beta_{2}^{\vee}$ is in the interior of $C$ for all $\beta_{1}, \beta_{2} \in \Phi$. Setting $\nu:=\mu+\beta_{1}^{\vee}+\beta_{2}^{\vee}$, it is clear that $x(\mu)$ and $x(\nu)$ are both in $(\widetilde{W} x(\theta))_{\langle i\rangle}$.

We further claim that if $\left\langle\mu, \beta_{1}^{\vee}+\beta_{2}^{\vee}\right\rangle \geqslant 0$ and $\left\langle\beta_{1}^{\vee}, \beta_{2}^{\vee}\right\rangle \geqslant 0$, then $x(\mu) \succcurlyeq x(\nu)$. Indeed, by Lemma 4.8, it suffices to show that $c \widetilde{\alpha} \succcurlyeq \beta_{1}^{\vee}+\beta_{2}^{\vee}$ and $c \geqslant 0$, where

$$
\begin{aligned}
c & =\frac{1}{2}\|\nu\|^{2}-\frac{1}{2}\|\mu\|^{2}=\frac{1}{2}\langle\nu+\mu, \nu-\mu\rangle=\frac{1}{2}\left\langle 2 \mu+\beta_{1}^{\vee}+\beta_{2}^{\vee}, \beta_{1}^{\vee}+\beta_{2}^{\vee}\right\rangle \\
& \geqslant \frac{1}{2}\left\|\beta_{1}^{\vee}+\beta_{2}^{\vee}\right\|^{2} \geqslant \frac{1}{2}\left\|\beta_{1}^{\vee}\right\|^{2}+\frac{1}{2}\left\|\beta_{2}^{\vee}\right\|^{2} .
\end{aligned}
$$

Thus it is clear that $c>0$. Furthermore, since $\widetilde{\alpha}$ is the highest root, we have $\widetilde{\alpha} \succcurlyeq \beta_{j}$, or equivalently $\frac{1}{2}\left\|\beta_{j}^{\vee}\right\|^{2} \widetilde{\alpha} \succ \beta_{j}^{\vee}$. Consequently, $c \widetilde{\alpha} \succcurlyeq \beta_{1}^{\vee}+\beta_{2}^{\vee}$ and the claim follows.

Now let $w$ denote the unique element of $W$ such that $w C$ is the dominant chamber. Given the hypothesis that $W$ has rank $\geqslant 2$, we may choose a pair of distinct simple roots, say $\alpha_{1}$ and $\alpha_{2}$, and set $\beta_{1}:=w^{-1} \alpha_{1}$ and $\beta_{2}:=-w^{-1} \alpha_{2}$. Interchanging $\alpha_{1}$ and $\alpha_{2}$ if necessary (which has the effect of replacing $\left(\beta_{1}, \beta_{2}\right)$ with $\left(-\beta_{2},-\beta_{1}\right)$ ), we may assume that $\left\langle\mu, \beta_{1}^{\vee}+\beta_{2}^{\vee}\right\rangle \geqslant 0$. We also have $\left\langle\beta_{1}^{\vee}, \beta_{2}^{\vee}\right\rangle=-\left\langle\alpha_{1}^{\vee}, \alpha_{2}^{\vee}\right\rangle \geqslant 0$, so the hypotheses of the above claim are satisfied, and hence $x(\mu) \succcurlyeq x(\nu)$.

On the other hand, bearing in mind that $\mu$ and $\nu$ are in the interior of the same Weyl chamber $C$, and $w \mu=\mu^{+}$and $w \nu=\nu^{+}$are dominant, we may use Proposition 4.4 to compare the ranks of $x(\mu)$ and $x(\nu)$ in the Bruhat ordering of $\widetilde{W} x(\theta)$, obtaining

$$
\begin{aligned}
r(x(\nu))-r(x(\mu)) & =\sum_{\beta \in \Phi^{+}}\left\lceil\left\langle\nu^{+}, \beta\right\rangle\right\rceil-\left\lceil\left\langle\mu^{+}, \beta\right\rangle\right\rceil \\
& =\sum_{\beta \in \Phi^{+}}\langle w \nu-w \mu, \beta\rangle=\sum_{\beta \in \Phi^{+}}\left\langle\alpha_{1}^{\vee}-\alpha_{2}^{\vee}, \beta\right\rangle=0,
\end{aligned}
$$

the second equality being a consequence of the fact that $\left\langle\nu^{+}, \beta\right\rangle$ and $\left\langle\mu^{+}, \beta\right\rangle$ (or equivalently $\langle\nu, \beta\rangle$ and $\langle\mu, \beta\rangle)$ differ by integers for all roots $\beta$, and the third equality being a consequence of the well-known fact that $\left\langle\alpha_{j}^{\vee}, \rho\right\rangle=1$ for all simple roots $\alpha_{j}$, where $2 \rho$ is the sum of all positive roots (e.g., see Section VI.1.10 of $[\mathbf{B}]$ ). Thus $x(\mu)$ and $x(\nu)$ must be incomparable in the Bruhat order and $(\widetilde{W} x(\theta))_{I}$ cannot be tight.

D. Minuscule double quotients.

Although the $\widetilde{W}$-orbits in $X$ do not afford any nontrivial tight quotients, Corollary 4.7 shows that the identification $x(\mu) \mapsto \mu$ provides an order-preserving map from the Bruhat orderings of the double quotients $(\widetilde{W} x(\theta))_{I}$ with $I=S$ to the standard ordering of $V$, so it is natural to investigate the possibility that this is an order embedding.

One case in which this occurs involves minuscule co-weights.

The lattice of (integral) co-weights, denoted $\Lambda^{\vee}$, consists of all $\mu \in \operatorname{Span} \Phi$ such that $\langle\mu, \beta\rangle \in \mathbf{Z}$ for all $\beta \in \Phi$. Each reflection in $W$ acts on $\mu \in \Lambda^{\vee}$ by adding integer multiples 
of co-roots, so the $\widetilde{W}$-orbit of $x(\mu)$ consists of all points $x(\nu)$ such that $\nu-\mu \in \mathbf{Z} \Phi^{\vee}$. The Bruhat ordering of this orbit necessarily has a minimum element $x(\theta)$; namely, the unique dominant member. Equivalently, $\theta$ may be identified as the unique member of $\mu+\mathbf{Z} \Phi^{\vee}$ in the fundamental alcove $A_{0}$. These minimum co-weights $\theta$ are precisely the co-weights that are minuscule and dominant; i.e., $\langle\theta, \beta\rangle \in\{0,1\}$ for all $\beta \in \Phi^{+}$ (cf. Example 2.2).

THEOREM 4.10. If $\theta$ is a dominant minuscule co-weight (i.e., $\theta \in A_{0} \cap \Lambda^{\vee}$ ) and $\widetilde{W}_{J}$ is the stabilizer of $x(\theta)$, then $\left({ }^{S} \widetilde{W}^{J},<_{\mathrm{B}}\right)$ is isomorphic to the standard ordering of the dominant part of $\theta+\mathbf{Z} \Phi^{\vee}$; i.e., for all dominant $\mu, \nu \in \theta+\mathbf{Z} \Phi^{\vee}$, we have

$$
x(\mu)<_{\mathrm{B}} x(\nu) \Leftrightarrow \mu \prec \nu .
$$

Furthermore, the order dimension of $\left({ }^{S} \widetilde{W}^{J},<_{\mathrm{B}}\right)$ is $|S|$.

The following result is implicit in the work of Moody and Patera [MP]; for a stronger result, see also Theorem 2.6 of $[\mathbf{S 2}]$. We include here a short proof due to R. Steinberg.

LEMMA 4.11. If $\mu, \nu \in \theta+\mathbf{Z} \Phi^{\vee}$ are $\Phi$-dominant and $\mu \prec \nu$, then there is a root $\beta \in \Phi^{+}$such that $\mu+\beta^{\vee}$ is $\Phi$-dominant and $\mu+\beta^{\vee} \preccurlyeq \nu$.

Proof. Given that $\nu-\mu \in \mathbf{Z} \Phi^{\vee}$ and $\mu \prec \nu$, there exist positive roots $\beta_{1}, \ldots, \beta_{l}$ such that $\nu-\mu=\beta_{1}^{\vee}+\cdots+\beta_{l}^{\vee}$. Among all such representations, choose one that maximizes the height of $\beta_{1}^{\vee}$. We claim that $\mu+\beta_{1}^{\vee}$ is $\Phi$-dominant (and thus it suffices to take $\beta=\beta_{1}$ ). If not, then $\left\langle\mu+\beta_{1}^{\vee}, \alpha_{i}\right\rangle<0$ for some $i$, and hence $\left\langle\beta_{1}^{\vee}, \alpha_{i}\right\rangle<0$, since $\mu$ is $\Phi$-dominant. On the other hand, $\nu$ is also $\Phi$-dominant, so after renumbering $\beta_{2}, \ldots, \beta_{l}$ if necessary, it must be the case that $\left\langle\beta_{2}^{\vee}, \alpha_{i}\right\rangle>0$. Now $\left\langle\beta_{1}^{\vee}, \alpha_{i}\right\rangle<0$ implies that $\beta_{1}^{\vee}+\alpha_{i}^{\vee}$ is a co-root and $\left\langle\beta_{2}^{\vee}, \alpha_{i}\right\rangle>0$ implies that $\beta_{2}^{\vee}-\alpha_{i}^{\vee}$ is a (positive) co-root or $\beta_{2}=\alpha_{i}$ (e.g., see Section VI.1.3 of $[\mathbf{B}]$ ). Thus we may replace $\beta_{1}^{\vee}$ with $\beta_{1}^{\vee}+\alpha_{i}^{\vee}$ and $\beta_{2}^{\vee}$ with $\beta_{2}^{\vee}-\alpha_{i}^{\vee}$ (or delete $\beta_{2}^{\vee}$, if $\beta_{2}=\alpha_{i}$ ), contradicting the choice of $\beta_{1}$.

Proof of Theorem 4.10. Assume $\mu, \nu \in \theta+\mathbf{Z} \Phi^{\vee}$ are $\Phi$-dominant. By Corollary 4.7, we know that if $x(\mu)<_{\mathrm{B}} x(\nu)$ is a covering relation, then $\mu \prec \nu$. For the converse, suppose we have $\mu \prec \nu$. By Lemma 4.11, there is a positive $\operatorname{root} \beta$ such that $\mu+\beta^{\vee}$ is $\Phi$-dominant and $\mu+\beta^{\vee} \preccurlyeq \nu$. Proceeding by induction on the length of the longest $\prec$-chain from $\mu$ to $\nu$, it follows that $x\left(\mu+\beta^{\vee}\right) \leqslant_{\mathrm{B}} x(\nu)$, and hence $x(\mu)<_{\mathrm{B}} x\left(\mu+\beta^{\vee}\right) \leqslant_{\mathrm{B}} x(\nu)$ by Lemma 4.5.

The above isomorphism between the Bruhat and standard orders proves that the order dimension of $\left({ }^{S} \widetilde{W}^{J},<_{\mathrm{B}}\right)$ is at most $n=|S|$. To prove that this bound is sharp, choose $\mu \in \theta+\mathbf{Z} \Phi^{\vee}$ sufficiently deep in the dominant chamber so that for every sum $\gamma$ of distinct simple co-roots, $\mu+\gamma$ is dominant. Setting $\nu=\mu+\alpha_{1}^{\vee}+\cdots+\alpha_{n}^{\vee}$, it follows that $\mu+\alpha_{i}^{\vee}$ and $\nu-\alpha_{i}^{\vee}$ are dominant for all $i$. Furthermore, we have

$$
\mu+\alpha_{i}^{\vee} \prec \nu-\alpha_{j}^{\vee} \Leftrightarrow i \neq j
$$

It follows that in every coordinate embedding for this poset of relations, the (nonempty) sets of coordinates where $\mu+\alpha_{i}^{\vee}$ exceeds those of $\nu-\alpha_{i}^{\vee}$ must be disjoint for distinct choices of $i$, and hence there must be at least $n$ such coordinates. 
By Theorem 1.3 of $[\mathbf{S 2}]$, one knows that for each minuscule co-weight $\theta$, the standard ordering of the dominant part of $\theta+\mathbf{Z} \Phi^{\vee}$ is a lattice in the order-theoretic sense (i.e., every pair of elements has a least upper bound and greatest lower bound). Hence,

Corollary 4.12. If $\widetilde{W}_{J}$ is the stabilizer of $x(\theta)$ for some dominant minuscule coweight $\theta$, then $\left({ }^{S} \widetilde{W}^{J},<_{\mathrm{B}}\right)$ is a lattice.

REMARK 4.13. The Bruhat ordering of a (one-sided) parabolic quotient is rarely a lattice. According to the classification (Theorem 7.1 of $[\mathbf{S 1}]$ ), most of the examples involve minuscule orbits of finite Weyl groups as in Example 2.2. The above result suggests that it would be interesting to classify all double quotients whose Bruhat ordering is a lattice.

REMARK 4.14. The dominant minuscule co-weights have many characterizations.

(a) For example, they are necessarily vertices of $A_{0}$, and in the irreducible case may be identified as the vertices $\theta_{i}$ such that $c_{i}=1$ in the expansion

$$
\delta=c_{0} \alpha_{0}+\cdots+c_{n} \alpha_{n}=c_{0}(\delta-\widetilde{\alpha})+c_{1} \alpha_{1}+\cdots+c_{n} \alpha_{n} .
$$

Of course, $\theta_{0}=0$ is minuscule and $c_{0}=1$.

(b) For another characterization, note that for $\theta \in V$, the $\widetilde{W}$-stabilizer of $x(\theta)$ is necessarily generated by reflections. There is one such reflection corresponding to each root of the form $k \delta-\beta$, where $\langle\theta, \beta\rangle=k \in \mathbf{Z}$. It follows that the stabilizer of $x(\theta)$ is isomorphic to the finite reflection group associated to the root system $\Psi=\{\beta \in \Phi$ : $\langle\theta, \beta\rangle \in \mathbf{Z}\}$. In particular, $\theta$ is an integral co-weight if and only if $x(\theta)$ has a stabilizer isomorphic to $W$, and the parabolic subgroups $\widetilde{W}_{J}$ that occur as the stabilizers of (the $X$-images of) minuscule co-weights in $A_{0}$ are precisely those for which $\widetilde{W}_{J} \cong W$.

(c) For a third characterization, note that if $\theta \in \Lambda^{\vee}$ and $\beta \in \Phi$, then $\langle\beta, \mu\rangle \in \mathbf{Z}$ if and only if $\langle\beta, \mu+\theta\rangle \in \mathbf{Z}$, so the translation $t_{\theta}$ permutes the root hyperplanes for $\widetilde{W}$, and hence also permutes the alcoves (i.e., the connected regions in $X$ complementary to the root hyperplanes). However, $\widetilde{W}$ is simply transitive on alcoves, so there is some $w \in \widetilde{W}$ such that as an affine transformation, $w t_{\theta}$ fixes $A_{0}$ setwise and conformally permutes its faces. Since the angles between facets of $A_{0}$ encode the defining relations of $\widetilde{W}$, it follows that $w t_{\theta}$ induces a diagram automorphism $\sigma$ of $\widetilde{W}$. Now if $\theta$ is also a vertex of $A_{0}$ (i.e., dominant minuscule), then by simple transitivity, the permutation of vertices induced by $w t_{\theta}$ must send 0 to $\theta$, and hence $\sigma$ provides an isomorphism between $W=\widetilde{W}_{S}$ and the subgroup $\widetilde{W}_{J}$ that fixes $x(\theta)$. Thus the dominant minuscule co-weights are in bijection with subsets of $\widetilde{S}$ in the orbit of $S$ under the group of diagram automorphisms of $\widetilde{W}$.

The diagram automorphisms of any Coxeter group induce automorphisms of the Bruhat order, so Theorem 4.10 and the preceding remark yield

Corollary 4.15. If $W \cong \widetilde{W}_{I} \cong \widetilde{W}_{J}$ (i.e., $\widetilde{W}_{I}$ and $\widetilde{W}_{J}$ are the $\widetilde{W}$-stabilizers of dominant minuscule co-weights), then the order dimension of $\left({ }^{I} \widetilde{W}^{J},<_{\mathrm{B}}\right)$ is $|S|$. 
In the case $W=S_{n}$, the Coxeter diagram of $\widetilde{W}$ has a vertex-transitive automorphism group. In particular, every maximal parabolic subgroup of $\widetilde{S}_{n}$ is isomorphic to $S_{n}$. Bearing in mind (1.2), we conclude

Corollary 4.16. We have $\operatorname{dim}\left(\widetilde{S}_{n},<_{\mathrm{B}}\right) \leqslant n^{2}(n-1)$.

Later we will improve this bound to $n^{3} / 2+O\left(n^{2}\right)$; a sharper bound of $n^{3} / 4+O\left(n^{2}\right)$ has been obtained by Reading and Waugh $[\mathbf{R W}]$.

\section{Affine embeddings of affine Weyl group quotients.}

Having established that the orbits generated by the reflection representations of affine Weyl groups do not afford any nontrivial tight quotients (Theorem 4.9), we now focus on the prospect (exemplified by Theorem 4.10) of obtaining analogues of tightness derived from the representation of $\widetilde{W}$ as a group of affine transformations of $V$. This leads to the notion of tight affine embeddings of (subposets of) the Bruhat order, and culminates in the classification of all double quotients $\left({ }^{I} \widetilde{W}^{J},<_{\mathrm{B}}\right)$ with such embeddings.

For simplicity, we will assume throughout that $\widetilde{W}$ is irreducible.

\section{A. Tight affine embeddings.}

In order to avoid confusion with the linear representation of $\widetilde{W}$ on $\widetilde{V}$, we will write $w . \mu$ for the affine action of $w \in \widetilde{W}$ on $\mu \in V$. Thus,

$$
t_{\nu} \cdot \mu=\nu+\mu \quad\left(\nu \in \mathbf{Z} \Phi^{\vee}\right), \quad s_{\beta} \cdot \mu=\mu-\langle\mu, \beta\rangle \beta^{\vee} \quad(\beta \in \Phi) .
$$

Via the identification between $V$ and the cross section $X$, each orbit $\widetilde{W} . \theta$ carries a Bruhat ordering isomorphic to $\left(\widetilde{W} x(\theta),<_{\mathrm{B}}\right)$, and for each $I \subset \widetilde{S}$, we let $(\widetilde{W} \cdot \theta)_{I}$ denote the pre-image in $V$ of the double quotient $(\widetilde{W} x(\theta))_{I}$.

We define a tight (affine) embedding of the Bruhat ordering of a subset $Y$ of $\widetilde{W} . \theta$ to be a linear map $f: V \rightarrow V$ such that for all $\mu, \nu \in Y$, we have

$$
\mu \leqslant \mathrm{B} \nu \Leftrightarrow f(\mu) \preccurlyeq f(\nu) .
$$

Our goal is to identify the double quotients $\left((\widetilde{W} \cdot \theta)_{I},<_{\mathrm{B}}\right)$ that have tight embeddings.

We say that $I \subset \widetilde{S}$ is of minuscule type if $\widetilde{W}_{I}$ is the $\widetilde{W}$-stabilizer of a dominant minuscule co-weight, or equivalently, $\widetilde{W}_{I} \cong W$ (see Remark 4.14(b)).

Note that $S$ is always of minuscule type.

TheOREM 5.1. If $\left((\widetilde{W} \cdot \theta)_{I},<_{\mathrm{B}}\right)$ has a tight embedding and $I \neq \widetilde{S}$, then $I$ is of minuscule type. Furthermore, in the case $I=S$, if there is any tight embedding, then the identity map suffices; i.e.,

$$
\mu \leqslant \mathrm{B} \nu \Leftrightarrow \mu \preccurlyeq \nu \quad\left(\mu, \nu \in(\widetilde{W} \cdot \theta)_{S}\right) .
$$

Proof. Suppose that $f: V \rightarrow V$ provides a tight embedding of $\left((\widetilde{W} \cdot \theta)_{I},<_{\mathrm{B}}\right)$. Note that $(\widetilde{W} \cdot \theta)_{I}$ includes every $\mu \in \theta+\mathbf{Z} \Phi^{\vee}$ in the cone $C$ defined by the inequalities

$$
\left.\left\langle\mu, \alpha_{j}\right\rangle \geqslant 0 \quad \text { (if } s_{j} \in I-\left\{s_{0}\right\}\right), \quad\langle\mu, \widetilde{\alpha}\rangle \leqslant 1 \quad\left(\text { if } s_{0} \in I\right) .
$$


In case $s_{0} \in I$, let $C_{0}$ denote the sub-cone of $C$ satisfying $\langle\mu, \widetilde{\alpha}\rangle \leqslant 0$; otherwise, set $C_{0}:=C$. Since $I \neq \widetilde{S}$ and $\widetilde{\alpha}$ is not in the span of any proper subset of $\left\{\alpha_{1}, \ldots, \alpha_{n}\right\}$, it follows that $C_{0}$ has a non-empty interior. Furthermore, since $C_{0}$ is bounded by root hyperplanes, it must be a union of one or more (closed) Weyl chambers.

If it happens that $C_{0}$ includes at least two Weyl chambers, then there is at least one root hyperplane that separates two of them. That is, there is a root $\beta \in \Phi^{+}$and a pair of Weyl chambers $C_{ \pm} \subset C$ such that $\langle\mu, \beta\rangle \geqslant 0$ for $\mu \in C_{+}$and $\langle\mu, \beta\rangle \leqslant 0$ for $\mu \in C_{-}$. If we choose $\mu \in \theta+\mathbf{Z} \Phi^{\vee}$ sufficiently deep in the chamber $C_{+}$so that $\mu+\beta^{\vee} \in C_{+}$, and similarly choose $\nu \in C_{-}$so that $\nu-\beta^{\vee} \in C_{-}$, then each of $\mu, \nu, \mu+\beta^{\vee}, \nu-\beta^{\vee}$ will be points of $(\widetilde{W} \cdot \theta)_{I}$, and it follows via Lemma 4.5 that $\mu<_{\mathrm{B}} \mu+\beta^{\vee}$ and $\nu<_{\mathrm{B}} \nu-\beta^{\vee}$. Given that we have a tight embedding, we must therefore have $f(\mu) \prec f(\mu)+f\left(\beta^{\vee}\right)$ and $f(\nu) \prec f(\nu)-f\left(\beta^{\vee}\right)$; i.e., $f\left(\beta^{\vee}\right) \succ 0$ and $f\left(\beta^{\vee}\right) \prec 0$, a contradiction.

Thus the cone $C_{0}$ must consist of a single Weyl chamber, so there must exist $w \in W$ that transforms $C_{0}$ to the dominant chamber. In particular, the reflections through the walls of $C_{0}$ are conjugate to $S$, so $\widetilde{W}_{I} \cong W$ and $I$ must be of minuscule type.

To prove the second assertion, assume now that we are in the (minuscule) case $I=S$. Here, the cone $C$ is the dominant chamber, and by choosing $\mu \in \theta+\mathbf{Z} \Phi^{\vee}$ sufficiently deep in this chamber, we will have $\mu+\alpha_{j}^{\vee}$ dominant and $\mu<_{\mathrm{B}} \mu+\alpha_{j}^{\vee}$ for all $j$, and hence $f\left(\alpha_{j}^{\vee}\right) \succ 0$ for all $j$. It follows that $\mu \preccurlyeq \nu \Rightarrow f(\mu) \preccurlyeq f(\nu)$ for all $\mu, \nu \in V$, and we know conversely that $\mu \leqslant_{\mathrm{B}} \nu \Rightarrow \mu \preccurlyeq \nu$ for all $\mu, \nu \in(\widetilde{W} \cdot \theta)_{S}$ (Corollary 4.7), so there is no loss of generality in choosing $f$ to be the identity map in the case $I=S$.

Thus by Remark 4.14(c), the classification of all double quotients ${ }^{I} \widetilde{W}^{J}$ whose Bruhat ordering admits a tight affine embedding in $V$ may be reduced via automorphisms to the identification of all points $\theta \in A_{0}$ such that (5.1) holds. In such cases, we say that the Bruhat ordering of $(\widetilde{W} \cdot \theta)_{S}$ is tight.

\section{B. Orbit stratification.}

Recall that the tightness of the Bruhat ordering of a $W$-orbit is controlled by the stratification of orbits of parabolic subgroups (Corollary 3.10). It turns out that there is a similar phenomenon that controls the tightness of $\left((\widetilde{W} \cdot \theta)_{S},<_{\mathrm{B}}\right)$; however, it involves orbits of parabolic subgroups in the action of $W$ on $V / \mathbf{Z} \Phi^{\vee}$ (a torus, if $V=\operatorname{Span} \Phi$ ).

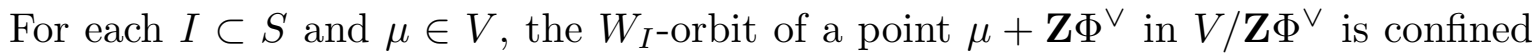
to $\mu+\operatorname{Span} \Phi_{I}+\mathbf{Z} \Phi^{\vee}$. Thus $\mu+\mathbf{Z} \Phi^{\vee}$ and $\nu+\mathbf{Z} \Phi^{\vee}$ cannot be in the same $W_{I^{-}}$orbit in $V / \mathbf{Z} \Phi^{\vee}$ unless $\mu-\nu \in \operatorname{Span} \Phi_{I}+\mathbf{Z} \Phi^{\vee}$. If this criterion is sufficient to distinguish the $W_{I^{-}}$orbits in the $W$-orbit of $\theta+\mathbf{Z} \Phi^{\vee}$ (i.e., for all $\mu, \nu \in W \theta$, we have $\mu+\mathbf{Z} \Phi^{\vee}$ and $\nu+\mathbf{Z} \Phi^{\vee}$ in the same $W_{I^{-}}$orbit if and only if $\left.\mu-\nu \in \operatorname{Span} \Phi_{I}+\mathbf{Z} \Phi^{\vee}\right)$, then we say that

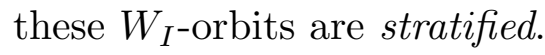

Proposition 5.2. If $\theta \in A_{0}$ has stabilizer $\widetilde{W}_{J}$ and $\left((\widetilde{W} . \theta)_{S},<_{\mathrm{B}}\right)$ is tight, then

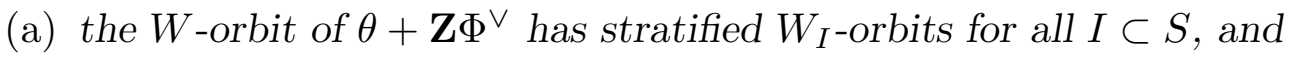

(b) for all $\theta^{\prime} \in A_{0}$ fixed by $\widetilde{W}_{J}$, the $W$-orbit of $\theta^{\prime}+\mathbf{Z} \Phi^{\vee}$ has stratified $W_{\langle i\rangle}$-orbits. 
Proof. We seek to show that the $W$-orbit of $\theta^{\prime}+\mathbf{Z} \Phi^{\vee}$ has stratified $W_{I^{-}}$-orbits if either $\theta=\theta^{\prime}$ or $I=\langle i\rangle$ for some $i$. Now since the $W$-orbit of $\theta^{\prime}+\mathbf{Z} \Phi^{\vee}$ may be identified with the $\widetilde{W}$-orbit of $\theta^{\prime}$ modulo translation, it therefore suffices to prove that for all $x, y \in \widetilde{W}$ such that $x . \theta^{\prime}-y . \theta^{\prime} \in \operatorname{Span} \Phi_{I}$, we have $x . \theta^{\prime}+\mathbf{Z} \Phi^{\vee}$ and $y . \theta^{\prime}+\mathbf{Z} \Phi^{\vee}$ in the same $W_{I}$-orbit.

In the case $I=\langle i\rangle$, we may interchange $x$ and $y$ if necessary so that the coefficient of $\alpha_{i}$ in $y . \theta-x . \theta$ is nonnegative; note that the analogous coefficient in $y \cdot \theta^{\prime}-x \cdot \theta^{\prime}$ is zero. In the case $\theta=\theta^{\prime}$, the coefficient of $\alpha_{i}$ in $y . \theta-x . \theta$ is zero for all $s_{i} \in S-I$. Thus in both cases, we may replace $y$ with a translate $t_{\mu} y$ for a suitably chosen $\mu \in \mathbf{Z} \Phi_{I}^{\vee}$ so that

$$
x . \theta \preccurlyeq y . \theta \quad \text { and } \quad x . \theta^{\prime}-y . \theta^{\prime} \in \operatorname{Span} \Phi_{I} .
$$

By choosing $\nu \in \mathbf{Z} \Phi^{\vee}$ sufficiently deep in the dominant chamber and replacing $x$ and $y$ with $t_{\nu} x$ and $t_{\nu} y$, we may further assume that $x . \theta, x . \theta^{\prime}, y . \theta$ and $y . \theta^{\prime}$ are all dominant.

Given that $\left((\widetilde{W} . \theta)_{S},<_{\mathrm{B}}\right)$ is tight, it follows that $x . \theta \leqslant_{\mathrm{B}} y . \theta$. Furthermore, since the stabilizer of $\theta^{\prime}$ includes the stabilizer of $\theta$, the map $w \cdot \theta \mapsto w \cdot \theta^{\prime}$ is well-defined and order-preserving (Proposition 1.1), so we have $x \cdot \theta^{\prime} \leqslant_{\mathrm{B}} y \cdot \theta^{\prime}$. However, Corollary 4.7 shows that every Bruhat chain from $x . \theta^{\prime}$ to $y . \theta^{\prime}$ involves adding a sequence of positive multiples of positive roots. Since $y . \theta^{\prime}-x \cdot \theta^{\prime} \in \operatorname{Span} \Phi_{I}$, these roots must be in $\Phi_{I}$, and hence there is a sequence of affine reflections $s_{k \delta+\beta}\left(k \in \mathbf{Z}, \beta \in \Phi_{I}\right)$ that transform $x . \theta^{\prime}$ to $y \cdot \theta^{\prime}$. In other words, $x \cdot \theta^{\prime}+\mathbf{Z} \Phi^{\vee}$ and $y \cdot \theta^{\prime}+\mathbf{Z} \Phi^{\vee}$ are in the same $W_{I^{-}}$orbit.

REMARK 5.3. (a) For $\theta \in A_{0}$, the stabilizer $W_{\theta}$ of $\theta+\mathbf{Z} \Phi^{\vee}$ in $W$ is the image of a parabolic subgroup of $\widetilde{W}$ (the $\widetilde{W}$-stabilizer of $\theta$ ) under the natural map $\widetilde{W} \rightarrow W$, and thus is generated by reflections. However, $W_{\theta}$ need not be a parabolic subgroup of $W$.

(b) One way to test whether the $W$-orbit of $\theta+\mathbf{Z} \Phi^{\vee}$ has stratified $W_{I^{-} \text {-orbits for all }}$ $I \subset S$ is to first select representatives $\nu_{1}, \ldots, \nu_{l} \in V$ for the points in the orbit (where $l$ denotes the index of $W_{\theta}$ in $W$ ); i.e.,

$$
W \theta+\mathbf{Z} \Phi^{\vee}=\left(\nu_{1}+\mathbf{Z} \Phi^{\vee}\right) \dot{U} \cdots \dot{U}\left(\nu_{l}+\mathbf{Z} \Phi^{\vee}\right) .
$$

By normalizing each representative $\nu_{i}=\theta-\sum a_{j} \alpha_{j}^{\vee}$ so that $0 \leqslant a_{j}<1$ for all $j$, we claim that the $W_{I^{-}}$-rbits are stratified for all $I$ if and only if for all $i \neq j, \nu_{i}+\mathbf{Z} \Phi^{\vee}$ and $\nu_{j}+\mathbf{Z} \Phi^{\vee}$ are in the same $W_{J}$-orbit, where $J$ is the smallest subset of $S$ such that $\nu_{i}-\nu_{j} \in \operatorname{Span} \Phi_{J}$. Certainly this condition is necessary; for the sufficiency, suppose we have $\nu_{i}-\nu_{j} \in \operatorname{Span} \Phi_{I}+\mathbf{Z} \Phi^{\vee}$ for some $I \subset S$. For each $s_{k} \notin I$, the coefficient of $\alpha_{k}^{\vee}$ in $\nu_{i}-\nu_{j}$ must be an integer; however, given our normalizing convention, this coefficient is strictly between -1 and 1 , and therefore vanishes; i.e., $s_{k} \notin J$. We thus have $J \subseteq I$, so the fact that $\nu_{i}+\mathbf{Z} \Phi^{\vee}$ and $\nu_{j}+\mathbf{Z} \Phi^{\vee}$ are in the same $W_{J}$-orbit also places them in the same $W_{I^{-} \text {-orbit. }}$

Now suppose that $\theta \in A_{0}$ is a half-integral co-weight (i.e., $\langle\theta, \beta\rangle \in \mathbf{Z} / 2$ for all $\beta \in \Phi$ ) and satisfies the constraint

$$
\beta \in \Phi \text { is long } \Rightarrow\langle\theta, \beta\rangle \in \mathbf{Z} .
$$

If all roots have the same length, regard them as short; in that case, (5.2) is vacuous. We remark that in the case $W=G_{2}$, the only (nonzero) half-integral co-weight in $A_{0}$ is $\theta_{2}=(1 / 2) \widetilde{\alpha}^{\vee}$. However, $\left\langle\theta_{2}, \alpha_{2}\right\rangle=1 / 2 \notin \mathbf{Z}$ and $\alpha_{2}$ is long. 
LEMmA 5.4. If $\theta \in A_{0}$ is a half-integral co-weight such that (5.2) holds, then for all $\mu \in \widetilde{W} . \theta$, we have $\mu=\theta+\sum b_{i} \alpha_{i}^{\vee}$, where $b_{i} \in \mathbf{Z} / 2$ for all $i$ and $b_{i} \in \mathbf{Z}$ if $\alpha_{i}$ is long.

Proof. It suffices to show that

$$
L:=\left\{\theta+\sum b_{i} \alpha_{i}^{\vee}: b_{i} \in \mathbf{Z} / 2 ; \alpha_{i} \text { long } \Rightarrow b_{i} \in \mathbf{Z}\right\}
$$

is $\widetilde{W}$-stable. Certainly it is stable under translation by $\mathbf{Z} \Phi^{\vee}$, so we need only to check that it is stable with respect to each simple reflection $s_{j} \in W$. For this, let us choose $\mu=\theta+\sum b_{i} \alpha_{i}^{\vee} \in L$ and note that $\mu$ is clearly a half-integral co-weight, so if $\alpha_{j}$ is short, the coefficient of $\alpha_{j}^{\vee}$ in $s_{j} \mu=\mu-\left\langle\mu, \alpha_{j}\right\rangle \alpha_{j}^{\vee}$ remains half-integral and there is nothing further to check. On the other hand, if $\alpha_{j}$ is long, then we have $\left\langle\alpha_{i}^{\vee}, \alpha_{j}\right\rangle \in 2 \mathbf{Z}$ for all short $\alpha_{i}$ (recall from the above remark that $W \neq G_{2}$ except in the trivial case $\theta=0$ ), and hence $b_{i}\left\langle\alpha_{i}^{\vee}, \alpha_{j}\right\rangle \in \mathbf{Z}$ for all $i$. Thus $\left\langle\mu, \alpha_{j}\right\rangle \in \mathbf{Z}$ and the coefficient of $\alpha_{j}^{\vee}$ in $s_{j} \mu$ remains integer-valued.

In case $\theta \in A_{0}$ is an integral (hence minuscule) co-weight, (5.2) is vacuous and the $W$-orbit of $\theta+\mathbf{Z} \Phi^{\vee}$ is trivial (so orbit stratification is immediate). Thus the following result may be viewed as a generalization of Theorem 4.10.

THEOREM 5.5. If $\theta \in A_{0}$ is a half-integral co-weight satisfying (5.2), and the $W$-orbit of $\theta+\mathbf{Z} \Phi^{\vee}$ has stratified $W_{I}$-orbits for all $I \subset S$, then $\left((\widetilde{W} . \theta)_{S},<_{\mathrm{B}}\right)$ is tight.

Proof. Given a dominant pair $\mu, \nu \in \widetilde{W} \cdot \theta$ such that $\mu \prec \nu$, we argue by induction on the length of the longest $\prec$-chain from $\mu$ to $\nu$ that $\mu<_{\mathrm{B}} \nu$.

Case 1. There exists $\beta \in \Phi^{+}$such that $\langle\mu, \beta\rangle \in \mathbf{Z}+1 / 2$ and $\mu+(1 / 2) \beta^{\vee} \preccurlyeq \nu$.

In this case, $\beta$ is necessarily short, by (5.2). We claim that if $\beta$ is chosen to maximize height, then $\mu+(1 / 2) \beta^{\vee}$ is necessarily dominant. Given this claim, we are done, since $\mu<_{\mathrm{B}} \mu+(1 / 2) \beta^{\vee} \preccurlyeq \nu$ by (4.1a), so we may replace $\mu$ with $\mu+(1 / 2) \beta^{\vee}$ and continue the induction. Thus toward a contradiction, we suppose $\left\langle\mu+(1 / 2) \beta^{\vee}, \alpha_{j}\right\rangle<0$ for some $j$, whence $\left\langle\beta^{\vee}, \alpha_{j}\right\rangle<0$ since $\mu$ is dominant.

If $\alpha_{j}$ is short, then we have $\left\langle\beta^{\vee}, \alpha_{j}\right\rangle=\left\langle\alpha_{j}^{\vee}, \beta\right\rangle=-1$ and $s_{j} \beta^{\vee}=\beta^{\vee}+\alpha_{j}^{\vee}=\left(\beta+\alpha_{j}\right)^{\vee}$. Moreover, $\left\langle\mu, \alpha_{j}\right\rangle \in \mathbf{Z} / 2$ and $\left\langle\mu+(1 / 2) \beta^{\vee}, \alpha_{j}\right\rangle=\left\langle\mu, \alpha_{j}\right\rangle-1 / 2<0$, so it must be the case that $\left\langle\mu, \alpha_{j}\right\rangle=0$ and $\left\langle\mu, \beta+\alpha_{j}\right\rangle \in \mathbf{Z}+1 / 2$. This contradicts our choice of $\beta$ unless $\mu+(1 / 2)\left(\beta^{\vee}+\alpha_{j}^{\vee}\right) \npreceq \nu$. However by Lemma 5.4, this forces the coefficient of $\alpha_{j}^{\vee}$ in $\nu-\mu-(1 / 2) \beta^{\vee}$ to vanish. Since $\nu-\mu-(1 / 2) \beta^{\vee} \succcurlyeq 0$ and $\left\langle\alpha_{i}^{\vee}, \alpha_{j}\right\rangle \leqslant 0$ for all $i \neq j$, it follows that $\left\langle\nu-\mu-(1 / 2) \beta^{\vee}, \alpha_{j}\right\rangle \leqslant 0$ and hence $\left\langle\nu, \alpha_{j}\right\rangle \leqslant\left\langle\mu+(1 / 2) \beta^{\vee}, \alpha_{j}\right\rangle<0$, contradicting the fact that $\nu$ is dominant.

If $\alpha_{j}$ is long, then as we have noted previously, $W \neq G_{2}$ (unless $\theta=0, \widetilde{W} . \theta=\mathbf{Z} \Phi^{\vee}$, and Case 1 does not apply), so $\left\langle\beta^{\vee}, \alpha_{j}\right\rangle<0$ implies $\left\langle\beta^{\vee}, \alpha_{j}\right\rangle=-2,\left\langle\alpha_{j}^{\vee}, \beta\right\rangle=-1$, and $s_{j} \beta^{\vee}=\beta^{\vee}+2 \alpha_{j}^{\vee}=\left(\beta+\alpha_{j}\right)^{\vee}$. Hence $\left\langle\mu+(1 / 2) \beta^{\vee}, \alpha_{j}\right\rangle=\left\langle\mu, \alpha_{j}\right\rangle-1<0$, and we know $\left\langle\mu, \alpha_{j}\right\rangle \in \mathbf{Z}$ by (5.2), whence $\left\langle\mu, \alpha_{j}\right\rangle=0$ and $\left\langle\mu, \beta+\alpha_{j}\right\rangle \in \mathbf{Z}+1 / 2$. This contradicts our choice of $\beta$ unless $\mu+(1 / 2) \beta^{\vee}+\alpha_{j}^{\vee} \npreceq \nu$. However we know that the coefficient of $\alpha_{j}^{\vee}$ in $\nu-\mu-(1 / 2) \beta^{\vee}$ must be integral (Lemma 5.4), so this is possible only if the coefficient of $\alpha_{j}^{\vee}$ in $\nu-\mu-(1 / 2) \beta^{\vee}$ is zero, and hence $\left\langle\nu, \alpha_{j}\right\rangle \leqslant\left\langle\mu+(1 / 2) \beta^{\vee}, \alpha_{j}\right\rangle<0$, again contradicting the fact that $\nu$ is dominant. 
Case 2. For every $\beta \in \Phi^{+}$, either $\langle\mu, \beta\rangle \in \mathbf{Z}$ or $\mu+(1 / 2) \beta^{\vee} \npreceq \nu$.

Let us define $I=\left\{s_{i} \in S:\left\langle\mu, \alpha_{i}\right\rangle \in \mathbf{Z}\right\}$. For each $i$ such that $s_{i} \in S-I$, we have $\left\langle\mu, \alpha_{i}\right\rangle \in \mathbf{Z}+1 / 2$ and hence $\mu+(1 / 2) \alpha_{i}^{\vee} \npreceq \nu$. Bearing in mind Lemma 5.4, this forces $\nu-\mu \in \operatorname{Span} \Phi_{I}$. Given the hypothesis of orbit stratification, it follows that $\nu+\mathbf{Z} \Phi^{\vee}$ and $\mu+\mathbf{Z} \Phi^{\vee}$ must belong to the same $W_{I^{-}}$-orbit. However, $\mu$ is an integral

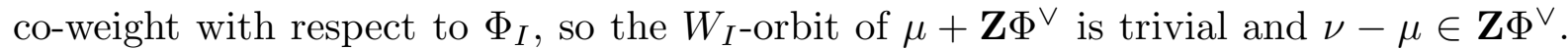
By Lemma 4.11 (note that it does not require $\theta$ to be an integral co-weight), it follows that there is a root $\beta \in \Phi$ such that $\mu+\beta^{\vee}$ is dominant and $\mu \prec \mu+\beta^{\vee} \preccurlyeq \nu$. Thus we have $\mu<_{\mathrm{B}} \mu+\beta^{\vee} \preccurlyeq \nu$ (Lemma 4.5) and the induction continues.

For the numbering of the vertices of $A_{0}$ in what follows, refer to Figure 4.

Corollary 5.6. The Bruhat orderings of the double quotients $\left(\widetilde{B}_{n} \cdot \theta_{1}\right)_{S},\left(\widetilde{C}_{n} \cdot \theta_{2}\right)_{S}$, $\left(\widetilde{C}_{n} \cdot \theta_{n}\right)_{S}$ and $\left(\widetilde{F}_{4} \cdot \theta_{1}\right)_{S}$ are tight.

Proof. For each relevant vertex $\theta_{j}$, the coefficient of $\alpha_{j}$ in $\widetilde{\alpha}$ is 2 , so $\left\langle\theta_{j}, \alpha_{i}\right\rangle=(1 / 2) \delta_{i j}$ and it is clear that $\theta_{j}$ is a half-integral co-weight. Note also that $\alpha_{j}$ is short, so $\left\langle\theta_{j}, \beta\right\rangle=$ $0 \in \mathbf{Z}$ for each long simple root $\beta$. Thus to verify (5.2), it suffices to show that if $\beta$ is long and $\langle\theta, \beta\rangle \in \mathbf{Z}$, then $\left\langle\theta, s_{i} \beta\right\rangle \in \mathbf{Z}$. For this, note that $\left\langle\theta_{j}, \beta\right\rangle-\left\langle\theta, s_{i} \beta\right\rangle=\left\langle\theta_{j}, \alpha_{i}\right\rangle\left\langle\beta, \alpha_{i}^{\vee}\right\rangle$ is an integer, since either $\left\langle\theta_{j}, \alpha_{i}\right\rangle=0$ or $\alpha_{i}$ is short and $\left\langle\beta, \alpha_{i}^{\vee}\right\rangle$ is even. Finally, to show that the $W$-orbit of $\theta_{j}+\mathbf{Z} \Phi^{\vee}$ has stratified $W_{I}$-orbits (thereby permitting the application of Theorem 5.5), we use the technique described in Remark 5.3(b).

In the case $W=B_{n}$, the $W$-stabilizer of $\theta_{1}+\mathbf{Z} \Phi^{\vee}$ is isomorphic to $D_{n}$ (as may be seen from the diagram of $\widetilde{B}_{n}$ ), and thus has index two. It follows that $\theta_{1}$ and $s_{1} \theta_{1}=\theta_{1}-(1 / 2) \alpha_{1}^{\vee}$ are the normalized representatives from the two cosets in $\widetilde{W} \cdot \theta_{1}$, and the stratification of $W_{I}$-orbits is an immediate consequence of Remark 5.3(b).

In the standard coordinates for $C_{n}$, we have $\alpha_{1}=2 \varepsilon_{1}, \alpha_{i}=\varepsilon_{i}-\varepsilon_{i-1}(1<i \leqslant n)$, $\theta_{2}=(1 / 2)\left(\varepsilon_{2}+\cdots+\varepsilon_{n}\right)$, and $\theta_{n}=(1 / 2) \varepsilon_{n}$. The lattice $\mathbf{Z} \Phi^{\vee}$ is generated by $\varepsilon_{1}, \ldots, \varepsilon_{n}$, and the normalized representatives for the cosets of $\widetilde{W} \cdot \theta_{n}$ and $\widetilde{W} \cdot \theta_{2}$ are

$$
\begin{array}{ll}
\nu_{i}=\frac{1}{2} \varepsilon_{i}=\theta_{n}-\frac{1}{2}\left(\alpha_{i+1}^{\vee}+\cdots+\alpha_{n}^{\vee}\right) & (1 \leqslant i \leqslant n), \\
\nu_{i}^{\prime}=\frac{1}{2}\left(\varepsilon_{1}+\cdots+\varepsilon_{n}\right)-\frac{1}{2} \varepsilon_{i}=\theta_{2}-\frac{1}{2}\left(\alpha_{2}^{\vee}+\cdots+\alpha_{i}^{\vee}\right) & (1 \leqslant i \leqslant n),
\end{array}
$$

respectively. For all $i \neq j$, we have $\nu_{i}-\nu_{j}=(1 / 2) \beta^{\vee}$ and $s_{\beta}\left(\nu_{i}\right)=\nu_{j}$ where $\beta$ is the root $\varepsilon_{i}-\varepsilon_{j}$ (and similarly for $\nu_{i}^{\prime}$ and $\nu_{j}^{\prime}$ ), so the stratification of $W_{I^{-}}$-orbits in both cases follows via Remark 5.3(b).

In the case $W=F_{4}$, we have $\theta_{1}=\alpha_{1}^{\vee}+(3 / 2) \alpha_{2}^{\vee}+2 \alpha_{3}^{\vee}+\alpha_{4}^{\vee}$. In particular, $\theta_{1}-(1 / 2) \alpha_{2}^{\vee}$ is in the co-root lattice (i.e., the $\widetilde{W}$-orbit of $\theta_{0}=0$ ) and hence not in $\widetilde{W} \cdot \theta_{1}$. Since $\alpha_{1}$ and $\alpha_{2}$ are the only short simple roots, Lemma 5.4 implies that the only other possibilities for normalized representatives from the cosets of $\widetilde{W} . \theta_{1}$ are $\nu_{1}=\theta_{1}, \nu_{2}=\theta_{1}-(1 / 2) \alpha_{1}^{\vee}$, and $\nu_{3}=\theta_{1}-(1 / 2)\left(\alpha_{1}^{\vee}+\alpha_{2}^{\vee}\right)$. On the other hand, the stabilizer of $\theta_{1}+\mathbf{Z} \Phi^{\vee}$ is $B_{4}$, which has index 3 in $F_{4}$, so these three must be the representatives. Furthermore, we have $\nu_{1}-\nu_{2}=(1 / 2) \alpha_{1}^{\vee}, \nu_{2}-\nu_{3}=(1 / 2) \alpha_{2}^{\vee}$ and $\nu_{1}-\nu_{3}=(1 / 2)\left(\alpha_{1}^{\vee}+\alpha_{2}^{\vee}\right)$, and it is easy to check that $s_{1} \nu_{1}=\nu_{2}, s_{2} \nu_{2}=\nu_{3}$, and $s_{2} s_{1} \nu_{1}=\nu_{3}$, so again the stratification of $W_{I}$-orbits follows via Remark 5.3(b). 


\section{The double affine weight arrangement.}

As in the case of Bruhat orderings of $W$-orbits, there is a relationship between the set of tight double quotients $\left((\widetilde{W} \cdot \theta)_{S},<_{\mathrm{B}}\right)$ and the faces of an associated hyperplane arrangement. Recalling that $\omega_{i}$ denotes the $i$-th fundamental weight, we define the double affine weight arrangement $\widetilde{\mathcal{H}}=\widetilde{\mathcal{H}}(\Phi)$ to be the set of all hyperplanes of the form

$$
H(x, y, i, k):=\left\{\mu \in V:\left\langle\mu, x \omega_{i}-y \omega_{i}\right\rangle=k\right\},
$$

where $x, y \in W, k \in \mathbf{Z}, 1 \leqslant i \leqslant n$, and $x \omega_{i}-y \omega_{i} \neq 0$. Note that this includes the hyperplanes in the double weight arrangement $\mathcal{H}$ of Section 3.

LEMmA 5.7. If $\theta \in A_{0}$ has stabilizer $\widetilde{W}_{J}$ and $\left((\widetilde{W} \cdot \theta)_{S},<_{\mathrm{B}}\right)$ is tight, then there is a face of $\widetilde{\mathcal{H}}$ that contains every $\theta^{\prime} \in A_{0}$ with stabilizer $\widetilde{W}_{J}$.

Proof. Toward a contradiction, suppose that there is a hyperplane $H \in \widetilde{\mathcal{H}}$ such that the points $\theta^{\prime} \in A_{0}$ with stabilizer $\widetilde{W}_{J}$ are not confined to one side of $H$. In that case, there must exist such points $\theta^{\prime}$ that lie on $H$; i.e., $\left\langle\theta^{\prime}, x \omega_{i}-y \omega_{i}\right\rangle \in \mathbf{Z}$ for some $x, y \in W$ and some $i$, and hence $x^{-1} \theta^{\prime}-y^{-1} \theta^{\prime} \in \operatorname{Span} \Phi_{\langle i\rangle}+\mathbf{Z} \alpha_{i}^{\vee}$. By Proposition 5.2(b), we know that the $W_{\langle i\rangle}$-orbits in the $W$-orbit of $\theta^{\prime}+\mathbf{Z} \Phi^{\vee}$ are stratified, so it must be the case that $x^{-1} \theta^{\prime}+\mathbf{Z} \Phi^{\vee}$ and $y^{-1} \theta^{\prime}+\mathbf{Z} \Phi^{\vee}$ are in the same $W_{\langle i\rangle}$-orbit.

On the other hand, if $\theta^{\prime \prime} \in A_{0}$ and $\theta^{\prime}$ both have stabilizer $\widetilde{W}_{J}$, then $\theta^{\prime \prime}+\mathbf{Z} \Phi^{\vee}$ and $\theta^{\prime}+\mathbf{Z} \Phi^{\vee}$ also have the same $W$-stabilizer (namely, the image of $W_{J}$ under the natural map $\widetilde{W} \rightarrow W$ ), and thus $x^{-1} \theta^{\prime \prime}+\mathbf{Z} \Phi^{\vee}$ and $y^{-1} \theta^{\prime \prime}+\mathbf{Z} \Phi^{\vee}$ belong to the same $W_{\langle i\rangle}$-orbit. However, we may choose $\theta^{\prime \prime}$ off of (but close to) $H$, in which case the coefficient of $\alpha_{i}^{\vee}$ in $x^{-1} \theta^{\prime \prime}-y^{-1} \theta^{\prime \prime}$ will not be an integer, a contradiction.

LEMma 5.8. If the face of $A_{0}$ with stabilizer $\widetilde{W}_{J}$ is confined to a single face of $\widetilde{\mathcal{H}}$, then every pair $s_{i}, s_{j} \in \widetilde{S}-J$ must be adjacent in the diagram of $\widetilde{W}$, and if the edge between $s_{i}$ and $s_{j}$ is simple (i.e., $\left(s_{i} s_{j}\right)^{3}=1$ ), then $W$ must be of type $A$.

Proof. Let $F_{J}$ denote the face of $A_{0}$ with stabilizer $\widetilde{W}_{J}$. If $i, j \neq 0$, then Lemma 3.4 shows that $F_{J}$ is not confined to a single face of $\mathcal{H}$, and hence also $\widetilde{\mathcal{H}}$, unless the stated conditions on $s_{i}$ and $s_{j}$ hold.

For the cases involving (say) $i=0$, we may choose $\theta \in F_{J}$ arbitrarily close to 0 , and the line segment $\mu(t):=(1-t) \theta+t \theta_{j}(0<t<1)$ will be entirely contained in $F_{J}$. Now in all cases except those involving $A_{n}$ and $C_{n}$, the highest root $\widetilde{\alpha}$ is a fundamental weight, and hence $H=\{\mu:\langle\mu, 2 \widetilde{\alpha}\rangle=1\}$ is one of the hyperplanes of $\widetilde{\mathcal{H}}$. Thus by choosing $t$ near $1 / 2$ and $\theta \in F_{J}$ sufficiently close to 0 , we see that $\mu(t)$ cannot be confined to one side of $H$, a contradiction whether or not $s_{0}$ and $s_{j}$ are adjacent. For the cases involving $C_{n}$, the short dominant root $\bar{\alpha}=\widetilde{\alpha}-\alpha_{n}$ is a fundamental weight (namely, $\omega_{n-1}$ ), and hence $H^{\prime}=\left\{\mu:\left\langle\mu, 2 \widetilde{\alpha}-2 \alpha_{n}\right\rangle=1\right\}$ is also one of the hyperplanes of $\widetilde{\mathcal{H}}$. Again by choosing $t$ near $1 / 2$ and $\theta \in F_{J}$ near 0 , we see that $\mu(t)$, except in the one case where $s_{0}$ and $s_{j}$ are adjacent by a non-simple edge (namely, $j=n$ ), cannot be confined to one side of $H^{\prime}$. Finally, if $W$ is of type $A$, then we know that $\widetilde{\mathcal{H}}$ includes all hyperplanes of the form $\left\{\mu:\left\langle\mu, \beta_{1}+\beta_{2}\right\rangle=1\right\}$ with $\beta_{1}, \beta_{2} \in \Phi$ orthogonal (see the proof of Lemma 3.4). In particular, if $s_{j}$ and $s_{0}$ are not adjacent, then we may take $\beta_{1}=\widetilde{\alpha}$ and $\beta_{2}=\alpha_{j}$ and follow the same reasoning as above to reach a contradiction. 


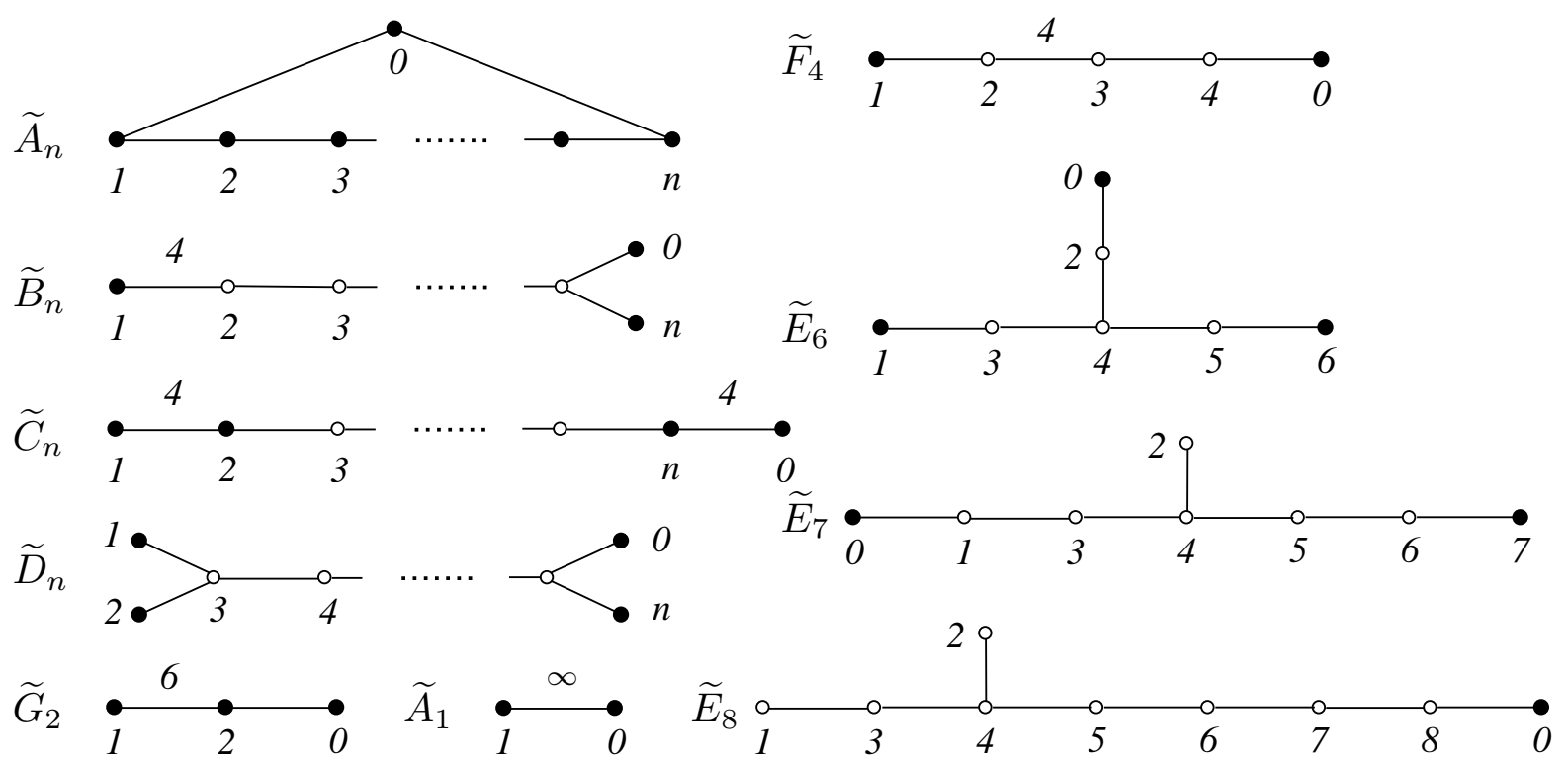

Figure 4: The tight double quotients $\left(\left(\widetilde{W} \cdot \theta_{j}\right)_{S},<_{\mathrm{B}}\right) ; \bullet=$ tight.

\section{The classification.}

We use two stages to classify those $\theta \in A_{0}$ such that $\left((\widetilde{W} \cdot \theta)_{S},<_{\mathrm{B}}\right)$ is tight. In the first stage, we use the following lemma and the results of Section 3 to assist in the analysis of cases involving the vertices $\theta_{j}$ of $A_{0}$. This allows us to deduce a general characterization of tightness analogous to Lemma 3.3, and we use this to complete the classification.

Lemma 5.9. If $\theta \in A_{0}$ and the $W_{\langle i\rangle}$-orbits in the $W$-orbit of $\theta+\mathbf{Z} \Phi^{\vee}$ are stratified, then the $W_{\langle i\rangle}$-orbits in $W \theta$ are also stratified.

Proof. Consider a pair $\mu, \nu \in W \theta$ with $\mu-\nu \in \operatorname{Span} \Phi_{\langle i\rangle}$. Without loss of generality, we may assume that $\mu$ and $\nu$ are the $\Phi_{\langle i\rangle}$-dominant members of their $W_{\langle i\rangle}$-orbits. Given that the $W_{\langle i\rangle}$-orbits in the $W$-orbit of $\theta+\mathbf{Z} \Phi^{\vee}$ are stratified, there is some $w \in W_{\langle i\rangle}$ such that $w \mu-\nu \in \mathbf{Z} \Phi^{\vee}$. However, we also have $x \mu-\nu=(x \mu-\mu)+(\mu-\nu) \in \operatorname{Span} \Phi_{\langle i\rangle}$ for all $x \in W_{\langle i\rangle}$, and hence

$$
w \mu-\nu \in \mathbf{Z} \Phi^{\vee} \cap \operatorname{Span} \Phi_{\langle i\rangle}=\mathbf{Z} \Phi_{\langle i\rangle}^{\vee}
$$

In other words, there is an orbit of the affine Weyl group associated to $\Phi_{\langle i\rangle}$ that includes both $\mu$ and $\nu$. Furthermore, since $\mu$ is $\Phi_{\langle i\rangle}$-dominant and in the $W$-orbit of $A_{0}$, we have $0 \leqslant\langle\mu, \beta\rangle \leqslant 1$ for all $\beta \in \Phi_{\langle i\rangle}^{+}$(and similarly $\nu$ ), so $\mu$ and $\nu$ are both in the fundamental alcove relative to $\Phi_{\langle i\rangle}$, whence $\mu=\nu$ and the orbits stratify.

As a corollary of Proposition 5.2(b), Corollary 3.10 and the above result, one sees that if $\left((\widetilde{W} . \theta)_{S},<_{\mathrm{B}}\right)$ is tight, then $\left(W \theta,<_{\mathrm{B}}\right)$ is tight. On the other hand, the following result shows that the converse fails; e.g., $\left(B_{n} \theta_{2},<_{\mathrm{B}}\right)$ is tight, but $\left(\left(\widetilde{B}_{n} \cdot \theta_{2}\right)_{S},<_{\mathrm{B}}\right)$ is not. 
TheOREM 5.10. If $\widetilde{W}$ is irreducible and $j$ is fixed, the following are equivalent.

(a) The Bruhat ordering of $\left(\left(\widetilde{W} \cdot \theta_{j}\right)_{S},<_{\mathrm{B}}\right)$ is tight.

(b) The $W$-orbit of $\theta_{j}+\mathbf{Z} \Phi^{\vee}$ has stratified $W_{I}$-orbits for all $I \subset S$.

(c) The $W$-orbit of $\theta_{j}+\mathbf{Z} \Phi^{\vee}$ has stratified $W_{\langle i\rangle}$-orbits for all $i$.

(d) Either $j=0$, or one of the following holds:

- $W \cong A_{n}$ or $W \cong G_{2}$,

- $W \cong B_{n}$ and $j=1$ or $n$,

- $W \cong C_{n}$ or $D_{n}$ and $j=1,2$ or $n$,

- $(W, j) \cong\left(E_{6}, 1\right),\left(E_{6}, 6\right),\left(E_{7}, 7\right)$, or $\left(F_{4}, 1\right)$.

The numbering of the vertices of $A_{0}$ in each case is indicated in Figure 4.

Proof. (a) $\Rightarrow(\mathrm{b})$ is Proposition 5.2(a) and (b) $\Rightarrow(\mathrm{c})$ is immediate.

$\neg(\mathrm{d}) \Rightarrow \neg(\mathrm{c})$. If $\left(W \theta_{j},<_{\mathrm{B}}\right)$ is not tight, then there must be some $i$ such that the $W_{\langle i\rangle}$-orbits in $W \theta_{j}$ are not stratified (Theorem 3.9), and hence the $W_{\langle i\rangle}$-orbits in the $W$-orbit of $\theta_{j}+\mathbf{Z} \Phi^{\vee}$ cannot be stratified (Lemma 5.9). By the classification of tight $W$-orbits (Theorem 3.8), this reasoning applies to all of the instances not listed in (d), with two exceptions: $(W, j) \cong\left(B_{n}, 2\right)(n \geqslant 3)$ and $(W, j) \cong\left(F_{4}, 4\right)$.

In the case $W=B_{n}$, the coefficient of $\alpha_{2}$ in $\widetilde{\alpha}$ is 2 (assuming $n \geqslant 3$ ), so if we take $\beta=\alpha_{1}+\alpha_{2} \in \Phi$, then $\left\langle\theta_{2}, \beta\right\rangle=1 / 2$ and $s_{\beta} \theta_{2}=\theta_{2}-(1 / 2) \beta^{\vee}$. Hence,

$$
\theta_{2}-s_{\beta} \theta_{2}=(1 / 2) \alpha_{1}^{\vee}+\alpha_{2}^{\vee} \in \operatorname{Span} \Phi_{\langle 2\rangle}+\mathbf{Z} \Phi^{\vee}
$$

On the other hand, the $W_{\langle 2\rangle}$-orbit of $\theta_{2}$ (and hence also $\theta_{2}+\mathbf{Z} \Phi^{\vee}$ ) is trivial, so $s_{\beta} \theta_{2}+\mathbf{Z} \Phi^{\vee}$ and $\theta_{2}+\mathbf{Z} \Phi^{\vee}$ are in distinct $W_{\langle 2\rangle}$-orbits.

In the case $W=F_{4}$, we have $\theta_{4}=(1 / 2) \alpha_{1}^{\vee}+\alpha_{2}^{\vee}+(3 / 2) \alpha_{3}^{\vee}+\alpha_{4}^{\vee}=(1 / 2) \widetilde{\alpha}^{\vee}$. It follows that the $W$-orbit of $\theta_{4}$ includes $\mu:=(1 / 2) \alpha_{3}^{\vee}$ (and more generally, all $(1 / 2) \beta^{\vee}$ such that $\beta \in \Phi$ is long). Furthermore,

$$
\theta_{4}-\mu=(1 / 2) \alpha_{1}^{\vee}+\alpha_{2}^{\vee}+\alpha_{3}^{\vee}+\alpha_{4}^{\vee} \in(1 / 2) \alpha_{1}^{\vee}+\mathbf{Z} \Phi^{\vee} \subset \operatorname{Span} \Phi_{\langle 4\rangle}+\mathbf{Z} \Phi^{\vee}
$$

However $W_{\langle 4\rangle}$ fixes $\theta_{4}$, so $\mu+\mathbf{Z} \Phi^{\vee}$ and $\theta_{4}+\mathbf{Z} \Phi^{\vee}$ are in distinct $W_{\langle 4\rangle}$-orbits.

$(\mathrm{d}) \Rightarrow(\mathrm{a})$. The only cases listed in (d) not covered by either Theorem 4.10 or Corollary 5.6 are those involving $W=G_{2}$ (with $\left.j \neq 0\right)$. To prove that $\left(\left(\widetilde{G}_{2} \cdot \theta\right)_{S},<_{\mathrm{B}}\right)$ is tight in these cases, it suffices by induction to show that for all dominant $\mu, \nu \in \widetilde{G}_{2} . \theta$ with $\mu \prec \nu$, there is a dominant $\lambda \in \widetilde{G}_{2} . \theta$ such that $\mu \preccurlyeq \lambda<_{\mathrm{B}} \nu$ or $\mu<_{\mathrm{B}} \lambda \preccurlyeq \nu$.

The case $\theta=\theta_{1}$. From the extended diagram, one sees that the stabilizer of $\theta_{1}+\mathbf{Z} \Phi^{\vee}$ is isomorphic to $A_{2}$ and thus has index 2 in $G_{2}$. On the other hand, the coefficient of $\alpha_{1}$ in $\widetilde{\alpha}$ is 3 , so we have $\left\langle\theta_{1}, \alpha_{1}\right\rangle=1 / 3$ and $s_{1} \theta_{1}=\theta_{1}-(1 / 3) \alpha_{1}^{\vee}$, whence

$$
\widetilde{G}_{2} \cdot \theta_{1}=\left(\theta_{1}+\mathbf{Z} \Phi^{\vee}\right) \dot{U}\left(\theta_{1}-\frac{1}{3} \alpha_{1}^{\vee}+\mathbf{Z} \Phi^{\vee}\right)
$$

It follows that $\left\langle\nu, \alpha_{1}\right\rangle \in \mathbf{Z} \pm 1 / 3$ and $\left\langle\nu, \alpha_{2}\right\rangle \in \mathbf{Z}$ for all $\nu \in \widetilde{G}_{2} . \theta_{1}$. 
Given a dominant pair $\mu, \nu \in \widetilde{G}_{2} . \theta_{1}$ such that $\mu \prec \nu$, set $n_{i}=\left\langle\nu, \alpha_{i}\right\rangle(i=1,2)$ and $\nu-\mu=a_{1} \alpha_{1}^{\vee}+a_{2} \alpha_{2}^{\vee}$. It follows via the above considerations that $n_{1} \in\{1 / 3,2 / 3\}+\mathbf{Z}^{\geqslant 0}$, $a_{1} \in(\mathbf{Z} / 3) \geqslant 0$, and $n_{2}, a_{2} \in \mathbf{Z} \geqslant 0$.

If it happens that $a_{1} \in \mathbf{Z}$, then we are done, since Lemmas 4.5 and 4.11 imply that there is a root $\beta$ such that $\mu+\beta^{\vee}$ is dominant and $\mu<_{\mathrm{B}} \mu+\beta^{\vee} \preccurlyeq \nu$.

If $a_{1} \in \mathbf{Z}+2 / 3$, then $n_{1} \in \mathbf{Z}+2 / 3$ is forced; otherwise, we would have $\left\langle\mu, \alpha_{1}\right\rangle \in \mathbf{Z}$ and $\mu \notin \widetilde{G}_{2} \cdot \theta_{1}$. Hence, $\nu-(2 / 3) \alpha_{1}^{\vee}$ is dominant and $\mu \preccurlyeq \nu-(2 / 3) \alpha_{1}^{\vee}<_{\mathrm{B}} \nu$ by (4.1a) unless $\left\langle\nu-(2 / 3) \alpha_{1}^{\vee}, \alpha_{1}\right\rangle=n_{1}-4 / 3<0$; i.e., $n_{1}=2 / 3$. In that case, we must have $a_{2}>0$; otherwise, $a_{2}=0$ and $\left\langle\mu, \alpha_{1}\right\rangle=\left\langle\nu-a_{1} \alpha_{1}^{\vee}, \alpha_{1}\right\rangle=(2 / 3)-2 a_{1}<0$, contradicting the fact that $\mu$ is dominant. Choosing $\beta=2 \alpha_{1}+\alpha_{2} \in \Phi$, we then have $\langle\nu, \beta\rangle=n_{2}+4 / 3 \in \mathbf{Z}+1 / 3$ and $\nu-(1 / 3) \beta^{\vee}=\nu-(2 / 3) \alpha_{1}^{\vee}-\alpha_{2}^{\vee} \succcurlyeq \mu$. Furthermore, $\left\langle\nu-(1 / 3) \beta^{\vee}, \alpha_{i}\right\rangle=1 / 3, n_{2}$ $(i=1,2)$, so $\nu-(1 / 3) \beta^{\vee}$ is dominant and $<_{\mathrm{B}} \nu$, by $(4.1 \mathrm{a})$.

The remaining possibility is $a_{1} \in \mathbf{Z}+1 / 3$, in which case $n_{1} \in \mathbf{Z}+1 / 3$; otherwise, $\left\langle\mu, \alpha_{1}\right\rangle \in \mathbf{Z}$ and $\mu \notin \widetilde{G}_{2} . \theta_{1}$. Hence $\nu-(1 / 3) \alpha_{1}^{\vee}$ is dominant and $\mu \preccurlyeq \nu-(1 / 3) \alpha_{1}^{\vee}<_{\mathrm{B}} \nu$ by (4.1a) unless $\left\langle\nu-(1 / 3) \alpha_{1}^{\vee}, \alpha_{1}\right\rangle=n_{1}-2 / 3<0$; i.e., $n_{1}=1 / 3$. In that case, we must have $a_{2}>0$; otherwise, $\left\langle\mu, \alpha_{1}\right\rangle=\left\langle\nu-a_{1} \alpha_{1}^{\vee}, \alpha_{1}\right\rangle=(1 / 3)-2 a_{1}<0$, contradicting the fact that $\mu$ is dominant. Choosing $\beta=\alpha_{1}+\alpha_{2} \in \Phi$, we then have $\langle\nu, \beta\rangle=n_{2}+1 / 3 \in \mathbf{Z}+1 / 3$ and $\nu-(1 / 3) \beta^{\vee}=\nu-(1 / 3) \alpha_{1}^{\vee}-\alpha_{2}^{\vee} \succcurlyeq \mu$. Furthermore, $\left\langle\nu-(1 / 3) \beta^{\vee}, \alpha_{i}\right\rangle=2 / 3, n_{2}-1$ $(i=1,2)$, so $\nu-(1 / 3) \beta^{\vee}$ is dominant and $<_{\mathrm{B}} \nu$ by (4.1a) unless $n_{1}=1 / 3$ and $n_{2}=0$, in which case $\nu=\theta_{1}$, the minimum element of $\left(\widetilde{G}_{2} \cdot \theta_{1}\right)_{S}$ with respect to $<_{\mathrm{B}}$ (and hence also $\prec)$, a contradiction.

The case $\theta=\theta_{2}$. The stabilizer of $\theta_{2}+\mathbf{Z} \Phi^{\vee}$ is isomorphic to $A_{1} \times A_{1}$ and thus has index 3 in $G_{2}$. On the other hand, the coefficient of $\alpha_{2}$ in $\widetilde{\alpha}$ is 2 , so we have $\left\langle\theta_{2}, \alpha_{2}\right\rangle=1 / 2, s_{2} \theta_{2}=\theta_{2}-(1 / 2) \alpha_{2}^{\vee}$, and $s_{1} s_{2} \theta_{2}=\theta_{2}-(1 / 2) \alpha_{1}^{\vee}-(1 / 2) \alpha_{2}^{\vee}$, whence

$$
\widetilde{G}_{2} \cdot \theta_{2}=\left(\theta_{2}+\mathbf{Z} \Phi^{\vee}\right) \dot{\cup}\left(\theta_{2}-\frac{1}{2} \alpha_{2}^{\vee}+\mathbf{Z} \Phi^{\vee}\right) \dot{\cup}\left(\theta_{2}-\frac{1}{2} \alpha_{1}^{\vee}-\frac{1}{2} \alpha_{2}^{\vee}+\mathbf{Z} \Phi^{\vee}\right) .
$$

It follows that $\left\langle\nu, \alpha_{1}\right\rangle \in \mathbf{Z}+1 / 2$ or $\left\langle\nu, \alpha_{2}\right\rangle \in \mathbf{Z}+1 / 2$ for all $\nu \in \widetilde{G}_{2} \cdot \theta_{2}$.

Given a dominant pair $\mu, \nu \in \widetilde{G}_{2} . \theta_{2}$ such that $\mu \prec \nu$, set $n_{i}=\left\langle\nu, \alpha_{i}\right\rangle(i=1,2)$ and $\nu-\mu=a_{1} \alpha_{1}^{\vee}+a_{2} \alpha_{2}^{\vee}$. The above considerations imply $n_{1}, n_{2}, a_{1}, a_{2} \in(\mathbf{Z} / 2) \geqslant 0$. If it happens that $a_{1}$ and $a_{2}$ are both integers, then we are done, since Lemmas 4.5 and 4.11 imply that there is a root $\beta$ such that $\mu+\beta^{\vee}$ is dominant and $\mu<_{\mathrm{B}} \mu+\beta^{\vee} \preccurlyeq \nu$. Also, it cannot be the case that $\left\langle\mu, \alpha_{1}\right\rangle$ and $\left\langle\mu, \alpha_{2}\right\rangle$ are both integers; otherwise, $\mu$ could not be in the $\widetilde{G}_{2}$-orbit of $\theta_{2}$. This leaves us with three possible parity combinations:

(i) $n_{1}, n_{2}, a_{1}+a_{2} \in \mathbf{Z}+1 / 2$, or

(ii) $n_{1} \in \mathbf{Z}, n_{2}, a_{2} \in \mathbf{Z}+1 / 2$, or

(iii) $n_{2} \in \mathbf{Z}, n_{1}, a_{1} \in \mathbf{Z}+1 / 2$.

In any of these cases, if it happens that $n_{1} \in \mathbf{Z}+1 / 2$ and $a_{1}>0$, then $\nu-(1 / 2) \alpha_{1}^{\vee}$ is dominant and $\mu \preccurlyeq \nu-(1 / 2) \alpha_{1}^{\vee}<_{\mathrm{B}} \nu$ by (4.1a), unless $\left\langle\nu-(1 / 2) \alpha_{1}^{\vee}, \alpha_{1}\right\rangle=n_{1}-1<0$; i.e., $n_{1}=1 / 2$. Also, given that $n_{1}=1 / 2$, we cannot have $a_{2}=0$; otherwise, $a_{1} \geqslant 1 / 2$ and $\left\langle\mu, \alpha_{1}\right\rangle=\left\langle\nu-a_{1} \alpha_{1}^{\vee}, \alpha_{1}\right\rangle=(1 / 2)-2 a_{1}<0$, contradicting the fact that $\mu$ is dominant.

Similar reasoning applies when $n_{2} \in \mathbf{Z}+1 / 2$ and $a_{2}>0$. 
We cannot have $a_{1}=a_{2}=0$, so at least one of the above arguments applies in case (i). However, either argument finishes the induction unless $a_{1}$ and $a_{2}$ are both positive, in which case both arguments apply, leaving only the possibility that $n_{1}=n_{2}=1 / 2$ and $a_{1}, a_{2} \geqslant 1 / 2$. We must also have $a_{2} \geqslant 1$; otherwise, $a_{2}=1 / 2, a_{1} \geqslant 1$, and $\left\langle\mu, \alpha_{1}\right\rangle=$ $\left\langle\nu-(\nu-\mu), \alpha_{1}\right\rangle=(1 / 2)-2 a_{1}+a_{2}<0$, a contradiction. Choosing $\beta=3 \alpha_{1}+2 \alpha_{2} \in \Phi$, we obtain $\langle\nu, \beta\rangle=5 / 2 \in \mathbf{Z}+1 / 2$ and $\nu-(1 / 2) \beta^{\vee}=\nu-(1 / 2) \alpha_{1}^{\vee}-\alpha_{2}^{\vee} \succcurlyeq \mu$. Also, $\left\langle\nu-(1 / 2) \beta^{\vee}, \alpha_{i}\right\rangle=1 / 2,0(i=1,2)$, so $\nu-(1 / 2) \beta^{\vee}$ is dominant and $<_{\mathrm{B}} \nu$, by (4.1a).

The remaining possibilities are $n_{2}=1 / 2, a_{1}>0$ in case (ii) and $n_{1}=1 / 2, a_{2}>0$ in case (iii). In these cases, choose $\beta=3 \alpha_{1}+\alpha_{2} \in \Phi$, so that $\langle\nu, \beta\rangle=3 n_{1}+n_{2} \in \mathbf{Z}+1 / 2$ and $\nu-(1 / 2) \beta^{\vee}=\nu-(1 / 2)\left(\alpha_{1}^{\vee}+\alpha_{2}^{\vee}\right) \succcurlyeq \mu$. We find $\left\langle\nu-(1 / 2) \beta^{\vee}, \alpha_{i}\right\rangle=n_{1}-1 / 2, n_{2}+1 / 2$ $(i=1,2)$, so $\nu-(1 / 2) \beta^{\vee}$ is dominant and $<_{\mathrm{B}} \nu$ unless $n_{1}=0$ and $n_{2}=1 / 2$, in which case $\nu=\theta_{2}$ is the minimum element of $\left(\widetilde{G}_{2} \cdot \theta_{2}\right)_{S}$, a contradiction.

The following is an affine version of Lemma 3.3.

THEOREM 5.11. If $\theta \in A_{0}$ has stabilizer $\widetilde{W}_{J}$, then $\left((\widetilde{W} \cdot \theta)_{S},<_{\mathrm{B}}\right)$ is tight if and only if

(i) $\left(\left(\widetilde{W} . \theta_{j}\right)_{S},<_{\mathrm{B}}\right)$ is tight for all $s_{j} \in \widetilde{S}-J$, and

(ii) there is a face of $\widetilde{\mathcal{H}}$ that contains every $\theta^{\prime} \in A_{0}$ with stabilizer $\widetilde{W}_{J}$.

Proof. If $\left((\widetilde{W} . \theta)_{S},<_{\mathrm{B}}\right)$ is tight, then Lemma 5.7 implies (ii), and Proposition 5.2(b) implies that for all $s_{j} \in \widetilde{S}-J$ and all $i$, the $W$-orbit of $\theta_{j}+\mathbf{Z} \Phi^{\vee}$ has stratified $W_{\langle i\rangle}$-orbits. Hence $\left(\left(\widetilde{W} \cdot \theta_{j}\right)_{S},<_{\mathrm{B}}\right)$ is tight by Theorem 5.10 .

Conversely, suppose we have a relation $x . \theta \preccurlyeq y . \theta$ for some $x, y \in{ }^{S} \widetilde{W}^{J}$. We seek to prove that $x \leqslant_{\mathrm{B}} y$, or equivalently (Corollary 1.4), $x \cdot \theta_{j} \leqslant_{\mathrm{B}} y \cdot \theta_{j}$ for all $j$ such that $s_{j} \in \widetilde{S}-J$. Given that $\left(\left(\widetilde{W} \cdot \theta_{j}\right)_{S},<_{\mathrm{B}}\right)$ is tight, this is equivalent to having $x . \theta_{j} \preccurlyeq y \cdot \theta_{j}$ for all such $j$.

Toward a contradiction, suppose that $x . \theta_{j} \npreceq y . \theta_{j}$ for some $j$. In that case, we have $\left\langle x . \theta_{j}-y . \theta_{j}, \omega_{i}\right\rangle>0$ and $\left\langle x . \theta-y . \theta, \omega_{i}\right\rangle \leqslant 0$ for some $i$. It follows that there are points $\theta^{\prime}$ on the line segment connecting $\theta$ to $\theta_{j}$ near $\theta$ (and thus having stabilizer $\widetilde{W}_{J}$ ) that lie on and off the hyperplane

$$
H=\left\{\mu \in V:\left\langle x \cdot \mu-y \cdot \mu, \omega_{i}\right\rangle=0\right\} .
$$

Letting $x_{0} \in W$ denote the linear part of $x$ (so that $x=t_{\mu} x_{0}$ for some $\mu \in \mathbf{Z} \Phi^{\vee}$ ) and similarly $y_{0}$, it follows that $x . \mu-y \cdot \mu$ differs from $x_{0} \mu-y_{0} \mu$ by a member of $\mathbf{Z} \Phi^{\vee}$, and thus there is an integer $k$ such that $H=H\left(x_{0}^{-1}, y_{0}^{-1}, i, k\right) \in \widetilde{\mathcal{H}}$, contradicting (ii).

The above result shows that if $\theta$ has stabilizer $\widetilde{W}_{J}$, then the tightness of $\left((\widetilde{W} . \theta)_{S},<_{\mathrm{B}}\right)$ depends only on $J$, not the choice of $\theta$. We remark that this independence may also be proved directly. Indeed, it is not hard to show that if $F$ is a face of $A_{0}$ that is confined to a single face of $\widetilde{\mathcal{H}}$, then $\left((\widetilde{W} \cdot \theta)_{S}, \prec\right)$ is independent of $\theta \in F$. 
THEOREM 5.12. If $\widetilde{W}$ is irreducible, then $\left((\widetilde{W} \cdot \theta)_{S},<_{\mathrm{B}}\right)$ is tight for some (equivalently, all) $\theta \in A_{0}$ with stabilizer $\widetilde{W}_{J}$ if and only if $J^{c}=\left\{s_{0}\right\}$ or one of the following holds:

- $W \cong A_{n}$ and $J^{c}=\left\{s_{j}\right\},\left\{s_{j}, s_{j+1}\right\}(0 \leqslant j \leqslant n)$, or ( $n=2$ only) $\left\{s_{0}, s_{1}, s_{2}\right\}$,

- $W \cong B_{n}$ and $J^{c}=\left\{s_{1}\right\}$, or $\left\{s_{n}\right\}$,

- $W \cong C_{n}$ and $J^{c}=\left\{s_{1}\right\},\left\{s_{2}\right\},\left\{s_{n}\right\},\left\{s_{1}, s_{2}\right\}$, or $\left\{s_{0}, s_{n}\right\}$,

- $W \cong D_{n}$ and $J^{c}=\left\{s_{1}\right\},\left\{s_{2}\right\}$, or $\left\{s_{n}\right\}$,

- $W \cong E_{6}$ and $J^{c}=\left\{s_{1}\right\}$ or $\left\{s_{6}\right\}$,

- $W \cong E_{7}$ and $J^{c}=\left\{s_{7}\right\}$,

- $W \cong F_{4}$ and $J^{c}=\left\{s_{1}\right\}$, or

- $W \cong G_{2}$ and $J^{c}=\left\{s_{1}\right\},\left\{s_{2}\right\}$, or $\left\{s_{1}, s_{2}\right\}$.

The numbering of the simple reflections in each case is indicated in Figure 4, with the added convention that in the case $W \cong A_{n}$, we identify $s_{n+1}$ with $s_{0}$.

Proof. For the cases in which $J^{c}=\left\{s_{j}\right\}$ is a singleton (equivalently, $\theta=\theta_{j}$ ), the above list agrees with Theorem 5.10. In the remaining cases, in order for $\left((\widetilde{W} . \theta)_{S},<_{\mathrm{B}}\right)$ to be tight, Lemmas 5.7 and 5.8 show that $J^{c}$ must be comprised of subsets from the list in Theorem 5.10 that are mutually adjacent in the diagram of $\widetilde{W}$, allowing simple adjacencies only if $W$ is of type $A$. This leaves the following possibilities:

- $W=A_{n}$ and $J^{c}=\left\{s_{j}, s_{j+1}\right\}(0 \leqslant j \leqslant n)$,

- $W=A_{2}$ and $J^{c}=\widetilde{S}=\left\{s_{0}, s_{1}, s_{2}\right\}$,

- $W=C_{n}$ and $J^{c}=\left\{s_{0}, s_{n}\right\}$ or $J^{c}=\left\{s_{1}, s_{2}\right\}$, and

- $W=G_{2}$ and $J^{c}=\left\{s_{1}, s_{2}\right\}$.

To complete the proof, it suffices by Theorem 5.11 to show that in each of these cases, the face $F_{J}$ of $A_{0}$ with stabilizer $\widetilde{W}_{J}$ is confined to a single face of $\widetilde{\mathcal{H}}$.

In the standard coordinates for $W=A_{n}$, we have $\alpha_{i}=\varepsilon_{i}-\varepsilon_{i-1}(1 \leqslant i \leqslant n)$, where $\varepsilon_{0}, \varepsilon_{1}, \ldots, \varepsilon_{n}$ are orthonormal. Furthermore, $\widetilde{\mathcal{H}}$ consists of all hyperplanes of the form $\{\mu:\langle\mu, \gamma\rangle=k\}$, where $k \in \mathbf{Z}$ and $\gamma=\sum a_{i} \varepsilon_{i}$ ranges over vectors with $\sum a_{i}=0$ and $a_{i} \in\{0, \pm 1\}$. If $J^{c}=\left\{s_{j}, s_{j+1}\right\}$, the face $F_{J}$ consists of all $\theta=a \varepsilon+b \varepsilon_{j}+\left(\varepsilon_{j+1} \cdots+\varepsilon_{n}\right)$, where $\varepsilon=\left(\varepsilon_{0}+\cdots+\varepsilon_{n}\right)$ and $0<b<1$ ( $a$ is arbitrary). It follows that the evaluation of $\theta$ against each normal vector $\gamma$ has the form $m \pm b$ or $m$ for various integers $m$, and thus $\theta$ is confined to a single face of $\widetilde{\mathcal{H}}$. Note also that if $n=2$, the normal vectors $\gamma$ are precisely the roots, and thus $F_{\varnothing}=A_{0}$ is by definition one of the faces of $\widetilde{\mathcal{H}}$.

In the case $W=C_{n}$, we have $\alpha_{1}=2 \varepsilon_{1}$ and $\alpha_{i}=\varepsilon_{i}-\varepsilon_{i-1}(1<i \leqslant n)$, and $\widetilde{\mathcal{H}}$ consists of all hyperplanes of the form $\{\mu:\langle\mu, \gamma\rangle=k\}$, where $k \in \mathbf{Z}$ and $\gamma=\sum a_{i} \varepsilon_{i}$ ranges over vectors with $\sum a_{i}$ even and $a_{i} \in\{0, \pm 1, \pm 2\}$. Given $J^{c}=\left\{s_{0}, s_{n}\right\}$, the face $F_{J}$ consists of all $\theta=(a / 2) \varepsilon_{n}$ with $0<a<1$. Thus the evaluation of $\theta$ against each normal vector $\gamma$ has the form $0, \pm a / 2$, or $\pm a$. Similarly, if $J^{c}=\left\{s_{1}, s_{2}\right\}$, then $F_{J}$ consists of all $\theta=(a / 2) \varepsilon_{1}+(1 / 2)\left(\varepsilon_{2}+\cdots+\varepsilon_{n}\right)$ with $0<a<1$. In this case, the evaluations of $\theta$ against the normal vectors all have the form $m, m \pm a$, or $m+(1 / 2) \pm(a / 2)$ for various integers $m$. Thus in both cases, $\theta$ is confined to a single face of $\widetilde{\mathcal{H}}$.

In the case $W=G_{2}$, the fundamental weights are roots, so $\widetilde{\mathcal{H}}$ consists of all hyperplanes of the form $\{\mu:\langle\mu, \gamma\rangle=k\}$, where $k \in \mathbf{Z}$ and $\gamma$ ranges over all (nonzero) sums 
of pairs of roots that are both short or both long. It is not hard to show that this forces $\gamma$ to be either a root, twice a root, or three times a short root. We may ignore the root hyperplanes, since they define the faces of $A_{0}$. The long roots are $\alpha_{2}, 3 \alpha_{1}+\alpha_{2}$ and $3 \alpha_{1}+2 \alpha_{2}$; if we double their evaluations against $\theta=a \theta_{1}+(1-a) \theta_{2}$, we obtain $1-a$, $1+a$, and 2. Similarly, the short roots are $\alpha_{1}, \alpha_{1}+\alpha_{2}$ and $2 \alpha_{1}+\alpha_{2}$; if we double their evaluations against $\theta$, we obtain $2 a / 3,1-a / 3$, and $1+a / 3$; tripling yields $a, 3 / 2-a / 2$, and $3 / 2+a / 2$. Thus in each case, no integer value is crossed for $0<a<1$, so the face corresponding to $J^{c}=\left\{s_{1}, s_{2}\right\}$ is confined to a single face of $\widetilde{\mathcal{H}}$.

Note that in each case, the subsets $J \subset \widetilde{S}$ appearing in Theorem 5.12 are closed under automorphisms of the diagram of $\widetilde{W}$. Since these automorphisms act transitively on the subsets of minuscule type, Theorem 5.1 yields the following.

COROLlary 5.13. The double quotient $\left({ }^{I} \widetilde{W}^{J},<_{\mathrm{B}}\right)$ has a tight affine embedding if and only if $I$ is of minuscule type and $J$ is listed in Theorem 5.12.

REMARK 5.14. The same reasoning used in the proof of Theorem 4.10 shows that if $\left({ }^{I} \widetilde{W}^{J},<_{\mathrm{B}}\right)$ has a tight affine embedding, then it has order dimension $|S|$. For example, it follows in this way that $\left({ }^{\langle i\rangle} \widetilde{S}_{n}^{J},<_{\mathrm{B}}\right)$ has order dimension $n-1$ for all $i$ and all $J$ such that $J^{c}=\left\{s_{j}, s_{j+1}\right\}$ for some $j$. Furthermore, Theorem 1.3 shows that $\left({ }^{\langle i\rangle} \widetilde{S}_{n},<_{\mathrm{B}}\right)$ is the conjunction of $\lceil n / 2\rceil$ such partial orders, so we deduce that

$$
\begin{aligned}
\operatorname{dim}\left(\widetilde{S}_{n}^{\langle i\rangle},<_{\mathrm{B}}\right) & \leqslant(n-1) \cdot\lceil n / 2\rceil, \\
\operatorname{dim}\left(\widetilde{S}_{n},<_{\mathrm{B}}\right) & \leqslant n(n-1) \cdot\lceil n / 2\rceil .
\end{aligned}
$$

\section{References.}

[BW] A. Björner and M. Wachs, Bruhat order of Coxeter groups and shellability, Adv. in Math. 43 (1982), 87-100.

[B] N. Bourbaki, "Groupes et Algèbres de Lie, Chp. IV-VI," Masson, Paris, 1981.

[D1] V. V. Deodhar, Some characterizations of Bruhat ordering on a Coxeter group and determination of the relative Möbius function, Invent. Math. 39 (1977), 187-198.

[D2] V. V. Deodhar, On Bruhat ordering and weight-lattice ordering for a Weyl group, Nederl. Akad. Wetensch. Indag. Math. 40 (1978), 423-435.

[DG] P. Diaconis and A. Gangolli, Rectangular arrays with fixed margins, in "Discrete probability and algorithms (Minneapolis, MN, 1993)," IMA Vol. Math. Appl. 72, Springer, New York, 1995, pp. 15-41.

[H] J. E. Humphreys, "Reflection Groups and Coxeter Groups," Cambridge Univ. Press, Cambridge, 1990.

[K] S. Kumar, "Kac-Moody groups, their flag varieties and representation theory," Birkhäuser, Boston, 2002. 
[L] G. Lusztig, Singularities, character formulas, and a $q$-analog of weight multiplicities, in "Analysis and topology on singular spaces II-III," Astérisque 101-102 (1983), 208-229.

[MP] R. V. Moody and J. Patera, Fast recursion formula for weight multiplicities, Bull. Amer. Math. Soc. 7 (1982), 237-242.

[P1] R. A. Proctor, Classical Bruhat orders and lexicographic shellability, J. Algebra 77 (1982), 104-126.

[P2] R. A. Proctor, Bruhat lattices, plane partition generating functions, and minuscule representations, Europ. J. Combin. 5 (1984), 331-350.

[R] N. Reading, Order dimension, strong Bruhat order and lattice properties for posets, Order 19 (2002), 73-100.

[RW] N. Reading and D. Waugh, The order dimension of Bruhat order on infinite Coxeter groups, Electron. J. Combin. 11(2) (2005), R13.

[St] R. P. Stanley, Weyl groups, the hard Lefschetz theorem, and the Sperner property, SIAM J. Algebraic Discrete Methods 1 (1980), 168-184.

[S1] J. R. Stembridge, On the fully commutative elements of Coxeter groups, J. Algebraic Combin. 5 (1996), 353-385.

[S2] J. R. Stembridge, The partial order of dominant weights, Advances in Math. 136 (1998), 340-364.

[S3] J. R. Stembridge, Quasi-minuscule quotients and reduced words for reflections, J. Algebraic Combin. 13 (2001), 275-293.

[S4] J. R. Stembridge, A weighted enumeration of maximal chains in the Bruhat order, J. Algebraic Combin. 15 (2002), 291-301. 